\title{
The kinematics in the pc-scale jets of AGN
}

\section{The case of S5 $1803+784^{\star}$}

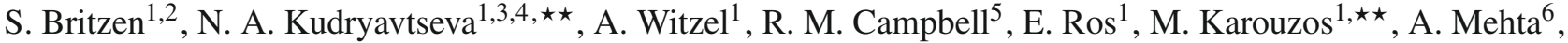 \\ M. F. Aller ${ }^{7}$, H. D. Aller ${ }^{7}$, T. Beckert ${ }^{1}$, and J. A. Zensus ${ }^{1}$ \\ 1 Max-Planck-Institut für Radioastronomie, Auf dem Hügel 69, 53121 Bonn, Germany \\ e-mail: sbritzen@mpifr-bonn.mpg.de \\ 2 Landessternwarte, Königstuhl, 69117 Heidelberg, Germany \\ 3 St. Petersburg State University, Petrodvoretz, St.-Petersburg, Russia \\ ${ }^{4}$ Physics Department, University College Cork, Cork City, Cork, Ireland \\ 5 Joint Institute for VLBI in Europe, Oude Hoogeveensedijk 4, 7991 PD Dwingeloo, The Netherlands \\ ${ }^{6}$ International University Bremen, Postfach 750561, 28725 Bremen, Germany \\ 7 Astronomy Department, University of Michigan, Ann Arbor, MI 48109-1042, USA
}

Received 18 December 2007 / Accepted 23 August 2008

\begin{abstract}
Context. BL Lac objects show core-jet structures with features moving outwards along the jet. We present a kinematic analysis of jet component motion in the pc-scale jet of the BL Lac object S5 1803+784, which does not reveal long-term outward motion for most of the components.

Aims. S5 1803+784 shows complex kinematic phenomena; understanding these provides new insights into the emission processes in BL Lac objects and possibly into the differences between quasars and BL Lac objects.

Methods. The blazar S5 1803+784 has been studied with VLBI at $v=1.6,2.3,5,8.4$, and $15 \mathrm{GHz}$ between 1993.88 and 2005.68 in 26 observing runs. We (re)analyzed the data and present Gaussian model-fits. We collected the already published kinematic information for this source from the literature and re-identified the components according to the new scenario presented in this paper. Altogether, 94 epochs of observations have been investigated.

Results. A careful study of the long-term kinematics reveals a new picture for jet component motion in S5 1803+784. In contrast to previously discussed motion scenarios, we find that the jet structure within 12 mas of the core can most easily be described by the coexistence of several bright jet features that remain on the long-term at roughly constant core separations (in addition to the already known "stationary" jet component $\sim 1.4$ mas) and one faint component moving with an apparent superluminal speed ( $19 c$, based on 3 epochs). While most of the components maintain long-term roughly constant distances from the core, we observe significant, smooth changes in their position angles. We report on an evolution of the whole jet ridge line with time over the almost 12 years of observations. The width of the jet changes periodically with a period of $\sim 8-9$ years. We find a correlation between changes in the position angle and maxima in the total flux-density light-curves. We present evidence for a geometric origin of the observed phenomena and discuss possible models.

Conclusions. We find evidence for a significantly different scenario of jet component motion in S5 1803+784 compared to the generally accepted one of outwardly moving jet components, and conclude that the observed phenomena (evolution of the jet ridge line, roughly constant component core separations but with significant position angle changes) can most easily be explained within a geometric model.
\end{abstract}

Key words. techniques: interferometric - BL Lacertae objects: individual: S5 1803+784 - radio continuum: galaxies BL Lacertae objects: general

\section{Introduction}

Jet component motion in most active galactic nuclei (AGN) has been successfully described by relativistic shock-in-jet motion directed away from the presumed central engine (e.g., Marscher \& Gear 1985). Bulk relativistic motion explains many observed jet-related phenomena and is also important with regard to the observed short-term radio variability (IDV,

* Figures 15 to 20 and Tables 1, 2, 7, and 8 are only available in electronic form at http://www . aanda.org

$\star \star$ Member of the International Max Planck Research School (IMPRS) for Radio and Infrared Astronomy at the Universities of Bonn and Cologne.
Wagner \& Witzel 1995, and references therein) providing the necessary Doppler factors to reduce the observed brightness temperatures to the Compton limit (Kellermann \& Pauliny-Toth 1969). However, as more detailed VLBI-monitoring reveals, this model can not easily explain all the observed motion phenomena in a growing number of AGN. In many sources stationary features or even motion towards the core have been observed (e.g., Alberdi et al. 2000; Kellermann et al. 2004; Britzen et al. 2007).

Taking the core as the easternmost component, the brightest jet feature (in $8.4 \mathrm{GHz}$ observations) at $r=1.4$ mas used to be one of the most prominent candidates for a stationary component (e.g., Eckart et al. 1986; Schalinski et al. 1988). However, as we showed in an earlier work, this so-called stationary component 
has a varying core-separation (Britzen et al. 2005a). Based on a more detailed analysis of new data, we show in this paper that most of the jet components in the pc-scale jet of S5 1803+784 (out to $r \sim 14$ mas) seem to remain at roughly constant core separations on the long term, but their position angles undergo significant change. We present the results of a detailed kinematic analysis of the pc-scale jet in this source and discuss possible explanations of these phenomena.

\subsection{The blazar S5 $1803+784$}

As a member of the complete S5 sample of 13 flat-spectrum radio sources at high declinations (Witzel 1987), S5 1803+784 has been observed repeatedly at many frequencies and angular resolutions since the late 1970s (e.g., Eckart et al. 1986, 1987; Schalinski et al. 1988; Witzel et al. 1988; Krichbaum et al. 1993; Britzen \& Krichbaum 1995). These observations revealed the complex morphology of the milli-arcsecond-jet of this intra-day variable source (Wagner \& Witzel 1995, and references therein). The source shows a pronounced jet with prominent jet components located at core separations of $r=1.4,5$, and 12 mas (e.g., Eckart et al. 1986). Geodetic and astronomical VLBI data gathered at 8.4 and $5 \mathrm{GHz}$ between 1979-1987 showed that the component located at $r=1.4$ mas appears stationary (e.g., Schalinski et al. 1988; Witzel et al. 1988). Several authors confirm this constant core separation, leading to a subluminal velocity of $\beta_{\text {app }}<0.74 h^{-1}$ (e.g., Cawthorne et al. 1993). Britzen et al. (2005a) presented an overview over the pc-scale structure of S5 $1803+784$, including discussion of the kinematics of this stationary $r=1.4$ mas component. Based on a jet-component identification procedure in which the brightest jet component in every epoch is associated with this one, this $r=1.4$ mas component approaches the core whenever a new jet component was or was about to be ejected from the core. Thus this stationary component was found to have non-constant core separation ("oscillatory" type behaviour). Britzen et al. (2005a) explain this motion scenario within a model of reconfinement shocks. In addition, three jet components were reported to move with apparent superluminal speeds of $7-10 c$.

S5 1803+784 shows curved jet structure on all scales probed with VLBI. The curvature found in the cm-regime is described in Britzen et al. (2005b). In the mm-regime, the inner jet components move superluminally with expansion rates of $0.14 \pm$ $0.04 \mathrm{mas} \mathrm{yr}^{-1}$ and $0.07 \pm 0.05 \mathrm{mas} \mathrm{yr}^{-1}$, with possibly variable proper motions (0.02-0.28 mas $\mathrm{yr}^{-1}$ ) along a curved path, suggesting helical motion (e.g., Krichbaum 1990). This source also belongs to the group of misaligned AGN (Antonucci et al. 1986; Appl et al. 1996), with the pc- and kpc-scale jet aligned almost perpendicularly. The probable transition region between the pcand kpc-scale jet has been investigated in world array observations by Britzen et al. (2005b). A summary of the observations of the kpc-scale structure can be found therein.

Velocities computed in the previously cited papers have been determined with differing sets of cosmological parameters. Here, we use the following: $H_{0}=71 \mathrm{~km} \mathrm{~s}^{-1} \mathrm{Mpc}^{-1}, \Omega_{\mathrm{M}}=0.27$, $\Omega_{\Lambda}=0.73$, and take $z=0.68$ (Stickel et al. 1993; Lawrence et al. 1987).

\subsection{New scenario for jet motion in $S 51803+784$}

This is the third paper in a series of papers on the radio structure of S5 $1803+784$ and presents an alternative explanation for the observed kinematics in the pc-scale jet compared to the scenario presented in Britzen et al. (2005a). Preliminary results have already been published in Kudryavtseva et al. (2006).

An obvious difference between the motion scenario presented in this paper compared to that in Britzen et al. (2005a) is the significantly larger time span covered by the observations upon which the present one is based. By combining observations covering more than 20 years (in the case of the $1.6 \mathrm{GHz}$ observations), it is possible to investigate the long-term motion. This long-term behaviour shows no significant outward motion of most of the components in this source.

The most prominent jet component in the pc-scale jet of S5 $1803+784$ at frequencies between 5 and $22 \mathrm{GHz}$ is the 1.4 mas component to the west of the easternmost, brightest component. This $r=1.4$ mas component has repeatedly been characterized as stationary. As densely sampled geodetic observations have shown, this stationary component approaches the core from time to time, showing a sort of oscillatory behavior (Britzen et al. 2005a). In this paper we take note of the fact that since Eckart et al. (1986) other jet components have repeatedly been observed at similar core separations of e.g., 6 and 12 mas (Britzen et al. 2005b, and references therein).

Despite the fact that some jet components have been observed at roughly constant core separations over long periods, they have been identified in the literature with components showing long-term outward motion. We here propose a new component identification assuming that the components appearing repeatedly at the same distance from the core indeed represent the same component. We compare our results with results published in the literature and apply our identification scheme to these data and find that the literature data are fully consistent with our new identification scenario.

Several stationary components have been reported for a number of other AGN in the literature. These components remain at a similar position in core separation and position angle. However, here we encounter jet components that remain at roughly constant core separations but show significant changes in their position angle.

We use multi-frequency data to study the kinematics of the components, present a correlation analysis among a number of properties including the flux-density evolution, propose an alternative identification scenario, and conclude with the resulting implications. The structure of this paper is organized as follows: Sect. 2 overviews the data sets that have been observed and analyzed or re-analyzed. Section 3 presents the results of a detailed model-fitting analysis of the 26 epochs of observation and shows evidence for an alternative identification scenario and unusual jet kinematics in S5 1803+784 based on 94 epochs altogether (data re-analyzed plus literature data). In addition, we compare our results with kinematic information derived from the literature. Section 4 summarizes the kinematic results. Section 5 presents correlations and periodicities from our analysis. Finally, Sect. 6 briefly discusses the implications for our understanding of kinematics and emission processes in AGN.

\section{Observations and data reduction}

We have (re-)analyzed 26 multi-frequency $(v=1.6,2.3,5$, 8.4 , and $15 \mathrm{GHz}$ ) VLBI observations of S5 $1803+784$, performed between 1993.88 and 2001.87 with heterogeneous arrays: VLBI observations at 1.6 and $2.3 \mathrm{GHz}$ conducted by Marcaide et al. (1995a,b, 1997); at $5 \mathrm{GHz}$ by Marcaide et al. (1995a,b, 1997), Guirado et al. (2001), and Gurvits et al. (priv. comm.); at $8.4 \mathrm{GHz}$ by Pérez-Torres et al. (2000), Ros et al. (2000, 2001, in prep.); and at $15 \mathrm{GHz}$ by 
Pérez-Torres et al. (2000, in prep.), Kellermann et al. (1998), Zensus et al. (2002), Lister \& Homan (2005). The data have been fringe-fitted and calibrated before by the individual observers. For calibration details we refer the reader to the original publications.

In addition, we performed space-VLBI observations at 1.6 GHz in 2001.87. These observations were scheduled for the VLBA plus HALCA. Unfortunately we did not obtain any data from ground-space baselines and use this observation as a purely ground-based experiment. The data have been fringefitted within AIPS.

We fit circular Gaussian components to each of the data sets at each frequency using the difmap package (v.2.4b, Shepherd 1997). In order to find the optimum set of components and parameters, we fit every data set starting from a point-like model. We used circular Gaussian components in order to avoid the effects of correlations among weakly-constrained axialratio estimates and those of other parameters of the Gaussian components.

\subsection{Determination of the uncertainties}

The determination of model-fit parameter uncertainties is a complex topic and no satisfying solution to this problem has been presented so far. A detailed discussion of model-fitting uncertainties and their influence on the parameters is presented in Britzen et al. (2007, 2008). The uncertainties of each data set in general are influenced by different $u-v$ coverages, calibrations, sensitivities, etc. The relative positional errors are different at different core separations and the brightness of the individual component affects the fitted position as well. Uncertainties should reflect all these different error sources. In order to obtain reliable error estimates we used two different algorithms to determine the model-fitting uncertainties. In the first, the uncertainties for the core separation and the position angle are given by the formulas $\Delta r=\left(d \sigma_{\text {rms }} \sqrt{1+S_{\text {peak }} / \sigma_{\text {rms }}}\right) / 2 S_{\text {peak }}$ and $\Delta \theta=\arctan (\Delta r / r)$, where $\sigma_{\text {rms }}$ is the residual noise of the map after the subtraction of the model, $d$ is the full width at half maximum (FWHM) of the component and $S_{\text {peak }}$ is the peak flux density (Fomalont 1999). However, this formula tends to underestimate the uncertainty if the peak flux-density is very high or the width of the component is small. In the second, we calculated all uncertainties by comparing different model fits ( \pm 1 component) obtained for the same set of data. These uncertainties reflect the possible parameter ranges for the individual components within model-fitting. To be conservative, we selected the maximum of the uncertainties from the two methods as the value of the uncertainty to use in subsequent analysis.

\subsection{Data table}

In Table 1 we list the parameters of the best fits to the data and their uncertainties. We also give the component identification and the reference to the original publication of the data sets. In Figs. 15-20 we show the hybrid images with modelfit components superimposed. For the images from observations between February 2001 and September 2005, the beam sizes have been adjusted for better comparison. The original beams were: epoch $2002(15 \mathrm{GHz}) 0.4207 \times 0.6068$ mas, epoch $2003(15 \mathrm{GHz}) 0.4764 \times 0.4929$ mas, epoch 2005/2 $(15 \mathrm{GHz}) 0.4768 \times 0.4962$ mas, epoch $2001.09(8 \mathrm{GHz})$ $0.697 \times 1.036$ mas, epoch $2001.29(5 \mathrm{GHz}) 1.137 \times 1.651$ mas.

\subsection{Component identification}

Our kinematic analysis relies critically on the component identification. Our new scenario is based on the flux-density, core separation, and position angle of the individual components. All components have been identified in a way such that all of these parameters vary as little as possible between adjacent epochs. For those components that have been identified at several frequencies, we did not find a significant frequency-dependent offset. The results are presented and discussed in detail in the following sections.

\section{Results}

\subsection{Lack of long-term $r(t)$ change in most of the model jet components}

In addition to the formerly so-called stationary component $(\mathrm{Ca})$ at $\sim 1.4$ mas, we find components at core separations of $\sim 0.3$ mas $(\mathrm{C} 0), \sim 0.8$ mas $(\mathrm{C} 1), \sim 2$ mas $(\mathrm{C} 2), \sim 3-4$ mas $(\mathrm{C} 4)$, $\sim 6-8$ mas (C8), and $\sim 10-12$ mas (C12). Most of these jet components - based on the new data presented here - remain at similar core separations between 1993.88 and 2005.68. The core separations of $\mathrm{C} 12$ and $\mathrm{C} 8$ at $15 \mathrm{GHz}$ are less well defined, reflected by their larger uncertainties. Since the identification of $\mathrm{C} 12$ suffers from a reduced number of data points, we do not include this component in our further analysis. Components C30 and $\mathrm{C} 40$ can only be traced at $1.6 \mathrm{GHz}$, and also will not be considered in the detailed analysis. Figure 1 panels $(\mathrm{a}-\mathrm{d})$ present an overview of the $r(t)$ behavior for all jet components at 15 , $8.4,5$, and $1.6 \mathrm{GHz}$, respectively, out to a core separation of $\sim 50$ mas and over a time span of up to twenty years. Panels (b) and (c) show data collected from the literature, but with component re-identifications following the scenario we present in this paper; these previously published data support this new component identification scenario.

\subsubsection{Comparison with data from the literature}

In order to check whether the components we find showed a similar or different kinematic behavior in earlier times, we collected all available VLBI information for S5 $1803+784$ from the literature. We list the model-fit parameters derived for the total intensity VLBI observations in Table 2 . We re-identified all the components from these published model fits according to the scenario proposed in this paper and plot the results in Fig. 1 panels (b) and (c). At both frequencies $(5,8.4 \mathrm{GHz})$, the components have remained at similar core separations for almost 20 years; we find no long-term outward, apparent superluminal motion based on the aggregate data. Although different kinematic scenarios have been proposed by different authors, the data are fully consistent with a scenario in which the components tend to remain at long-term roughly constant core separations.

Panel (d) of Fig. 1 shows $r(t)$ from the $1.6 \mathrm{GHz}$ data. We again find that the inner components (up to 12 mas) maintain roughly constant core separations, although the outer components (C30 and C40) do show some evidence for outward motion.

In Table 3 we give the parameters of the linear regressions performed for the jet components' core separation as a function of time, $r_{\text {mean }}$ and $\mu_{r}$ and calculated apparent speeds, $\beta_{\text {app }}$. Based on the values for the apparent speeds calculated for all frequencies, we find no superluminal motion for the components within $\sim 4$ mas of the core. $\mathrm{C} 1$ and $\mathrm{C} 2$ show no significant 


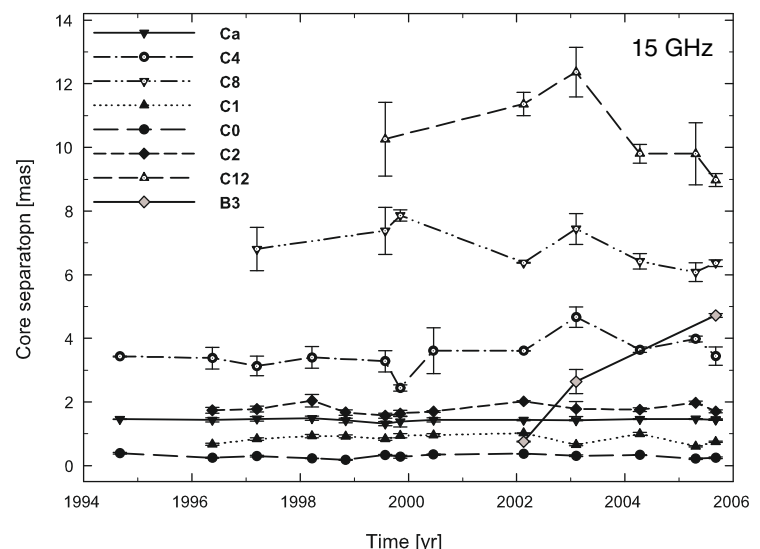

(a)

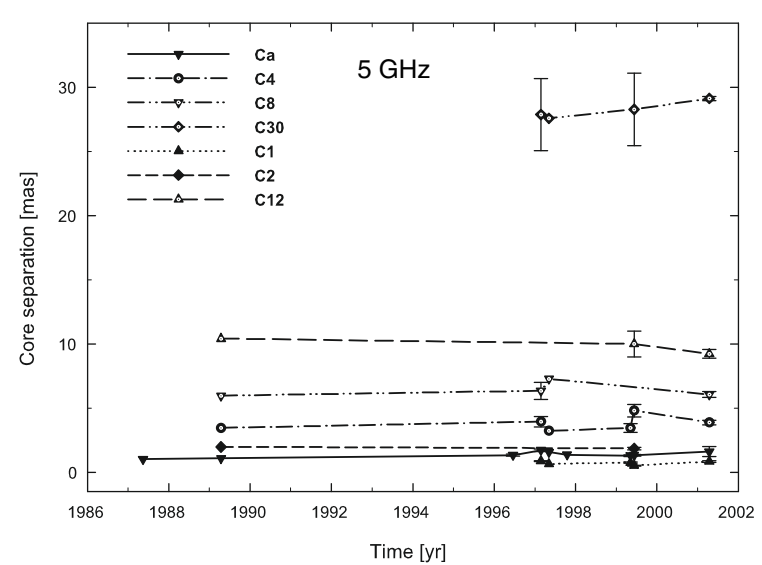

(c)

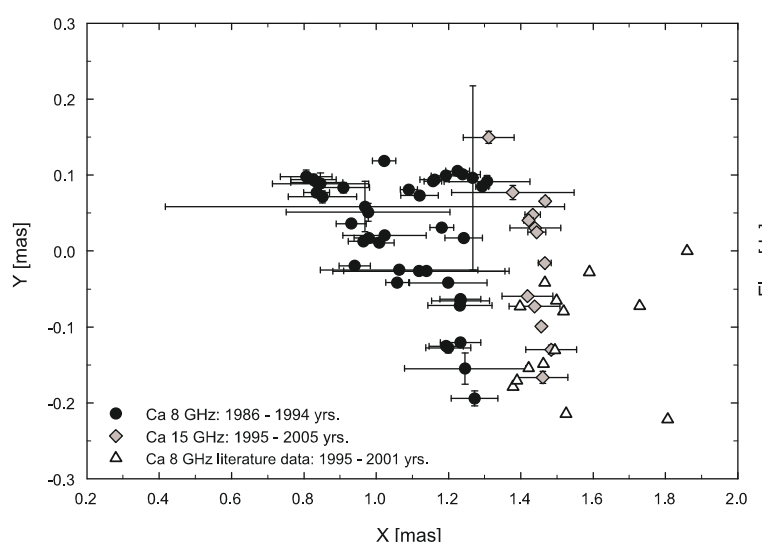

(e)

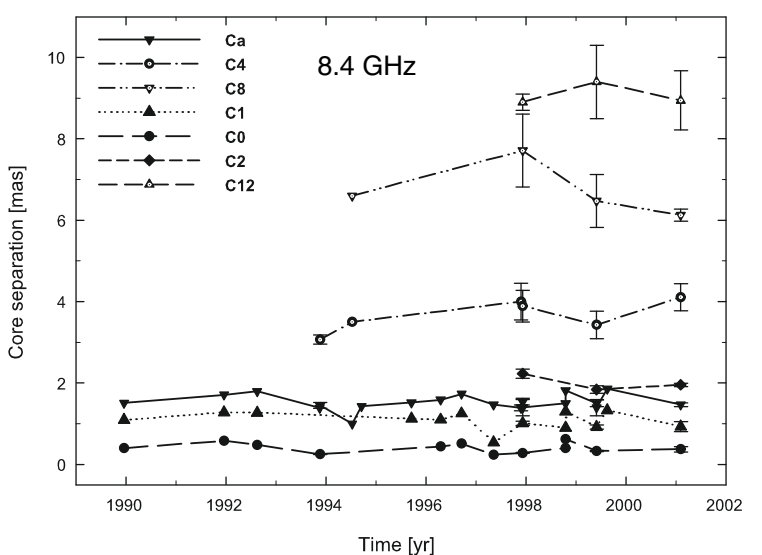

(b)

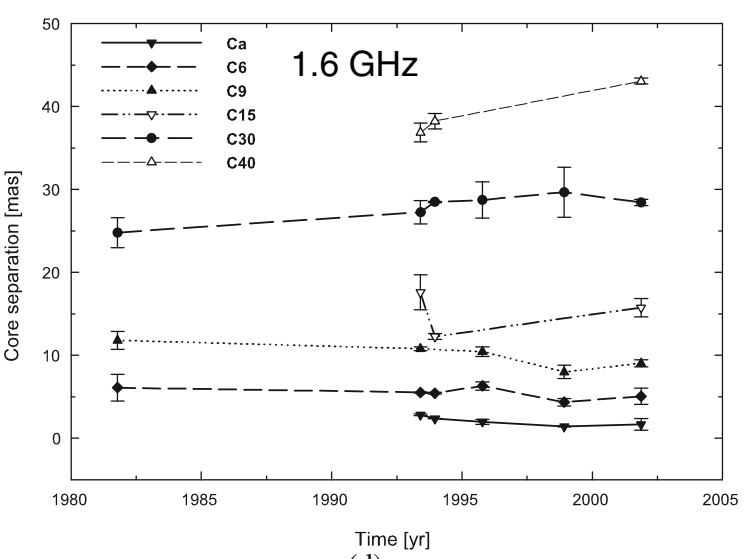

(d)

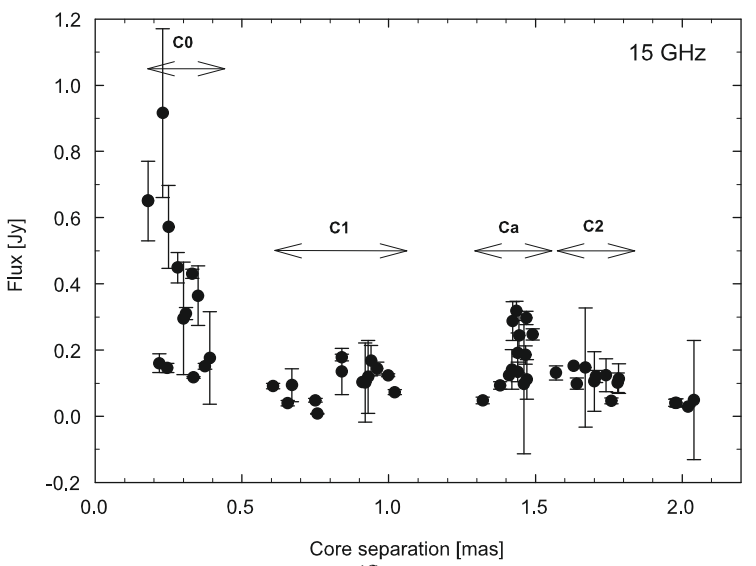

(f)

Fig. 1. Panel a) shows the core separation as function of time, $r(t)$, for those jet components detected at $15 \mathrm{GHz}$. Individual components are denoted by different symbols and lines (usage common across all panels). Note the straight line for component Ca and the fast moving component B3. Panels b) and c) show $r(t)$ for $8.4 \mathrm{GHz}$ and $5 \mathrm{GHz}$ observations, respectively, based on data collected from the literature, with component reidentification following the new scenario discussed in this paper. Panel d) shows $r(t)$ for components farther out along the jet based on $1.6 \mathrm{GHz}$ observations (new + from the literature). Panel e) shows the motion of component $\mathrm{Ca}$ in rectangular coordinates at $8.4 \mathrm{and} 15 \mathrm{GHz}$, in which a displacement towards larger $x$ values is apparent between the fits from the earlier (1986-1994) and newer (1995-2001) $8.4 \mathrm{GHz}$ data with the $15 \mathrm{GHz}$ fits falling in between. Panel f) shows the flux-density along the jet based on all new epochs of the $15 \mathrm{GHz}$ data. The $x$ and $y$ axes scales differ across panels a)-d).

motion within the errors. $\mathrm{C} 0$ and $\mathrm{Ca}$ show some evidence for subluminal outward motion. Although $\mathrm{C} 4$ shows apparent superluminal motion at $15 \mathrm{GHz}$, combining data at all frequencies gives an apparent speed consistent with zero. We find significant apparent superluminal outward motion of the faint component B3. C8 and C12 show some evidence of apparent inward motion. For these two components fewer epochs having well-constrained positions are available. In the following we concentrate on the inner components. 
Table 3. Linear regression fits to the motion of listed components.

\begin{tabular}{|c|c|c|c|c|c|c|}
\hline Comp. ID & $r_{\text {mean }}[\mathrm{mas}]$ & $\mu_{\mathrm{r}}^{15}\left[\mathrm{mas} \mathrm{yr}^{-1}\right]$ & $\beta_{\text {app }}^{15}[c]$ & $\mu_{\mathrm{r}}^{\mathrm{all}}\left[\mathrm{mas} \mathrm{yr}^{-1}\right]$ & $\beta_{\text {app }}^{\text {all }}[c]$ & $\mathrm{t}_{0}[\mathrm{yr}]$ \\
\hline $\mathrm{C} 0$ & $0.30 \pm 0.02$ & $0.017 \pm 0.001$ & $0.39 \pm 0.02$ & $0.006 \pm 0.001$ & $0.14 \pm 0.02$ & $T$ \\
\hline $\mathrm{C} 1$ & $0.79 \pm 0.03$ & $-0.037 \pm 0.003$ & $-0.86 \pm 0.07$ & $0.001 \pm 0.001$ & $0.02 \pm 0.02$ & / \\
\hline $\mathrm{Ca}$ & $1.27 \pm 0.03$ & $0.007 \pm 0.004$ & $0.16 \pm 0.09$ & $0.022 \pm 0.001$ & $0.51 \pm 0.02$ & / \\
\hline $\mathrm{C} 2$ & $1.93 \pm 0.03$ & $0.017 \pm 0.007$ & $0.39 \pm 0.16$ & $-0.002 \pm 0.001$ & $-0.05 \pm 0.02$ & 1 \\
\hline $\mathrm{C} 4$ & $3.70 \pm 0.08$ & $0.145 \pm 0.017$ & $3.37 \pm 0.39$ & $0.001 \pm 0.001$ & $0.02 \pm 0.02$ & 1 \\
\hline $\mathrm{C} 8$ & $6.78 \pm 0.18$ & $-0.182 \pm 0.005$ & $-4.23 \pm 0.12$ & $-0.182 \pm 0.005$ & $-4.23 \pm 0.12$ & / \\
\hline $\mathrm{C} 12$ & $10.02 \pm 0.38$ & $-0.715 \pm 0.113$ & $-16.62 \pm 2.63$ & $-0.197 \pm 0.069$ & $-4.58 \pm 1.60$ & 1 \\
\hline B3 & I & $0.807 \pm 0.151$ & $18.76 \pm 3.51$ & 1 & 1 & $1999.8 \pm 1.1$ \\
\hline
\end{tabular}

Notes. $r_{\text {mean }}$ is the mean core separation over the time-range of the regression, $\mu$ is the proper motion of the component, and $\beta_{\text {app }}$ is the apparent speed. $\mu$ and $\beta_{\text {app }}$ are computed twice: once using only $15 \mathrm{GHz}$ results, and once with the combined results from all frequencies. $t_{0}$ is the extrapolated time of component ejection from the core in the case of component B3.

\subsubsection{Comparison with geodetic VLBI data}

Britzen et al. (2005a) presented an analysis of the kinematics in the pc-scale jet of S5 $1803+784$ based on geodetic VLBI data. Such geodetic observations are performed in general more frequently than astronomical observations (please find details in Britzen et al. 2005). In order to have comparable numbers of observations per time from astronomical and geodetic observations, we smoothed the geodetic model-fit results at $8.4 \mathrm{GHz}$ in the period 1986.21-1993.95 with the following sliding mean algorithm. The new data points were produced, replacing the set of circular Gaussian parameters $m_{i}$ for each time $t_{i}$ with the weighted mean:

$m_{i}^{\prime}=\frac{1}{\Sigma p_{j}} \sum_{j=1}^{k} p_{j} m_{j}$,

where $k$ is the number of data points within interval $\left[t_{i}-\Delta, t_{i}+\Delta\right]$, and the weight of $j$ th point is determined as

$p_{j}=\exp \left[-\left(\delta t_{j} / \Delta\right)^{2}\right]$,

where $\delta t_{j}$ is the time span from the $j$ th point to the center of the window. For our analysis we selected a window value of $\Delta=$ 0.3 years, which is consistent with the temporal resolution of data points obtained at other frequencies.

\subsubsection{Flux-density evolution along the jet}

Figure 1 panel (f) shows the 15-GHz flux-density as function of distance along the jet out to $r=2$ mas, with the various epochs of observations overplotted. We find maxima in the observed distribution at $\sim 0.9$ mas and 1.4 mas. For the component at 1.4 mas we find a trend towards a sharp peak in flux-density.

\subsubsection{Displacement of $\mathrm{Ca}$}

Figure 1 panel (e) shows the position of component $\mathrm{Ca}$ in rectangular coordinates. The different symbols denote frequency $(8.4$ and $15 \mathrm{GHz})$, with the $8.4 \mathrm{GHz}$ results further divided into pre- and post- 1995.0 sets. The latter $8.4 \mathrm{GHz}$ data are obtained from the literature. These two $8.4 \mathrm{GHz}$ data-sets seem to have a shift in right ascension of $\sim 0.3$ mas. We are comparing here geodetic data with astronomical data. In the case of the geodetic data (Britzen et al. 2005a), we assumed that the brightest component is identical with component $\mathrm{Ca}$. We investigate this effect in the scenario of a Binary Black Hole in more detail (Roland et al. 2008).

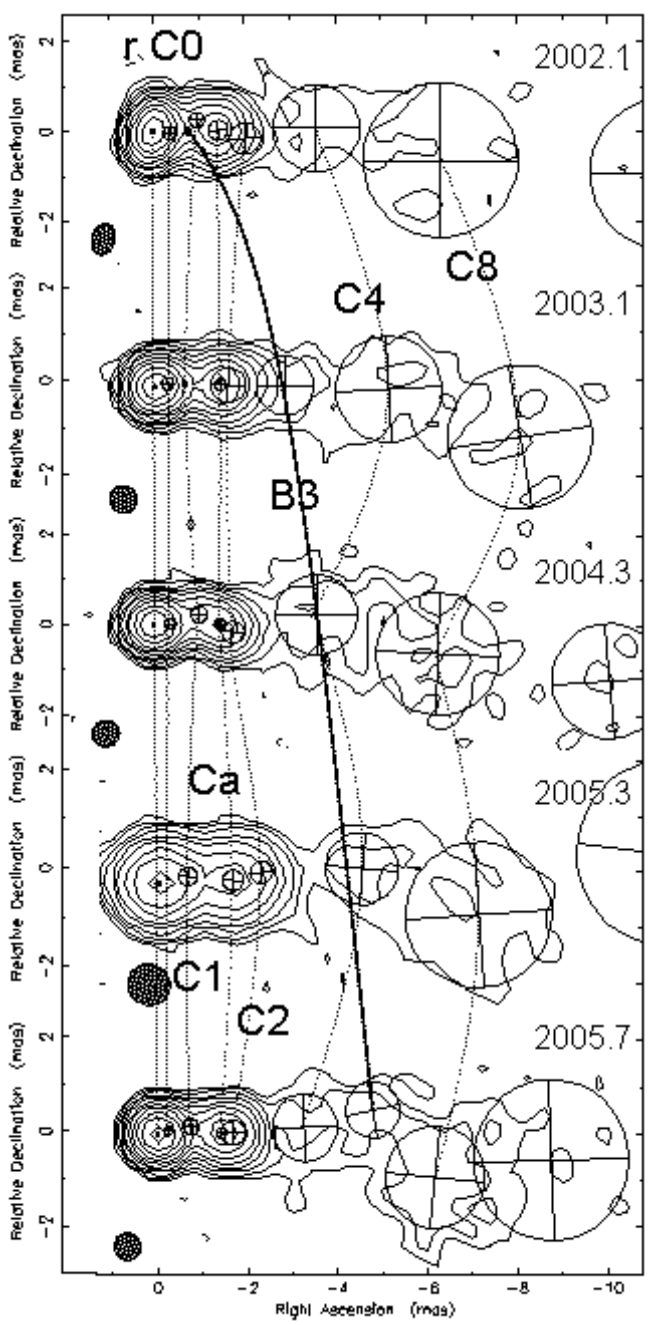

Fig. 2. Model fits superimposed on the hybrid maps obtained from Cleaning and self-calibration for the most recently observed five epochs. The component positions are connected by dotted lines. The positions of the fast component B3 are connected by a solid line. Please note that the elapsed time between adjacent epochs differs.

\subsection{The "fast" component B3}

In addition to the stationary components, one fast moving component (B3) has been observed in the time between 2002.1 and 2005.7. As shown in Fig. 1 panel (a) and Fig. 2, this component shows outward motion with an apparent velocity of $\sim 19 c$ 
Table 4. Gaussian parameters of outbursts in the UMRAO light-curves.

\begin{tabular}{lrlllr}
\hline \hline Comp. & $\begin{array}{r}\text { Freq. } \\
\text { GHz }\end{array}$ & Amplitude & $\begin{array}{l}T_{\max } \\
\mathrm{yr}\end{array}$ & $\begin{array}{l}\Theta \\
\mathrm{yr}\end{array}$ & $\begin{array}{r}\text { Time delay } \\
\mathrm{yr}\end{array}$ \\
\hline $\mathrm{A}$ & 8.0 & $1.94 \pm 0.02$ & $1985.19 \pm 0.02$ & $1.41 \pm 0.02$ & $0.00 \pm 0.02$ \\
$\mathrm{~A}$ & 4.8 & $0.77 \pm 0.04$ & $1985.39 \pm 0.04$ & $1.41 \pm 0.04$ & $0.20 \pm 0.06$ \\
$\mathrm{~B}$ & 14.5 & $2.01 \pm 0.01$ & $1988.93 \pm 0.01$ & $2.63 \pm 0.01$ & $0.00 \pm 0.01$ \\
$\mathrm{~B}$ & 8.0 & $2.15 \pm 0.02$ & $1988.86 \pm 0.01$ & $4.07 \pm 0.02$ & $-0.07 \pm 0.02$ \\
$\mathrm{~B}$ & 4.8 & $1.25 \pm 0.01$ & $1989.19 \pm 0.01$ & $2.54 \pm 0.01$ & $0.26 \pm 0.02$ \\
$\mathrm{C}$ & 14.5 & $1.46 \pm 0.01$ & $1996.94 \pm 0.02$ & $9.83 \pm 0.03$ & $0.00 \pm 0.02$ \\
$\mathrm{C}$ & 8.0 & $1.57 \pm 0.02$ & $1998.34 \pm 0.04$ & $7.89 \pm 0.04$ & $1.40 \pm 0.06$ \\
$\mathrm{C}$ & 4.8 & $0.93 \pm 0.01$ & $2000.13 \pm 0.04$ & $6.28 \pm 0.05$ & $3.20 \pm 0.06$ \\
\hline
\end{tabular}

based on 3 epochs of observations. B3 is much fainter (between 0.01 and $0.04 \mathrm{Jy}$ ) and shows a kinematical behavior that differs significantly from the other components, which don't show long-term secular outward motion. The ejection time of this component computed by extrapolating it's proper motion back to $r=0$ is $1999.8 \pm 1.1$. Unfortunately, this epoch is in the middle of the two-year gap in the observations.

\subsection{Further evidence for the coexistence of one "fast" component among "stationary" components}

In order to check our model of the S5 $1803+784$ jet, in which only one component moves with apparent superluminal speed, we investigated in detail the opacity and spectral evolution of the total flux-density light-curves. The emergence of a new jet component, according to the shock-in-jet model (e.g., Marscher 1996; Gomez et al. 1997), is caused by a shock induced by a primary excitation at the base of the jet. This manifests itself in radio light-curves as an outburst that is delayed at lower frequencies due to the combined effects of the frequency stratification of the emitting electrons, non-zero opacity, and light-travel delays. Such time-delayed outbursts are associated observationally on mas-scales with the brightening of the VLBI core and are accompanied by the ejection of a new optically thin jet component.

We decomposed the total flux-density light-curves at 4.8 GHz, $8 \mathrm{GHz}$, and $14.5 \mathrm{GHz}$ from the University of Michigan Radio Observatory (UMRAO) monitoring campaign (Aller et al. 1999, 2003) into Gaussian components, as was described in Pyatunina et al. (2006, 2007). Figure 3 shows the light-curve of S5 $1803+784$ at $14.5 \mathrm{GHz}$ and the resulting Gaussian decomposition. Three prominent outbursts are visible: (A) in 1985; (B) in 1988, and (C), a very prolonged outburst starts in $\sim 1992$ and ends in $\sim 2005$ with a peak in 1997. The Gaussian parameters, fitted to the light-curve are listed in Table 4. Frequencydependent time delays for each flare are the time difference between the Gaussian peak at each frequency with respect to the position of the peak at $14.5 \mathrm{GHz}$. These are plotted in Fig. 4. Flares A and B show moderate time delays of 0.3 years, whereas the prolonged flare $\mathrm{C}$ shows an enormous time delay of 3.2 years between $4.8 \mathrm{GHz}$ and $14.5 \mathrm{GHz}$, which is an indication of high opacity in the source during the $\mathrm{C}$ outburst and a flat spectrum that becomes gradually steep. Outburst $\mathrm{C}$ has a very broad profile at all frequencies $(\Theta \sim 8$ yr). Similarly broad outbursts were observed for the source 1308+326 in Pyatunina et al. (2007), where it was mentioned that such a broad outburst can be associated with the development of a dense region, some kind of "cocoon" that confines the innermost part of the jet that is excited by the primary perturbation. We calculated quasi-simultaneous spectra using the measurements at all three frequencies for which time separation is less than two weeks; Fig. 5 shows the

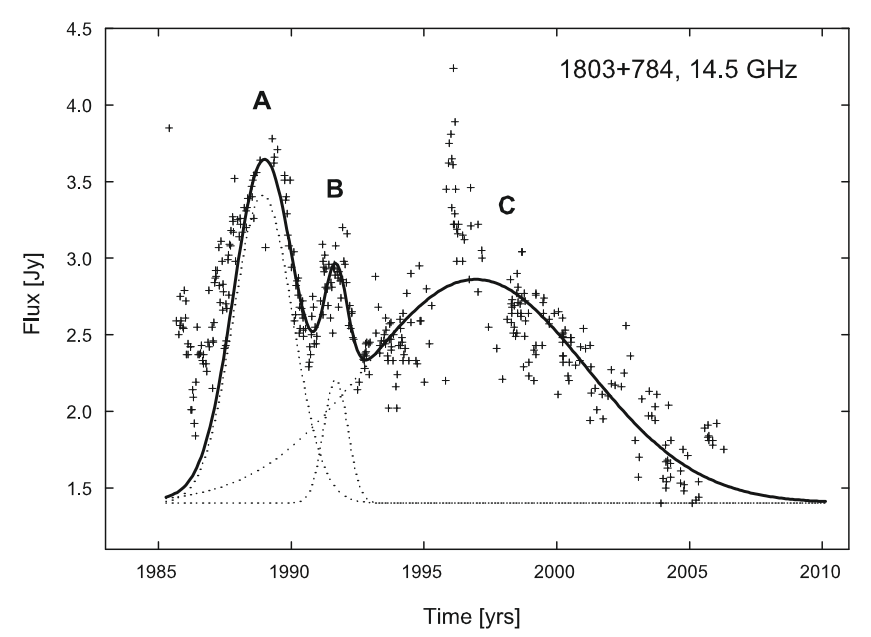

Fig. 3. Gaussian decomposition of the UMRAO $14.5 \mathrm{GHz}$ light-curve. The solid line shows the sum of the Gaussian functions and dotted lines are individual Gaussian functions. Letters indicate the names of the flares.

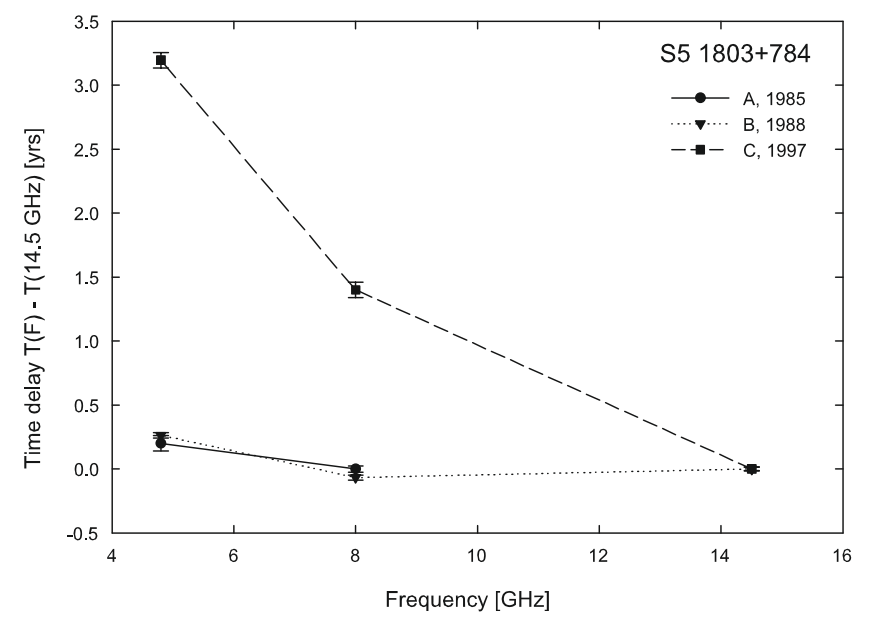

Fig. 4. Time lags of individual outbursts as functions of frequency.

spectral evolution fit as a power law to each spectrum. The spectral behavior changes significantly after $\sim 1996$, where the spectra change from being flat, and gradually become steeper. The changes of the spectral evolution occur in the beginning of a bright, prolonged flare $\mathrm{C}$ and can be associated with it. We suggest that this can be explained by a jet component ejection. Small frequency-dependent time delays and steep spectra of other observed flares of the source suggest that there probably were no jet component ejections before 1995 which is in good agreement with our model for the S5 1803+784 jet. 


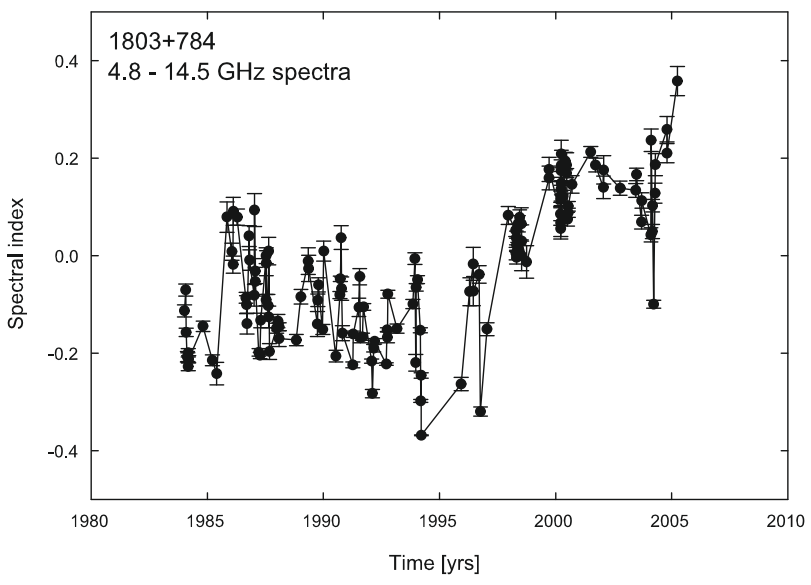

Fig. 5. Time evolution of the quasi-simultaneous spectral index, fit to the $4.8 \mathrm{GHz}, 8 \mathrm{GHz}$, and $14.5 \mathrm{GHz}$ flux densities.

\subsection{Significant position angle changes}

As shown before, most of the components remain at similar core separations for the time covered by the observations. Although it is obvious from the panels in Figs. $1 \mathrm{a}$ and $\mathrm{b}$ that most jet components show an additional motion with regard to the core separation superimposed on their rather stationary behavior, we concentrate in this paper on the most important aspects only. A detailed analysis of the second order motion is complicated by the uneven sampling. This effect of "oscillating" motion has been analyzed in detail for $\mathrm{Ca}$ in $1803+784$ by Britzen et al. (2005a). The phenomenon of "oscillations" is clearly seen in other components of $1803+784$ and in other BL Lac objects as well and will be analyzed in detail in Glueck et al. (in prep).

The components show significant position angle changes. Figure 6 panels (a)-(d) show the evolution of the position angle for four jet components $(\mathrm{C} 0, \mathrm{C} 1, \mathrm{C} 2$, and $\mathrm{Ca}$, respectively) at different frequencies. The position angles vary over $35^{\circ}$ in the case of $\mathrm{C} 0$ and $15^{\circ}$ in the case of $\mathrm{C} 2$. We find very similar relations at 5 and $8 \mathrm{GHz}$ for $\mathrm{C} 0$, at 5,8 , and $15 \mathrm{GHz}$ for $\mathrm{C} 1$, at 8 and $15 \mathrm{GHz}$ for $\mathrm{C} 2$, and at 5,8 , and $15 \mathrm{GHz}$ for $\mathrm{Ca}$. One outlier for C0 in Fig. 6 panel (a) in 1994 can probably be explained by a blending effect with the core or a new component due to the closeness of the core. In the case of the core separation for $\mathrm{C} 0$ and $\mathrm{C} 2$, the evolution is similar at the two frequencies. For C2 we find some indication that the lower frequency-data can be found at larger core separations.

\subsection{Evolution of the mean jet ridge line}

In Fig. 8 we show the jet ridge line for all epochs obtained at $15 \mathrm{GHz}$ in Cartesian coordinates. The jet ridge line is defined by a line connecting all component positions at a given epoch and is independent of any component identification. We find that the jet ridge line changes its shape between epochs in a periodic way. Figure 8 panel (a) shows the temporal evolution of jet component positions at $15 \mathrm{GHz}$ for the time period 1994-2005. It overplots the jet ridge lines at each epoch, with each dot representing the position of one jet component and each line connecting the jet components for one particular epoch. Panels (b) and (c) show this evolution with time in more detail; the lefthand columns showing the trace of the ridge line at each epoch, and the right-hand columns the flux density of the components along the jet as a function of $x$. From an almost straight line in 1994.67, the shape of the jet evolves into a sinusoid with the

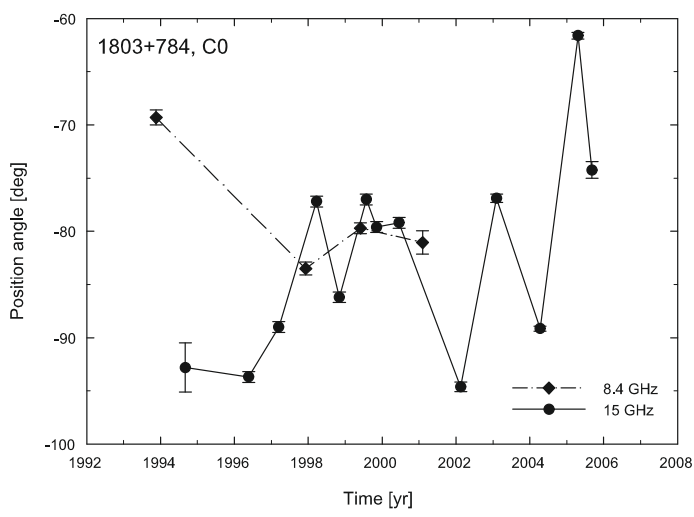

(a)

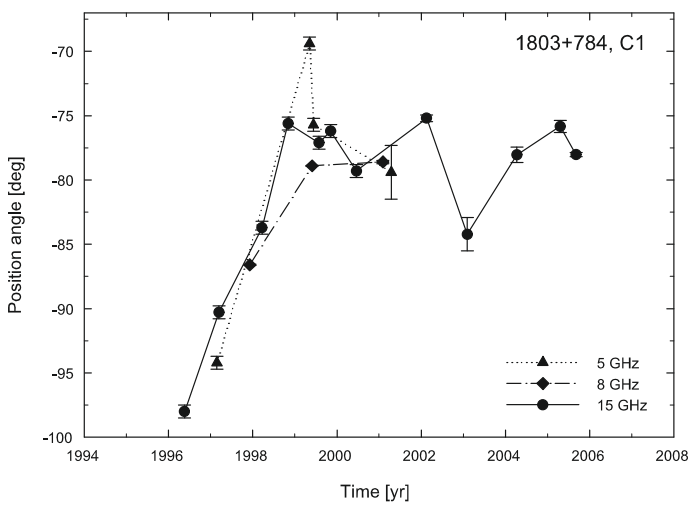

(b)

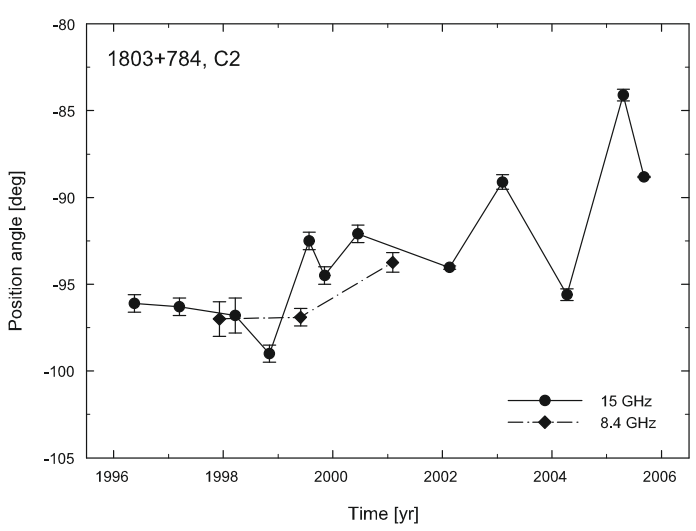

(c)

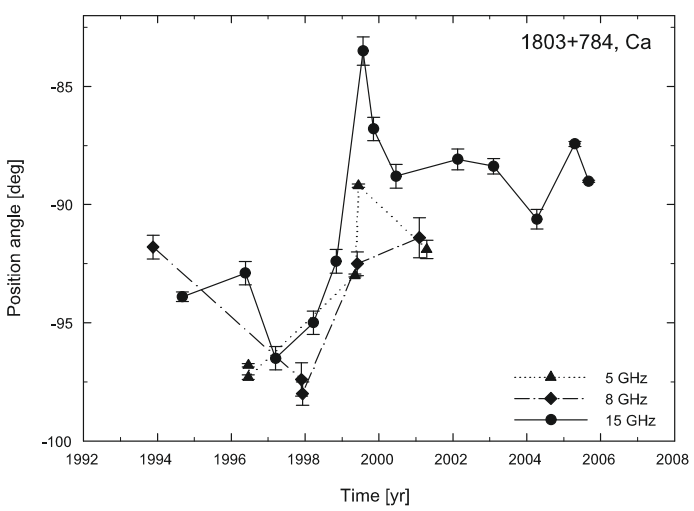

(d)

Fig. 6. Position angle as function of time at different frequencies for four individual components. 
northernmost value at $x \sim 1$ mas and the southernmost value at $x \sim 2$ mas. The amplitude of the sinusoid reaches its maximal values at epoch 1998.84 and decreases again, forming an almost straight line again in 2003.10. One period of this excursion is completed after $\sim 8.5$ years and the jet shape starts to evolve into a sinusoid again. However, the position of the straight lines in 1994.67 and 2003.10 are different, with an offset in $y$ of 0.1 mas. We thus see an evolution of the jet ridge line with time as defined by the positions of the individual components within the jet.

\subsection{Evolution of the jet width}

In this section we investigate the width of the jet as defined by the position angle distribution that the jets components span. Figure 9 shows this distribution as function of time, including all jet components seen at all observed frequencies. This plot shows that the jet changes its width with time: around 1995 the jet width appears to be quite small $\left(\sim 5^{\circ}\right)$, around 1999.5 the jet width appears to be much broader $\left(\sim 40^{\circ}\right)$ and is narrower again in 2004 . To investigate the longterm evolution of this effect, we include data taken from the literature in Fig. 9 panel (b) and find a similar evolution of the width. The complete dataset (our data + literature data) seems to cover two cycles of jet-width broadening with a period of about $8-9$ years. To investigate whether this broadening is mainly a frequency dependent effect, we show the distribution at $15 \mathrm{GHz}$ in panel (c) and at $8.4 \mathrm{GHz}$ in panel (d); both panels confirming the trend of changing jet width. To investigate whether less well-constrained model components at large core separations with large position angles produce this effect, we checked whether the shape of the plot changes when we include only jet components within a certain range of core separations. We checked the data for the jet components within the inner 1 mas, 2 mas, etc. up to 7 mas. However, the shape of the position angle distribution is stable and does not change significantly. Panels (c) and (d) discussed above show the distribution for components within 5 mas of the core.

To check whether periods of more frequent observations produce more outliers in position angle and thus affect the position angle distribution, we binned the data in 0.3 year bins and calculated the standard deviation in position angle within each bin, both for $5 \mathrm{GHz}$ and $8.4 \mathrm{GHz}$. This procedure used the components within 5 mas of the core. Figure 7 shows the evolution of the standard deviation per bin (the outliers were removed from the plot), using the center of each bin as its epoch for plotting. The position angle spread with time shows a smooth increase starting in 1985, and reaches its maximum in 1988-1992. It then decreases again and has the second maximum in about 1998. It is clearly seen that the spread of the position angle evolution has two cycles with a characteristic timescale of about 8-9 years, similar to the period suggested by the evolution of the jet ridge line. The autocorrelation function gives the value of the timescale: $9.9 \pm 0.2$ with a correlation coefficient of 0.79 . Binning the data with different bin length, such as 0.5 or 1.0 years gives the same results. In order to check whether the different number of jet components for different epochs of observations can affect these results, we selected only data points within 0.5 mas core separation. We only took the first three jet components for each particular epoch into account and binned the position angles into 0.3 year bins. The evolution of the standard deviation of the position angles in these bins looks similar. We thus conclude that the shape of the plot and characteristic time scale of the position angle changes do not depend on the number of the jet components for a particular epoch of observation.

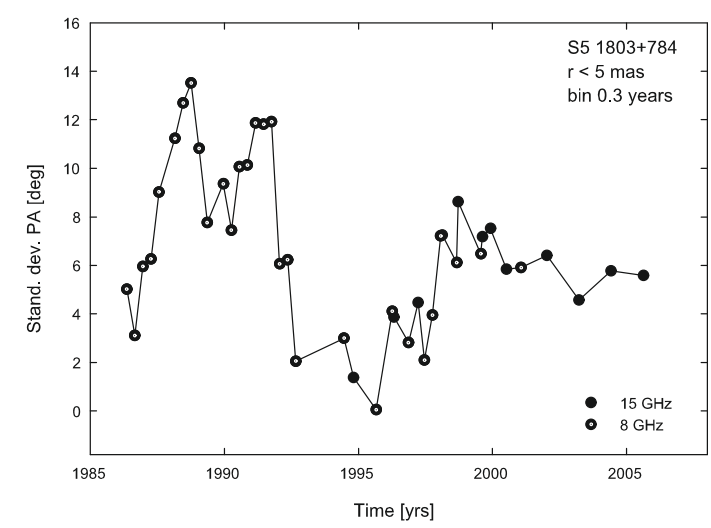

Fig. 7. Time evolution of standard deviation of position angles, calculated for components within 5 mas of the core in 0.3 year bins. Dotted circles show $8.4 \mathrm{GHz}$ data; circles $15 \mathrm{GHz}$ data.

\section{Summary: kinematic results}

Before we describe the periodicities and correlations found in S5 1803+784, we give a brief summary of the results of the kinematic analysis. In Britzen et al. (2005a) we presented a component identification scenario for the pc-scale jet in S5 1803+784 based on the assumption that the brightest component seen in each observing epoch can be identified with the same component $(\mathrm{Ca})$. The most plausible identification scenario resulting from this assumption was the one presented in that paper: the coexistence of several superluminally moving and one oscillating component.

The data presented in this paper cover a broader range in frequency (1.6-15 GHz and time (1993.88-2005.68). Thus, a more detailed investigation of the jet component motion is possible. In addition, we collected kinematic information from the literature and tested our hypothesis against these data. In total, 94 data sets have been investigated. Conclusions derived from the present analysis include:

- most jet components within the inner $\sim 10$ mas of the core remain at roughly constant core separation over the long term (see Fig. 1);

- the position angles of these components change significantly over time (see Fig. 6);

- component (B3) moves with a superluminal apparent speed of $\sim 19 c$ based on 3 epochs (see Figs. 1 panel (a) and 2);

- the jet ridge line evolves with time, with a likely period of $\sim 8.5$ years (see Fig. 8), independent of any component identification;

- the width of the jet changes periodically with a similar period of 8-9 years (see Fig. 9).

In the following section we show that the epochs of the maximum widths of the position-angle distribution correlate well with maxima in the total flux density observed within the UMRAO monitoring programme (see Figs. 10 and 11). We find convincing correlations between parameters such as the flux-density, core separation, position angle, and describe them below.

\section{Correlations and periodicities}

\subsection{Correlations and anti correlations}

As shown in previous sections, the jet width changes with time and the jet ridge line itself shows a quasi-periodic variation 
S. Britzen et al.: The kinematics in the pc-scale jets of AGN

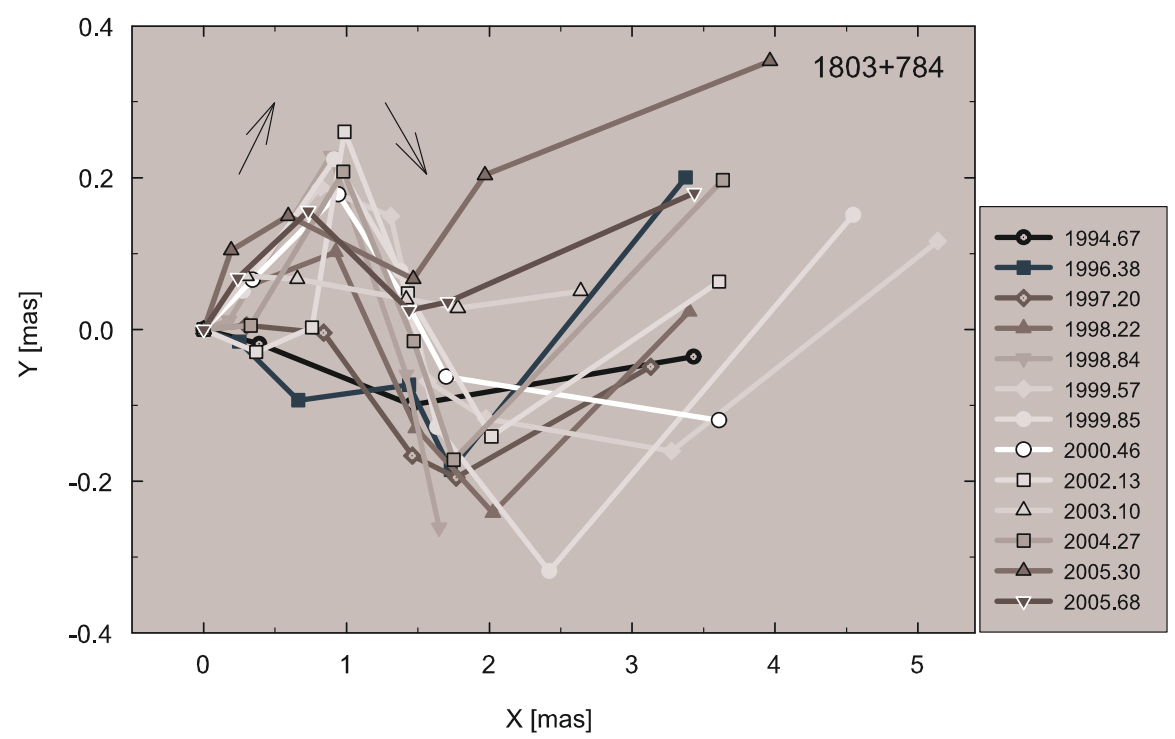

(a)

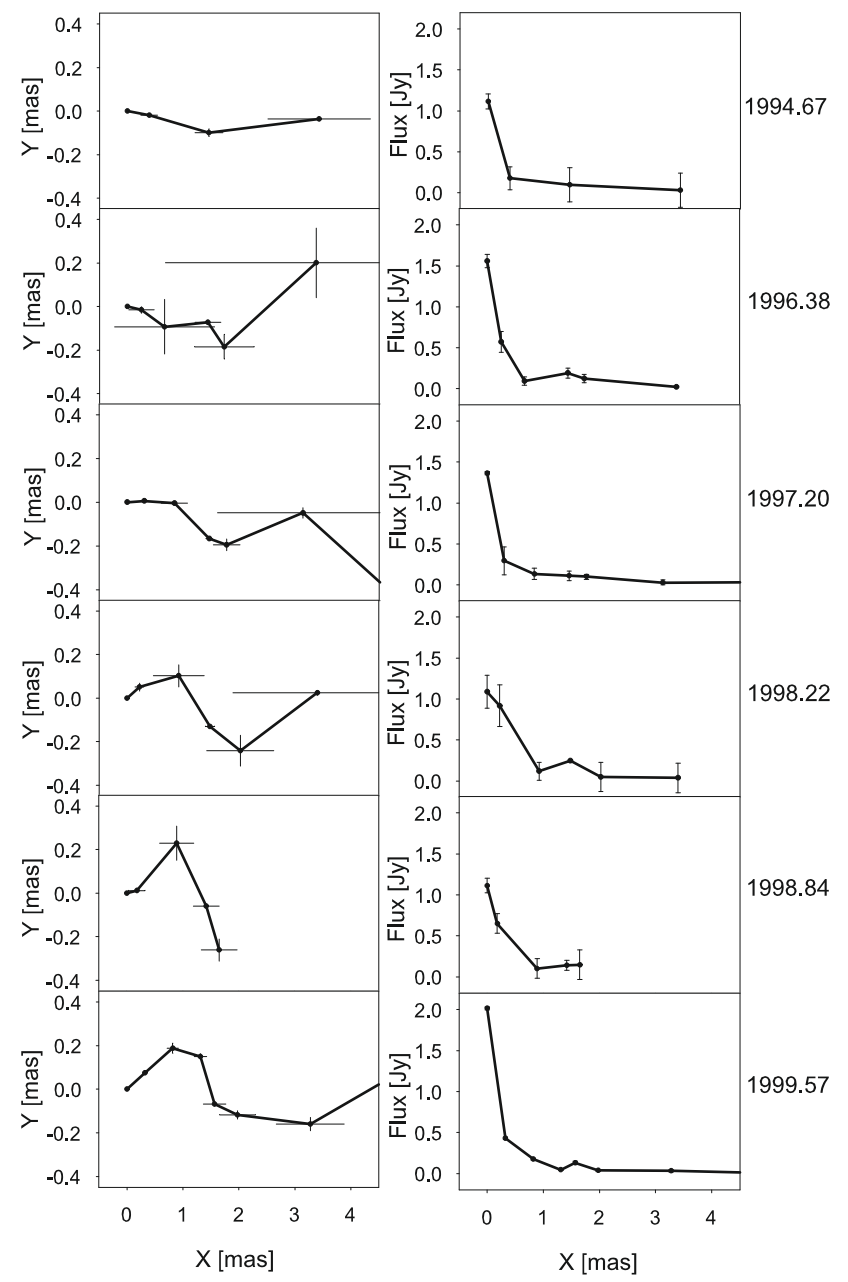

(b)

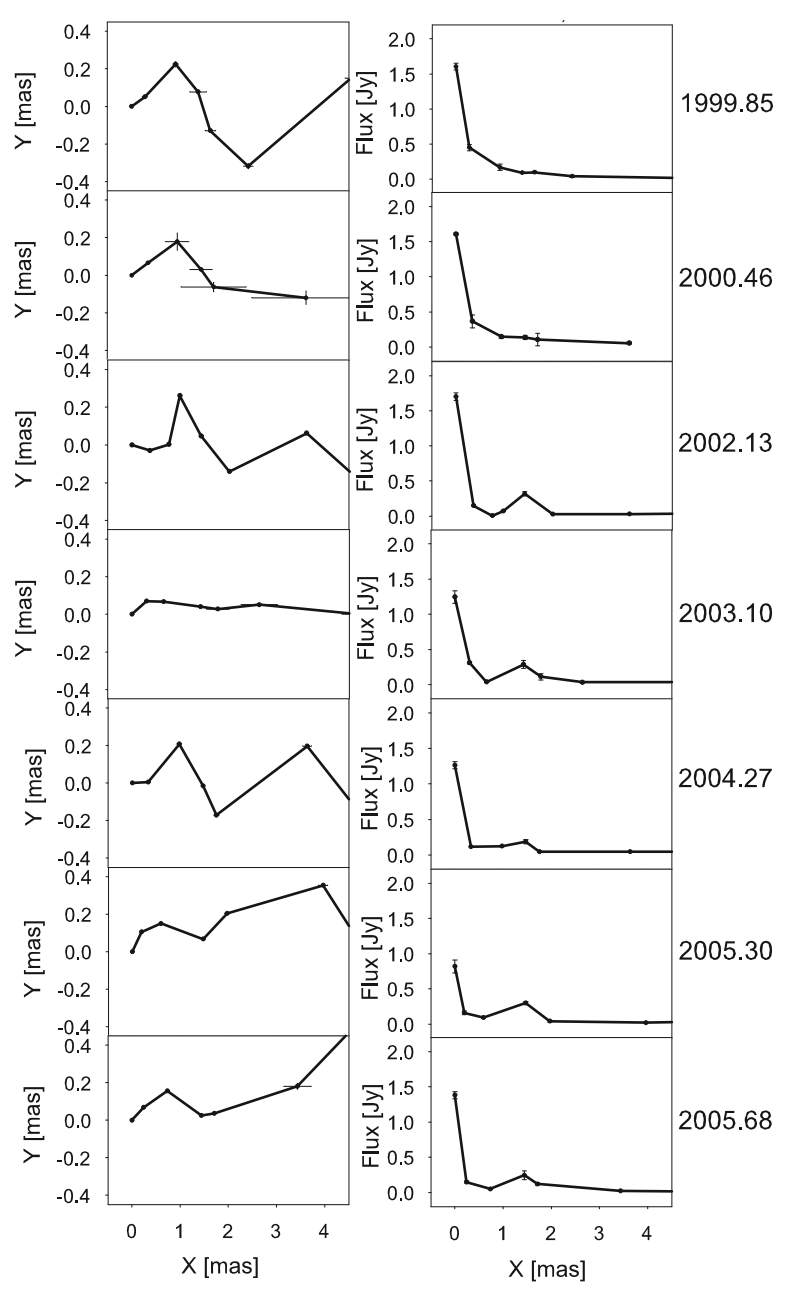

(c)

Fig. 8. The jet ridge line for all components detected in $15 \mathrm{GHz}$ observations within 6 mas of the core. Panel a) overplots all epochs, as denoted by the different lines/points. Panels b) and c) show the trace of the jet in Cartesian coordinates (left-hand columns) and the flux density along the jet as a function of $x$ (right-hand columns).

with a characteristic time scale of $\sim 8.5$ yrs. Both facts could result from a geometric origin. This would also produce correlation among the core separation, the position angle, and the flux density changes for each jet component and across the different components. To investigate these correlations, we first performed a visual analysis of the time series of the position 


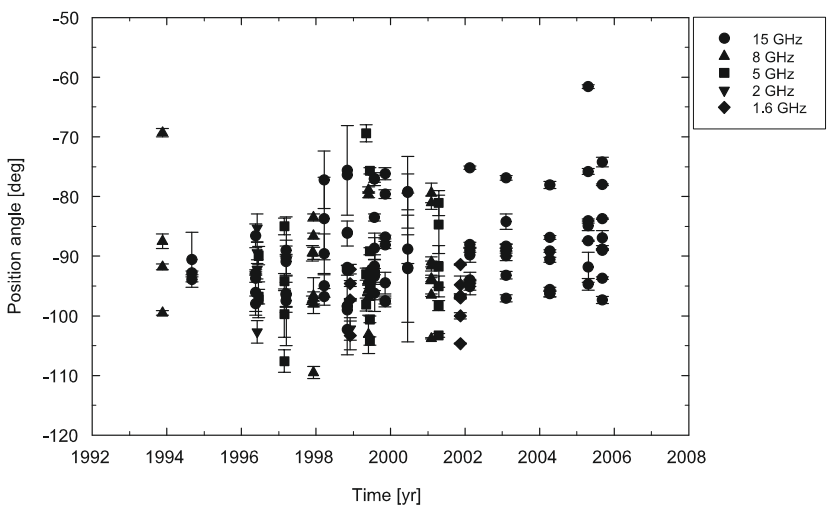

(a)

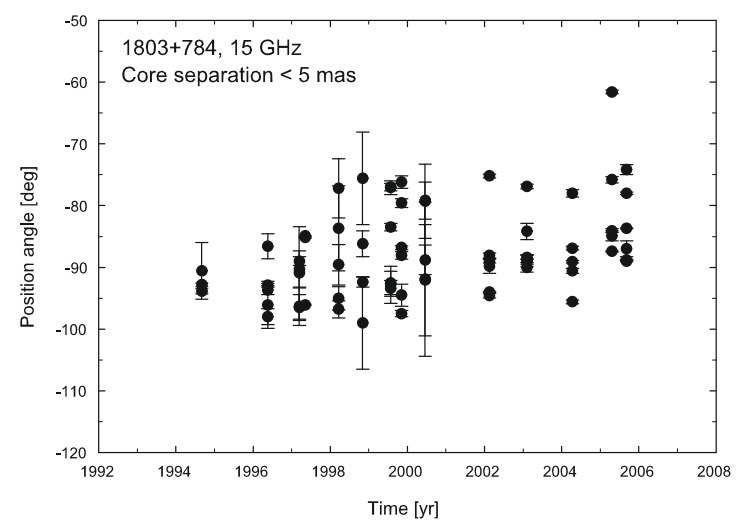

(c)

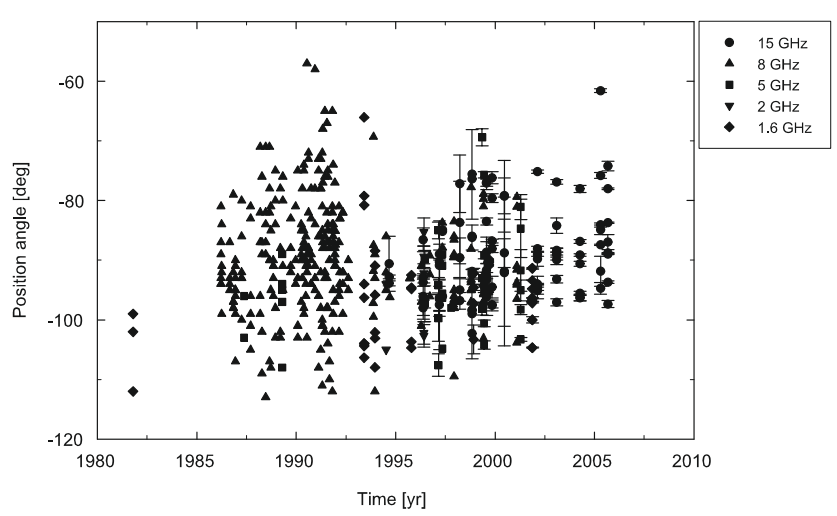

(b)

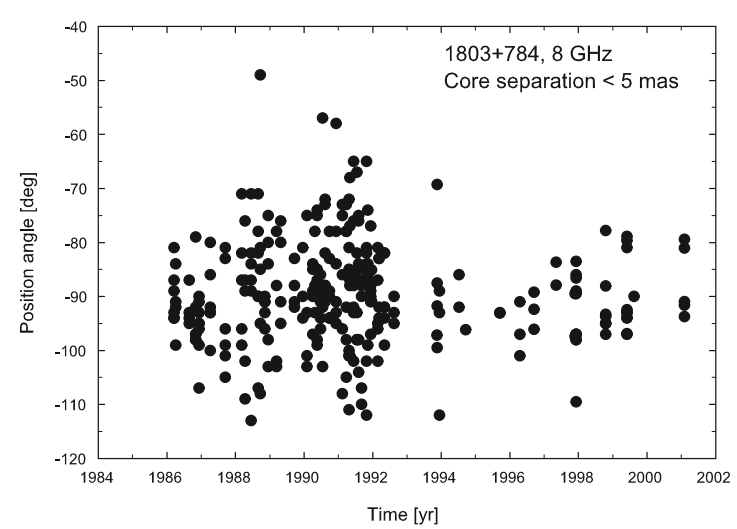

(d)

Fig. 9. Position angle distribution of individual jet components as a function of time. Panels a) and b) show the new observations from this paper and those combined with the observations from the literature, respectively, for all frequencies. Panels c) and d) show components within 5 mas of the core $15 \mathrm{GHz}$ and $8.4 \mathrm{GHz}$, respectively.

angle, core separation, and the flux of each component at 8.4 and $15 \mathrm{GHz}$. Maxima and minima at $8.4 \mathrm{GHz}$ are listed in Table 5. Major changes in the variations of the jet parameters at $8 \mathrm{GHz}$ occurred in 1987, 1988.5, 1990 and 1991 for C0, C1, Ca and the core. The core flux reached a maximum in 1987, accompanied by minima in flux, position angle, and core separation of $\mathrm{C} 1$, maxima of the core separation of $\mathrm{C} 0$ and maxima for flux and position angle of Ca. In 1988.5, the values that were maximal became minimal and vice versa, and switched again in 1990 and 1991. This indicates that changes in flux, position angle and core separation of the components and the core-flux are correlated and change with characteristic time scales of a few years. Similar behavior was found as well at $15 \mathrm{GHz}$ at two other epochs (Table 6). The major changes in core separation, position angle, and flux variations of the inner components occurred in 1998 and 2000.

To investigate the correlations among these circular Gaussian parameters of a component and across multiple components more carefully, we calculated the discrete cross-correlation functions (Edelson \& Krolik 1988) for the pairs of parameters (core separation, flux density, position angle) for each of the components $(\mathrm{C} 0, \mathrm{C} 1, \mathrm{Ca}, \mathrm{C} 2, \mathrm{C} 4)$ at $8.4 \mathrm{GHz}$. Component $\mathrm{C} 2$ at $15 \mathrm{GHz}$ possibly comprises 3 components at $8.4 \mathrm{GHz}$. We call these three $\mathrm{C} 2$ components at $8.4 \mathrm{GHz} \mathrm{C} 2_{1}, \mathrm{C}_{2}$, and $\mathrm{C} 2_{3}$. We find the following results:

- For the brightest jet components $\mathrm{Ca}$ and $\mathrm{C} 1$, all the parameters are correlated. As an example, Fig. 12 shows the time series of the core separation, the position angle, and the flux density of the component C1 from 1984 to 1996.

- For the jet components $\mathrm{C} 2_{1}, \mathrm{C} 2_{2}$ and $\mathrm{C} 4$, we find a strong correlation or anti-correlation among the variations in flux density, position angle, and core separation within each component. Table 7 shows the discrete correlation function peaks for these parameters in jet components.

- The core flux is correlated with the core separation, the flux density, and the position angle changes of all inner jet components $\mathrm{C} 0, \mathrm{C} 1, \mathrm{Ca}, \mathrm{C} 2{ }_{1}, \mathrm{C} 2_{2}$ and $\mathrm{C} 2{ }_{3}$ (see Table 8).

- Variations of individual circular Gaussian parameters also show correlations across different jet components. Table 8 also shows the results of the discrete correlation function calculation.

When cross-correlating core separation changes of $\mathrm{Ca}$ and those of other components, significant peaks in the discrete correlation function (e.g., $\mathrm{C} 0, \mathrm{C} 1, \mathrm{C} 2_{1}$ and $\mathrm{C} 2{ }_{3}$ ) are associated with time lags that change gradually with distance along the jet. The time lags appear to increase roughly linearly with core separation; Fig. 14 shows the time lags for these component pairs as a function of the components' mean core separation (time lags for components interior to $\mathrm{Ca}$ have had their sign inverted from Table 8), and fit with a linear regression. Similar behavior is found for the cross-correlation between the core separation changes of $\mathrm{C} 1$ and $\mathrm{Ca}, \mathrm{C} 2_{1}, \mathrm{C} 2_{2}$, and $\mathrm{C} 2_{3}$. This can be explained if we assume that the motion of the components is mostly due to a geometrical origin. The components follow 
Table 5. Maxima and minima in flux density, position angle and core separation at epochs 1987, 1988.5, 1990, and 1991 at 8.4 GHz.

\begin{tabular}{|c|c|c|c|c|c|c|c|c|c|c|c|c|}
\hline \multirow{2}{*}{$\begin{array}{c}\text { Time } \\
\text { Comp. ID }\end{array}$} & \multicolumn{3}{|c|}{1987} & \multicolumn{3}{|c|}{1988.5} & \multicolumn{3}{|c|}{1990} & \multicolumn{3}{|c|}{1991} \\
\hline & Flux & $\mathrm{PA}$ & Core sep. & Flux & PA & Core sep. & Flux & PA & Core sep. & Flux & $\mathrm{PA}$ & Core sep. \\
\hline Core & $\max$ & & & $\min$ & & & $\max$ & & & $\min$ & & \\
\hline $\mathrm{C} 0$ & & & $\max$ & & & $\min$ & & & $\max$ & $\min$ & & $\min$ \\
\hline $\mathrm{C} 1$ & $\min$ & $\min$ & $\min$ & $\max$ & $\max$ & $\max$ & $\min$ & $\min$ & $\min$ & $\max$ & $\max$ & $\max$ \\
\hline $\mathrm{Ca}$ & $\max$ & $\max$ & $\min$ & $\min$ & $\min$ & $\max$ & $\max$ & $\max$ & $\min$ & & & \\
\hline
\end{tabular}

Table 6. Maxima and minima in flux density, position angle and core separation at epochs 1998 and 2000 at $15 \mathrm{GHz}$.

\begin{tabular}{lcccccr}
\hline \hline \multicolumn{1}{c}{ Time } & \multicolumn{2}{c}{1998} & & \multicolumn{2}{c}{2000} \\
Comp. ID & Flux & PA & Core sep. & Flux & PA & Core sep. \\
\hline Core & min & & \multirow{2}{*}{$\max$} & & \\
C0 & max & max & min & & & \\
C1 & & & & $\max$ & $\max$ & $\max$ \\
$\mathrm{Ca}$ & $\max$ & $\min$ & $\max$ & $\min$ & $\max$ & $\min$ \\
\hline
\end{tabular}

almost the same trajectories, but with a certain time delay. The components are connected with each other and the information is spreading with a proper motion of about $2.3 \mathrm{mas} / \mathrm{yr}$ if the reference point is component $\mathrm{Ca}$ and $0.79 \mathrm{mas} / \mathrm{yr}$ if the reference point is component $\mathrm{C} 1$, corresponding to extremely fast speeds of $53 c$ and $18.2 c$, respectively.

We investigated possible correlations among the jetcomponent parameters and the total flux-density variability. For this purpose we used the UMRAO data of multi-frequency AGN monitoring (Aller et al. 1999, 2003), spanning almost 30 years (see Fig. 11). Using the $8 \mathrm{GHz}$ UMRAO light-curve over the time-range spanned by the VLBI data, we find:

- variations in the core separation and position angle of $\mathrm{Ca}$ and $\mathrm{C} 2{ }_{1}$ are correlated with the total flux-density variability (see Table 7);

- variation in the core separation and the position angle changes of the jet component $\mathrm{C} 1$ are correlated with the total flux density at $14.5 \mathrm{GHz}$ (see Fig. 12), but do not show any correlation at $8 \mathrm{GHz}$.

In order to investigate the same correlations at $15 \mathrm{GHz}$ where the number of data points was not sufficient for the discrete correlation function analysis, we calculated the Pearson's correlation coefficient and inspected them visually. Tables 7 and 8 show the Pearson correlation coefficients and the probabilities for finding such a value by chance for various pairings of different parameters for a single component and the same parameter across two components, respectively. The flux-density variability is anti-correlated for the pairs of $\mathrm{C} 1 / \mathrm{Ca}$, and $\mathrm{Ca} / \mathrm{C} 2$. We only find correlated core separation changes for the pair $\mathrm{Ca} / \mathrm{C} 2$. Figure 13 shows notable examples for a correlation and anticorrelation: the position angle vs. core separation in component $\mathrm{Ca}$ (panel a) and the flux densities in components $\mathrm{Ca}$ vs. C1 (panel b), respectively.

\subsection{Periodicities}

To test for possible periodic changes in the component parameter variations, we applied a discrete autocorrelation function (Edelson \& Krolik 1988), a data-compensated discrete Fourier transform (Ferraz-Mello 1981), and the Jurkevich method (Jurkevich 1971). All three methods were applied over the timerange 1981-2005 for all components out to C12. We find a

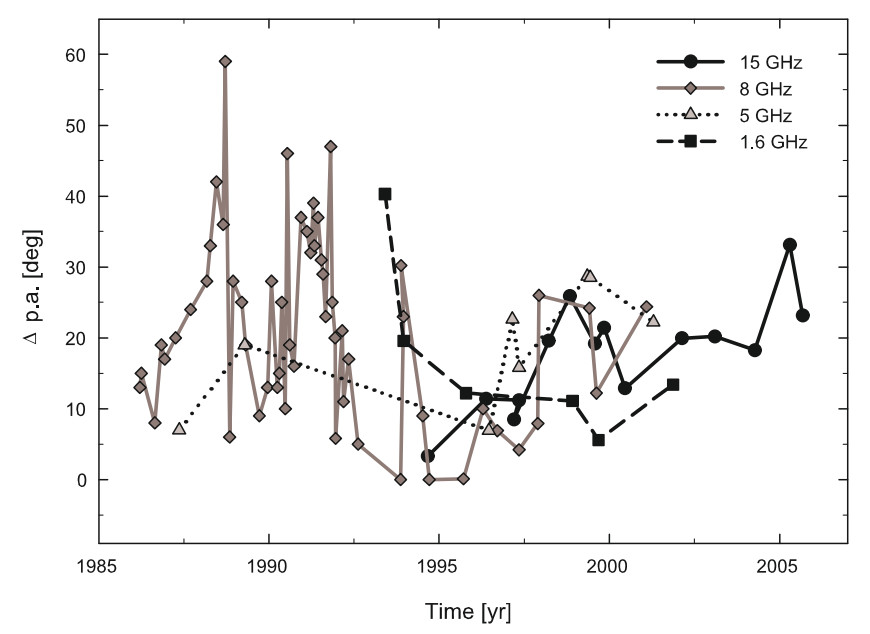

Fig. 10. The spread in the position angle for four different frequencies as a function of time, based on all the data (this paper + literature). We clearly find several maxima that are correlated with the maxima found in the total flux-density data.

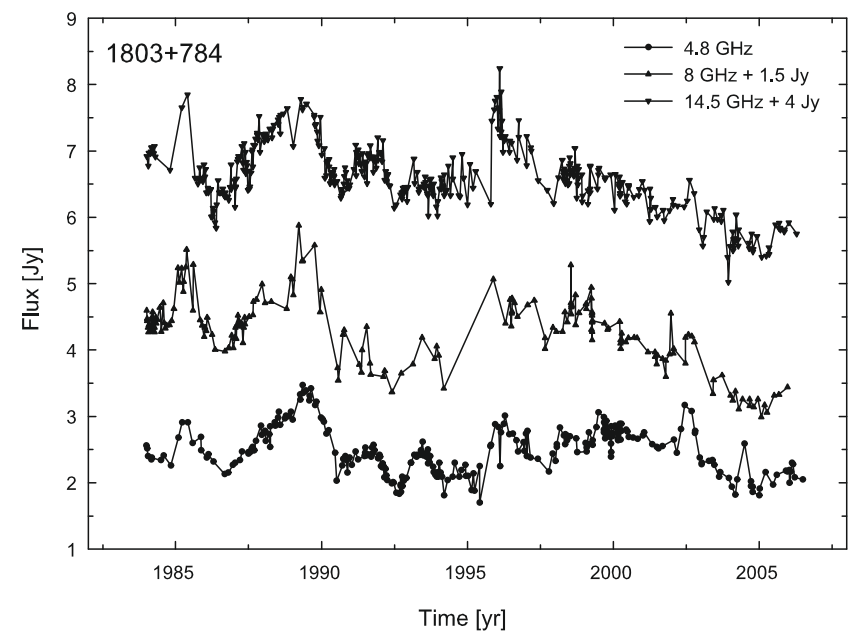

Fig. 11. Total single-dish flux-density light-curve for S5 $1803+784$ at $4.8,8$ and $14.5 \mathrm{GHz}$. The 8 and $14.5 \mathrm{GHz}$ light-curves are shifted by 1.5 and $4 \mathrm{Jy}$, respectively. All the data are from the UMRAO monitoring campaign.

quasi-periodicity of about 2 and 4 years in the variation of the core separation, the position angle, and the flux with time for the inner jet components $\mathrm{C} 0, \mathrm{C} 1, \mathrm{Ca}$, and $\mathrm{C} 2$ at 8 and $15 \mathrm{GHz}$. This agrees well with the periods of 2 and 3.9 years found by Kelly et al. (2003) in the total flux density variability. A more detailed description of this analysis will be presented by Kudryavtseva et al. (in prep.). 


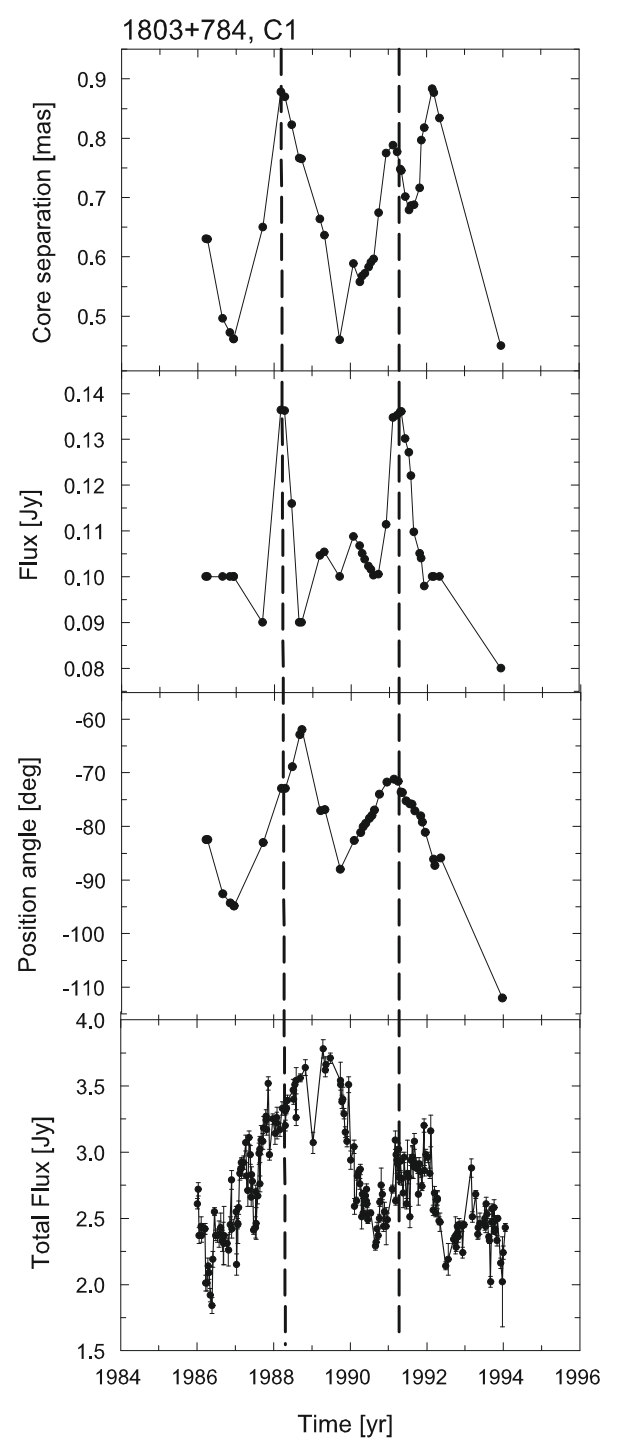

Fig. 12. Core separation, flux density, position angle of component $\mathrm{C} 1$, and total flux-density light-curve at $8 \mathrm{GHz}$ in the period 1984-1996. The two dashed lines indicate the nearly simultaneous peaks in all four plots.

\section{Discussion}

In this paper we have presented multi-frequency kinematic analysis for S5 1803+784 based on observations covering roughly twenty years in time. The most important result is that most of the features in the pc-scale jet up to a core distance of $\sim 12$ mas remain at roughly constant core separations over long time scales. This is in contrast to most previously presented kinematic scenarios for this source or flat-spectrum AGN in general. While many authors find that component $\mathrm{Ca}$ is stationary, most authors claim outward motion for the remaining components. In contrast, the $2 \mathrm{~cm} / \mathrm{MOJAVE}$ webpage and Lister et al. (2009) show a core separation/time plot in which several components within $\sim 8$ mas of the core also maintain roughly constant core separations. This is consistent with our results presented in this paper. The $2 \mathrm{~cm}$ data from the $2 \mathrm{~cm} / \mathrm{MOJAVE}$ survey form part of the literature data sets included in the present analysis. The fast-moving component B3 is not seen by the $2 \mathrm{~cm} /$ MOJAVE.

The combination of new and re-analyzed data spanning 12-20 years shows that all bright components of the inner jet remain at roughly constant core separations, with no significant

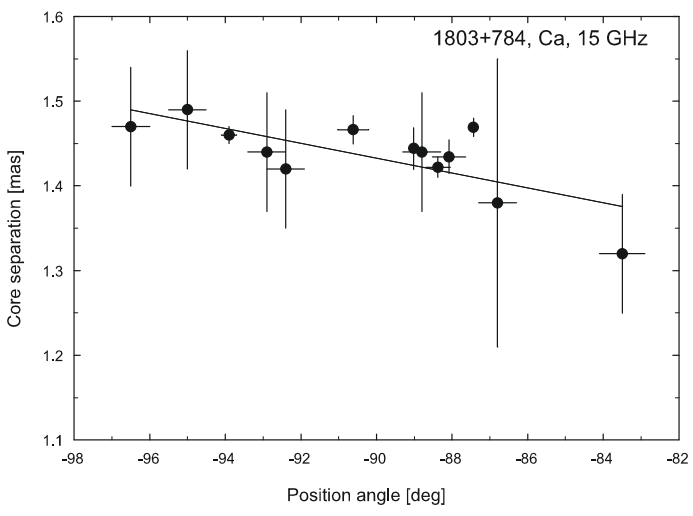

(a)

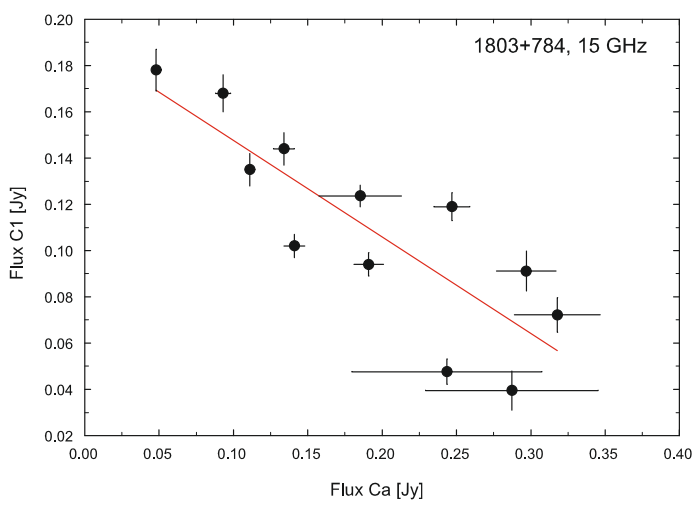

(b)

Fig. 13. Panel a) shows core separation as a function of position angle for the brightest component $\mathrm{Ca}$. Panel b) shows the flux density of component $\mathrm{C} 1$ against that of component $\mathrm{Ca}$. Both panels plot the parameters estimated from $15 \mathrm{GHz}$ observations from all the different epochs.

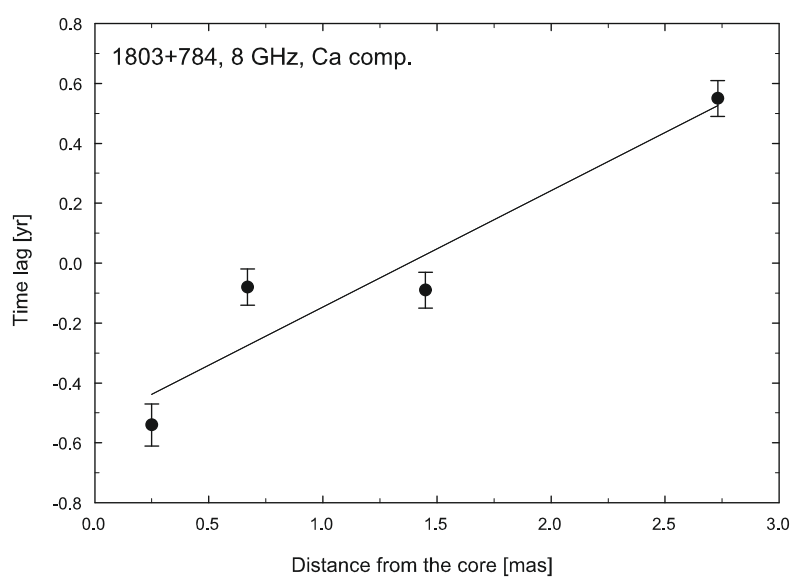

Fig. 14. Time lag of the discrete cross-correlation function of the core separation of component $\mathrm{Ca}$ vs. those of components $\mathrm{C} 0, \mathrm{C} 1, \mathrm{C} 2_{1}$, and $\mathrm{C}_{3}$ as a function of the mean value of distance from the core for these components. The linear regression fit to these time lags is overplotted.

outward superluminal motion. Applying our component identification procedure to available data sets from the literature leads to a consistent kinematic picture. Although these components remain at roughly constant core separations on the long term, they do exhibit significant changes in their position angles. This results in an evolution of the jet ridge line from a straight line to 
a sinusoidal form with a characteristic time-scale of 8-9 years. A similar evolution of the jet ridge line has so far only been observed in one other BL Lac object: 0716+714 (Britzen et al., in prep.). Following such jet ridge-line evolution proceeds independently of any component-identification-scenario but requires a large number of epochs to be discovered and traced over several periods of evolution.

We find a number of correlations between the parameters of the core and those of individual components. The best case is seen for $\mathrm{C} 1$ where the total flux, the component flux, the core separation, and the position angle are obviously related. Many other pairs of correlations or anti-correlations are presented in this paper. The set of all these correlations and periodicities together argues in favor of a geometric origin. In the following subsections, we discuss possible scenarios to explain the observed evolution in the pc-scale jet of S5 $1803+784$ and the kinematic behavior of its components.

\subsection{Curved jet structures/precession?}

Curved jet structures have been observed in many different sources, both extragalactic (e.g., 1928+738, OJ 287, 3C 273, $3 \mathrm{C} 449$ ) and Galactic (e.g. SS433, HH111). There are prominent differences between stellar and AGN jets, including the role of magnetic fields in the collimation and dynamical evolution of their structure and the jet-to-environment density ratio that is significantly larger for the stellar jets. Based on the later characteristic, the dynamical evolution of a stellar jet can more easily be treated ballistically, whereas such an assumption would not necessarily apply to the case of AGN jets (Hardee et al. 1994).

Curving and wiggling of jets is usually attributed to precession of the base of the jet. Two different scenarios concerning the central engine that feeds the jets can explain the observed curvature.

\subsubsection{Models relying on a single central Black Hole}

In the first scenario, the core that ejects the jet is considered to be a single object (usually a supermassive black hole, SMBH). Precession can then occur due to the Bardeen-Petterson effect through Lense-Thirring precession of the accretion disk (Caproni et al. 2006). However, this model assumes a rigid body configuration between the accretion disk and the jet, an assumption that we are not able to test or verify. A magnetic torque mechanism has also been proposed (Lai 2003), driven by the large-scale magnetic field threading through the accretion disk, leading to precession of the disk and consequently of the jet. In the same frame, a group of models attributes the curving and wiggling of the jet to magnetohydrodynamic (MHD) instabilities, most prominently Kelvin-Helmholtz (K-H) instabilities (Camenzind \& Krockenberger 1992; Hardee \& Norman 1988; Birkinshaw 1991; Zhao et al. 1992; Hardee et al. 1994, 1997; Meier \& Nakamura 2006; Perucho et al. 2006). Meier \& Nakamura (2006) consider the instability to be driven by current, related to the strong-field pinch, rather than by K-H instabilities. All models mentioned above strongly depend on the jetto-environment density ratio. However, instabilities have to be excited in order to affect the structure of the jet. Thus, although the MHD instabilities (and mainly the helical K-H kink instability) can possibly explain the structure of $\mathrm{pc}$ and kpc scale jets, the problem of how these modes are excited still remains.

\subsubsection{Models relying on a Binary Black Hole}

In the second scenario, models have been built under the assumption that a binary system can be found in the centers of AGN. The most popular idea concerns binary black hole systems $(\mathrm{BBH})$ as a product of galaxy merging. The problem is complex and each of the models makes a number of assumptions to explain the observed structures. In such systems, the primary black hole is considered to have an accretion disk and jets, whereas the secondary black hole is in orbit around the primary. Gravitational perturbations associated with this orbiting secondary black hole can disrupt the accretion disk of the primary black hole and lead to precession. Lobanov \& Roland (2005) argue that in light of the "two-fluid" model, the orbital motion and the disk precession can lead to curving and wiggling of the jet. Furthermore, they propose that for strong enough magnetic fields, the K-H instabilities can be considered negligible. Roos et al. (1993) argue that the orbital motion of the binary is responsible for the change of the jet-ejection direction and thus leads to a wiggling structure (the jet is treated ballistically). Kaastra \& Roos (1991) argue that the precession angle is determined by the angle between the primary black hole spin axis and the angular momentum of the binary system. As before, the jet is treated ballistically. Similar models have been proposed by Katz (1997) (a gravitational torque induced by the secondary black hole onto the accretion disk) and Romero et al. (2000) (nearrigid body configuration).

Roland et al. (2008) apply a BBH model to the data for S5 $1803+784$ presented and discussed in this paper. The wiggling of the jet is attributed to a precession of the accretion disk, the orbital motion of the binary, and the movement of the whole system around the center of gravity of the AGN host (the "twofluid" model is used again).

\subsubsection{Garden hose models}

Another group of models, usually identified as "garden-hose" models, have been extensively used for stellar jets. They assume a ballistic evolution of the jet, and place specific conditions on the distribution of the density of the jet and surrounding medium. Most prominent examples for this type of model are Hjellming \& Johnston (1981) (a kinematic model for SS433 X-ray binary), Raga et al. (1993) (an analytical model for the expansion of jets that get ejected with a time-dependent direction), Biro et al. (1995) (a 2D numerical simulation based on the analytical model of Raga et al. 1993, considering adiabatic and non-adiabatic expansion of the jet), Cliffe et al. (1996) (a 3D numerical simulation for precessing stellar jets considering interaction with the surrounding medium), and Stirling et al. (2002). Along the lines of Hjellming \& Johnston (1981), Gower et al. (1982) produced a similar model for radio AGN jets, taking into account projection, relativistic, and cosmological redshift effects. This family of models again does not answer the problem of how the original precession arises, but they do offer a plausible explanation of the evolution of such perturbed jets. As all these models assume ballistic behavior, it is possible that they might not describe AGN jets sufficiently well.

Rieger (2004) presented a comparison of different configurations for curved jets, explained by ballistic and nonballistic models. He proposes that a non-ballistic description of AGN jets is more appropriate. Geodesic (Lens-Thirring) precession of the accretion disk (Begelman et al. 1980) would produce a period much longer than observed in the light-curves of AGN (e.g. OJ 287, S5 1803+784). Instead those authors favor 
non-relativistic precession which leads to significantly smaller periods. Finally, the complete absence of magnetic fields and their effects on the evolution to the jet is another reason to doubt the applicability of the above "garden-hose" models in their present form to modeling of AGN jets. A model that would take into account MHD instabilities and the role of large-scale magnetic fields on the collimation and evolution of AGN jets could provide a good explanation of the observed structure in S5 $1803+784$. We are currently in the process of preparing a model in this direction and will apply it to the data for S5 $1803+784$ presented in this paper. This model shall explain the position angle changes as well as the secondary "oscillatory" motion of the individual jet components.

\subsubsection{External causes of jet curvature}

Finally we mention another group of models that rely on external causes for explanation of the curving and wiggling of jets. The bending of the jet could be attributed to interaction with the surrounding medium. This however can not fully explain the case of S5 $1803+784$, since such periodic and fine structure within the jet would imply an equally finely structured surrounding medium, for which there is no evidence. Blandford \& Icke (1978) attributed the structure of the jet of 3C 31 to dynamical interaction with a nearby galaxy (again treating the jet ballistically). Jägers \& de Grijp (1985), based on the model of Blandford \& Icke (1978), proposed a similar model for 3C 10, while studying the movement of discrete blobs along the jet. Lupton \& Gott (1984), proposed that the curving of the jet of 3C 449 is due to the orbital motion of the the central engine around the host's center of gravity. But likewise, the periodic and finely structured curvature of the S5 $1803+784$ jet would seem to preclude such longer-term motions as the full explanation.

Yokosawa \& Inoue (1985) combine some of the above mod-

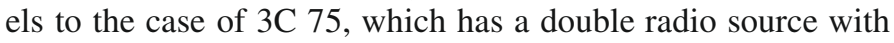
two pair of jets that seem to intertwine. Again treating the jets ballistically, the authors take into account the pressure of the surrounding medium, assume a time-dependent ejection velocity for the jets, and account for the orbital motion of the central engines of the jets.

A viable model should offer a self-consistent description and physical basis for the observed structure and behavior. It is important to include the accretion disk as well as the jet into the model. Moreover, in the case of S5 1803+784, the observed behavior in which most jet components remain at roughly constant core separations should also be explained. In this light, a twisting jet model can connect the observed structure and evolution of the jet to the base of the jet and the accretion disk itself. We follow Steffen et al. (1995) in describing an expanding synchrotron blob ejected from a rotating hot spot in an accretion disk around a supermassive black hole. This model has been introduced to capture the kinematic aspects of the jet model of Camenzind \& Krockenberger (1992) in the case of the apparent superluminal motion of jet components in 3C 345. In our scenario the jet is generated by collimated, relativistic outflow from an accretion disk, which is assumed to be in Keplerian rotation. Although our knowledge of the nozzle region between the disk and jet is not at all concrete, we can nonetheless make some viable assumptions that lead to a fairly good fit of the model to the data available. We are currently in the process of applying a twisted jet model to the data presented in this paper. Further studies on how such a hot spot in the accretion disk could come into being are however needed.

\subsection{Jet components at constant core separation}

As illustrated by the kinematic results for S5 $1803+784$ presented here, components that show no significant apparent superluminal (outward) motion over long time-scales can exist along the jet. Such components are also observed in a growing number of AGN (see e.g., Jorstad et al. 2001; Gómez et al.; Britzen et al. 2007). Different physical models and scenarios have been invoked to explain this phenomenon. A prominent example is the superluminal radio source 4C 39.25, in which a stationary component was observed by Alberdi et al. (2000). They explain this in geometrical terms, proposing that the stationary component is at a $90^{\circ}$ bend in the jet away from the observer. They disfavor the case of a recollimation shock based on polarization data. Gómez et al. (2001) find three quasi-stationary components in the jet of $0735+178$ in a quiescent state of the source, and attribute them to recollimation shocks and differential Doppler boosting. Britzen et al. (2009) show that $0735+178$ is a "hybrid" in the sense that the kinematic properties change simultaneously with morphological changes. At times when the pc-scale jet is straight, the jet components appear to be stationary. At times when the pc-scale jet appears to be strongly bent, the jet components show apparent superluminal motion. Britzen et al. (2007) and Kellermann et al. (2004) find stationary components in their 6 and $2 \mathrm{~cm}$ AGN surveys. We also note the case of the quasar 3C 395 (Simon et al. 1988; Lara et al. 1994, 1999), whose jet exhibits a possible stationary component due to possible bending of the jet. Martí \& Müller (2003) model AGN jets and find that pressure mismatches exist between the jet and the surrounding medium. These lead to the production of reconfinement shocks and energy density enhancements downstream of these shocks, which in turn give rise to stationary radio knots. Numerical simulations by Agudo et al. (2001) of an expanding relativistic jet show that the ejection of a superluminal component into the jet can trigger instabilities that can give rise to stationary components (shocks) in the wake of the superluminal component, especially in the near vicinity of the core.

In the case of S5 1803+784 we observe components that show no long-term change in their core separations, but do have position angles that vary in what appears to be a (quasi-)periodic fashion. Correlations among circular Gaussian parameters such as flux density, position angle, and core separation argue in favor of a geometric origin for the observed phenomena. We conclude that the kinematics in S5 1803+784 can not be explained easily with recourse to published models. With curved jets, there will be some cases where the jet crosses the observer's line of sight, creating inward and stationary features. However, it is difficult to apply such a scenario here, in which a number of components show roughly constant core separations and varying position angles. As mentioned in the previous sub-section, we are currently working on a jet model to explain these characteristics of S5 $1803+784$.

Acknowledgements. We thank the anonymous referee for helpful comments and suggestions. We are especially grateful to K. I. Kellermann, M. A. Pérez-Torres, A. Alberdi and J. M. Marcaide for sharing data, in part prior to publication. N. A. Kudryavtseva and M. Karouzos were supported for this research through a stipend from the International Max Planck Research School (IMPRS) for Radio and Infrared Astronomy. This research has made use of data from the MOJAVE database that is maintained by the MOJAVE team (Lister et al. 2009, AJ, 137, 3718). This research has made use of data from the University of Michigan Radio Astronomy Observatory which is supported by the National Science Foundation and by funds from the University of Michigan. S. Britzen acknowledges support by the Claussen-Simon-Stiftung. This research has made use of the NASA/IPAC Extragalactic Database (NED) which is operated by the Jet Propulsion Laboratory, California Institute of Technology, under contract with the National Aeronautics and Space Administration. The National Radio 
Astronomy Observatory is a facility of the National Science Foundation operated under cooperative agreement by Associated Universities, Inc. The European VLBI Network is a joint facility of European, Chinese, South African and other radio astronomy institutes funded by their national research councils. Based on observations with the 100-m telescope of the MPIfR (Max-Planck-Institut für Radioastronomie) at Effelsberg.

\section{References}

Agudo, I., Gómez, J. L., Martí, J. M., et al. 2001, ApJ, 549, 183

Alberdi, A., Gómez, J.-L., Marcaide, J. M., et al. 2000, A\&A, 361, 529

Aller, M. F., Aller, H. D., Hughes, P. A., \& Latimer, G. E. 1999, ApJ, 512, 601

Aller, M. F., Aller, H. D., \& Hughes, P. A. 2003, ApJ, 586, 33

Antonucci, R. R. J., Hickson, P., Olszewski, E. W., \& Miller, J. S. 1986, AJ, 92, 1

Appl, S., Sol, H., \& Vicente, L. 1996, A\&A, 310, 419

Biro, S., Raga, A. C., \& Cantó, J. 1995, MNRAS, 275, 557

Begelman, M. C., Blandford, R. D., \& Rees, M. J. 1980, Nature, 287, 307

Birkinshaw, M. 1991, MNRAS, 252, 505

Blandford, R. D., \& Icke, V. 1978, MNRAS, 185, 527

Britzen, S. 2002, Rev. Mod. Astron., 15, 199

Britzen, S., \& Krichbaum, T. P. 1995, in Proceedings of the 10th Working meeting on European VLBI for Geodesy and Astrometry, Centro Di Geodesia Spaziale, Matera, Italy, ed. R. Lanotte, \& G. Bianco, 172

Britzen, S., Witzel, A., Krichbaum, T. P., et al. 2005a, MNRAS, 362, 966

Britzen, S., Krichbaum, T. P., Strom, R. G., et al. 2005b, A\&A, 444, 443

Britzen, S., Meyer, V., Witzel, A., Agudo, I., et al. 2006, in Conf. proceedings of the 8th EVN Symposium, Torun, Poland, 6

Britzen, S., Vermeulen, R. C., Campbell, R. M., et al. 2007, A\&A, 472, 763

Britzen, S., Vermeulen, R. C., Campbell, R. M., et al. 2008, A\&A, 484, 119

Britzen, S., Witzel, A., Gong, B. P., et al. 2010, A\&A, in press, [arXiv: 1002.3531$]$

Camenzind, M., \& Krockenberger, M. 1992, A\&A, 255, 59

Caproni, A., Abraham, Z., \& Mosquera Cuesta, H. J. 2006, ApJ, 638, 120

Cawthorne, T. V., Wardle, J. F. C., Roberts, D. H., et al. 1993, ApJ, 416, 496

Cliffe, J. A., Frank, A., \& Jones, J. W. 1996, MNRAS, 282, 1114

Eckart, A., Witzel, A., Biermann, P., et al. 1986, A\&A, 168, 17

Eckart, A., Witzel, A., \& Biermann, P. 1987, A\&A, 67, 121

Edelson, R. A., \& Krolik, J. H. 1988, ApJ, 333, 646

Ferraz-Mello, S. 1981, AJ, 86, 619

Fey, A. L., Clegg, A. W., \& Fomalont, E. B. 1996, ApJS, 105, 299

Fomalont, E. B. 1999, in Synthesis Imaging in Radio Astronomy II, ed. G. B.

Taylor, C. L. Carilli, \& R. A. Perley, ASP Conf. Ser., 180, 301

Gabuzda, D. C., \& Chernetskii, V. A. 2003, MNRAS, 339, 669

Gabuzda, D. C., Cawthorne, T. V., Roberts, D. H., \& Wardle, J. F. C. 1992, ApJ, 388,40

Gabuzda, D. C., Mullan, C. M., Cawthorne, T. V., Wardle, J. F. C., \& Roberts, D. H. 1994, ApJ, 435, 140

Gower, A. C., Gregory, P. C., Hutchings, J. B., \& Unruh, W. G. 1982, ApJ, 262, 478

Gómez, J. L., Martí, J. M., Marscher, A. P., Ibáñez, J. M., \& Alberdi, A. 1997, ApJ, 482, L33

Gómez, J. L., Guirado, J. C., Agudo, I., et al. 2001, MNRAS, 328, 873

Guirado, J. C., Ros, E., Jones, D. L., et al. 2001, A\&A, 371, 766

Hardee, P. E., \& Norman, M. L. 1988, ApJ, 334, 70

Hardee, P. E., \& Stone, J. M. 1997, ApJ, 483, 121

Hardee, P. E., Cooper, M. A., \& Clarke, D. A. 1994, ApJ, 424, 126

Hardee, P. E., Clarke, D. A., \& Rosen, A. 1997, ApJ, 485, 533

Hjellming, R. M., \& Johnston, K. L. 1981, ApJ, 246, L141

Jägers, W. J., \& de Grijp, M. H. K. 1985, A\&A, 143, 176

Jorstad, S. G., Marscher, A. P., Lister, M. L., et al. 2005, AJ, 130, 1418

Jurkevich, I. 1971, AP\&SS, 13, 154

Kaastra, J. S., \& Roos, N. 1992, A\&A, 254, 96
Katz, J. I. 1997, ApJ, 478, 527

Kellermann, K. I., \& Pauliny-Toth, I. I. K. 1969, ApJ, 155, 71

Kellermann, K. I., Vermeulen, R. C., Zensus, J. A., \& Cohen, M. H. 1998, AJ, 115,1295

Kellermann, K. I., Lister, M. L., Homan, D. C., et al. 2004, ApJ, 609, 539

Kelly, B. C., Hughes, P. A., Aller, H. D., \& Aller, M. F. 2003, ApJ, 591, 695

Kidger, M., Takalo, L., \& Sillanpaa, A. 1992, A\&A, 264, 32

Krichbaum, T. P. 1990, in Parsec-scale Radio Jets (Cambridge: Cambridge University Press), ed. J. A. Zensus, \& T. J. Pearson, 83

Krichbaum, T. P., Witzel, A., Graham, D. A., et al. 1993, in Subarcsecond Radio Astronomy (Cambridge: Cambridge University Press), ed. R. J. Davies, \& R. S. Booth, 181

Kudryavtseva, N. A., Britzen, S., Witzel, A., et al. 2006, in Conf. proceedings of the 8th EVN Symposium, Torun, Poland, 7

Lai, D. 2003, ApJ, 591, L119

Lawrence, C. R., Readhead, A. C. S., Pearson, T. J., \& Unwin, S. C. 1987, in Superluminal Radio Sources (Cambridge: Cambridge University Press), ed. J. A. Zensus, \& T. J. Pearson, 260

Lister, M. L., \& Homan, D. C. 2005, AJ, 130, 1389

Lister, M. L., Tingay, S. J., Murphy, D. W., et al. 2001, ApJ, 554, 948

Lister, M. L., Cohen, M. H., Homan, D. C., et al. 2009, AJ, 138, 1874

Lobanov, A. P., \& Roland, J. 2005, A\&A, 431, 831

Lobanov, A. P., Krichbaum, T. P., \& Graham, D. A. 2000, A\&A, 364, 391

Lupton, R. H., \& Gott, III J. R. 1982, ApJ, 255, 408

Marcaide, J. M., Alberdi, A., Ros, E., et al. 1995, Nature, 373, 44

Marscher, A. P. 1996, in Blazar Variability, ed. H. R. Miller, J. R. Webb, \& J. C. Noble (San Francisco: ASP), ASP Conf. Ser., 110, 248

Marscher, A. P., \& Gear, W. K. 1985, ApJ, 298, 114

Martí, J. M., \& Müller, E. 2003, Living Reviews: http://relativity. livingreviews.org/Articles/lrr-2003-7/

Meier, D. L., \& Nakamura, M. 2006, ASP Conf. Ser., 350

Pérez-Torres, M. A., Marcaide, J. M., Guirado, J. C., et al. 2000, A\&A, 360, 161 Perucho, M., Lobanov, A. P., Martí, J. M., \& Hardee, P. E. 2006, A\&A, 456, 493

Piner, B. G., Mahmud, M., Fey, A. L., \& Gospodinova, K. 2007, AJ, 133, 2357

Pyatunina, T. B., Kudryavtseva, N. A., Gabuzda, D. C., et al. 2006, MNRAS, 373,1470

Pyatunina, T. B., Kudryavtseva, N. A., Gabuzda, D. C., et al. 2007, MNRAS, 381,797

Raga, A. C., Cantó, J., \& Biro, S. 1993, MNRAS, 260, 163

Rieger, F. M. 2004, ApJ, 615, L5

Roland, J., Britzen, S., Kudryavtseva, N. A., Witzel, A., \& Karouzos, M. 2008, A\&A, 483, 125

Romero, C. E., Chajet, L., Abraham, Z., \& Fan, J. H. 2000, A\&A, 360, 57

Roos, N. 1988, ApJ, 334, 95

Roos, N., Kaastra, J. S., \& Hummel, C. A. 1993, ApJ, 409, 130

Ros, E., Marcaide, J. M., Guirado, J. C., et al. 2000, A\&A, 356, 357

Ros, E., Marcaide, J. M., Guirado, J. C., \& Pérez-Torres, M. A. 2001, A\&A, 376, 1090

Schalinski, C. J., Alef, W., Witzel, A., et al. 1988, in Proc. IAU Symp. 129, ed. M. J. Reid, \& J. M. Moran (Dordrecht: Kluwer), 359

Shepherd, M. C. 1997, Astronomical Data Analysis Software and Systems VI, ed. G. Hunt, \& H. E. Payne, ASP Conf. Ser., 125, 77

Steffen, W., Zensus, J. A., Krichbaum, T. P., Witzel, A., \& Qian, S. J. 1995, A\&A, 302, 335

Stickel, M., Fried, J. W., \& Kühr, H. 1993, A\&AS, 98, 393

Stirling, A. M., Jowett, F. H., Spencer, R. E., et al. 2002, MNRAS, 337, 657

Tateyama, C. E., Kingham, K. A., Kaufmann, P., \& de Lucena, A. M. P. 2002, ApJ, 573, 496

Wagner, S. J., \& Witzel, A. 1995, ARA\&A, 33, 163

Witzel, A. 1987, in Superluminal Radio Sources, ed. J. A. Zensus, \& T. J. Pearson (Cambridge: Cambridge University Press), 83

Witzel, A., Schalinski, C. J., Johnston, K. J., et al. 1988, A\&A, 206, 245

Yokosawa, M., \& Inoue, M. 1985, Publ. Astron. Soc. Japan, 37, 655

Zhao, J. H., Burns, J. O., Norman, M. L., \& Sulkanen, M. E. 1992, ApJ, 387, 83

Pages 16 to 25 are available in the electronic edition of the journal at http: //www . aanda.org 


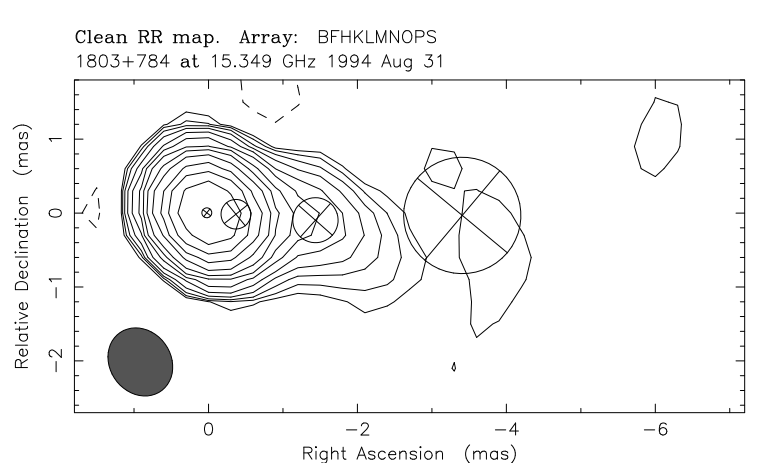

Map center: RA: 1800 45.684, Dec: $+782804.018(2000.0)$ Map peak: $1.18 \mathrm{Jy} /$ beam

Contours \%: $-0.65 \quad 0.65 \quad 0.975 \quad 1.462 .193 .294 .94$ Contours \%: 7.411 .116 .72537 .556 .2

(a)

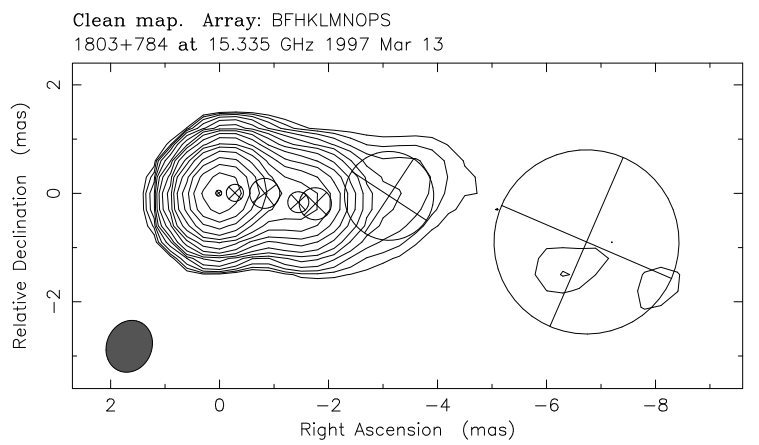

Map center: RA: 1800 45.684, Dec: +78 $2804.018(2000.0)$

Map peak: $1.56 \mathrm{Jy} /$ beam

Contours \%: 0.150 .2250 .3370 .5060 .7591 .141 .71

Contours \%: 2.56 3.845 .778 .651319 .529 .243 .8

(c)

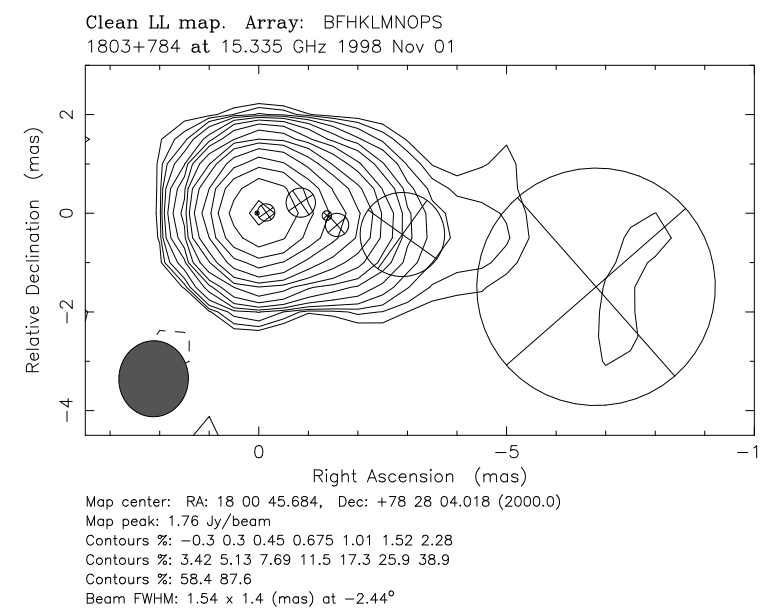

(e)

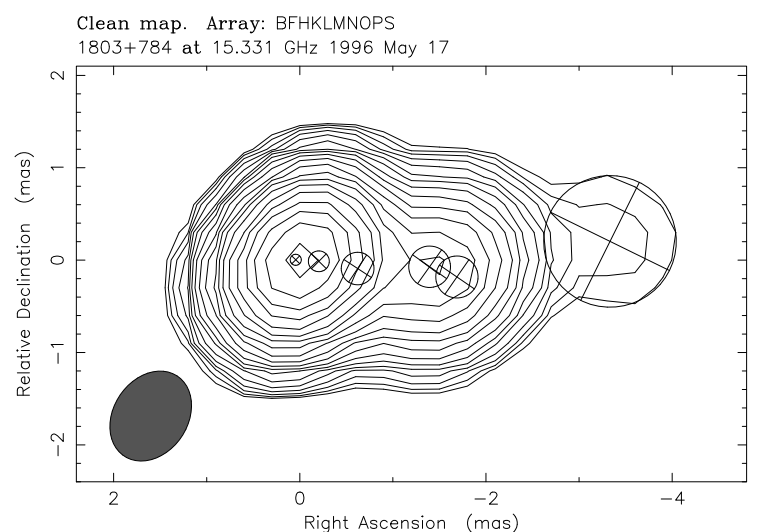

Map center: RA: 1800 45.684, Dec: +78 2804.018 (2000.0)

Map peak: $2.02 \mathrm{Jy} /$ beam

Contours \%: 0.20 .280 .3920 .5490 .7681 .081 .51

Contours \%: 31.143 .66185 .4

(b)

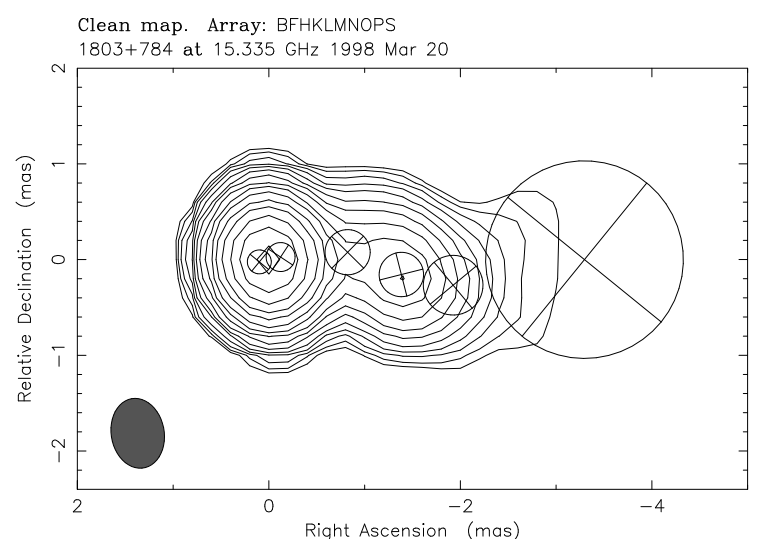

Wap center: PA: $1800-45084$, Dece $+782804.018(2000.0)$

Map peak: 1.55 Jy/beam

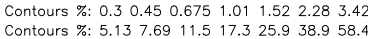

Contours \%: 87.

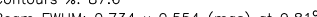

(d)

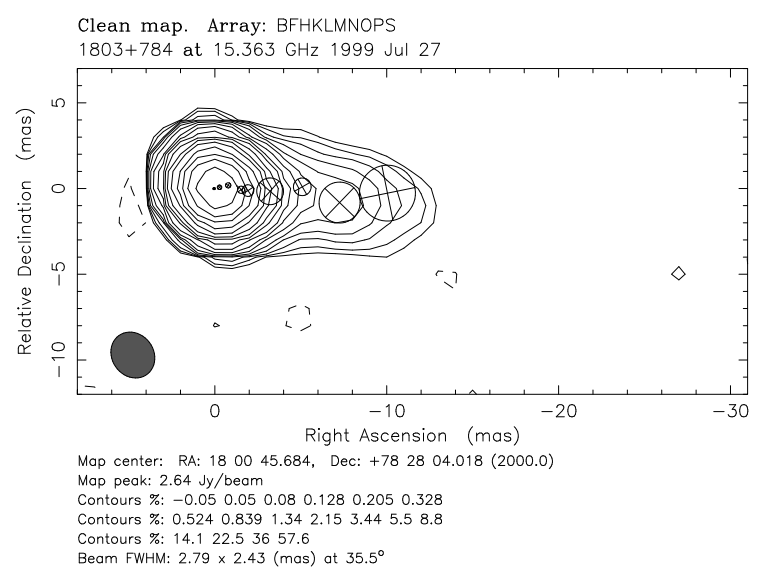

(f)

Fig. 15. Results of hybrid imaging (contours) and circular Gaussian model fitting (circles with crosses) for S5 $1803+784$ at $15 \mathrm{GHz}$. 
S. Britzen et al.: The kinematics in the pc-scale jets of AGN

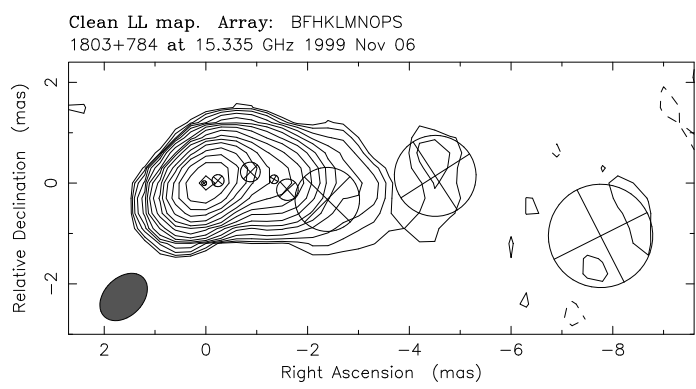

Map center: RA: 1800 45.684, Dec: +782804.018 (2000.0)

Map peak: 1.96 Jy/beam
Contours \%: -0.20 .20 .30 .450 .6751 .011 .522 .28
Contours \%: 3.425 .137 .6911 .517 .325 .938 .9

Contours $\%$

Beam FWHM: $1.09 \times 0.761$ (mas) at $-44.5^{\circ}$

(a)

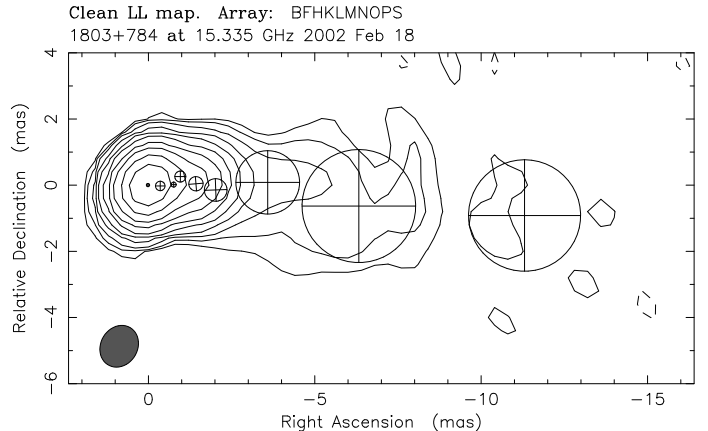

Map center: RA: 1800 45.684, Dec: +782804.019 (2000.0)

Map peak: $1.79 \mathrm{Jy} /$ beam

Contours \%: 25.651 .2

(1)

(c)

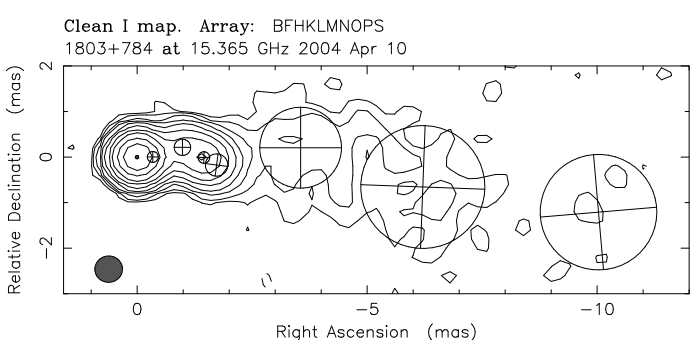

Map center: RA: 1800 45.684, Dec: +782804.019 (2000.0)

Map peak: $1.26 \mathrm{Jy} / \mathrm{beam}$

101.020 .40 .81 .63 .26 .412 .8

Beam FWHM: $0.6 \times 0.579$ (mas) at $-84.5^{\circ}$

(e)

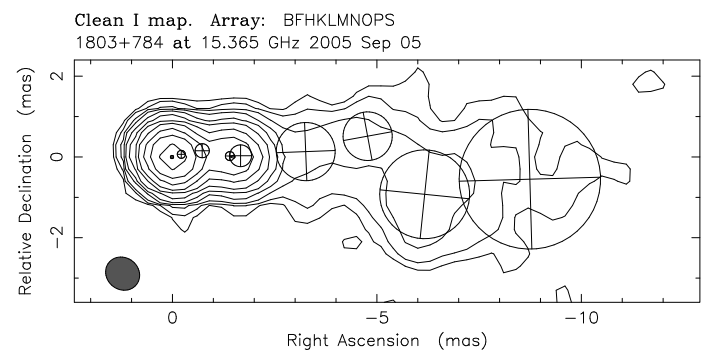

Wap center: RA: 180045.684, Doec +782804018 (2000

Map peak: $1.47 \mathrm{Jy} /$ beam

0.250 .521 .042 .084 .1

Con:ours \%: 0.3216 .633 .366 .6

(g)

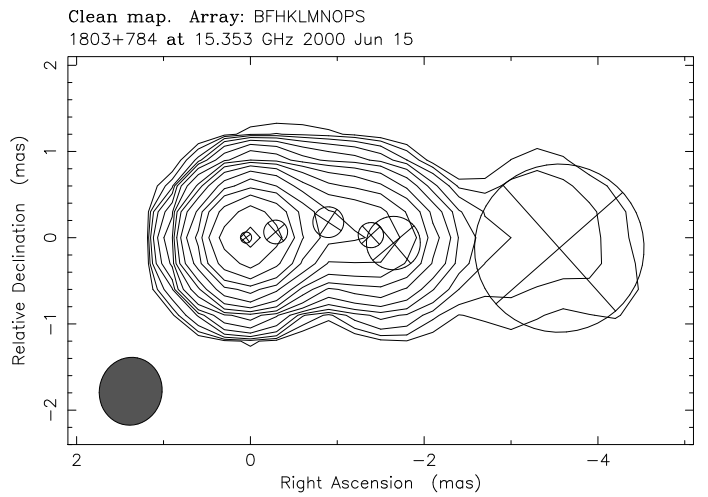

Map center: RA: 1800 45.684, Dec: +782804.018 (2000.0)

Map peak: $1.77 \mathrm{Jy} /$ beam
Contours \%: 0.20 .30 .450 .6751 .011 .522 .283 .42
Contours \%: 5.137 .6911 .517 .325 .938 .958 .4

Bearn FWHM: $0.79 \times 0.722$ (mas) at $-11.2^{\circ}$

(b)

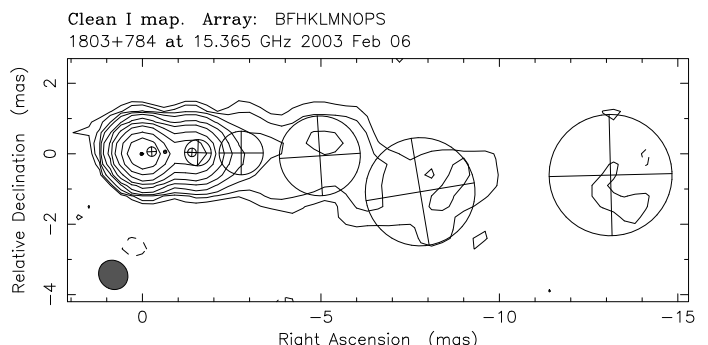

Map center: RA: 1800 45.684, Dec: 7782804.019 (2000.0)

Map peak: 1.41 Jy/beam

Contours \%. -0.0950 .0950 .190 .380 .761 .523 .04$

Ream FWHM: $0.866 \times 0.787$ (mas) at $41.3^{\circ}$

(d)

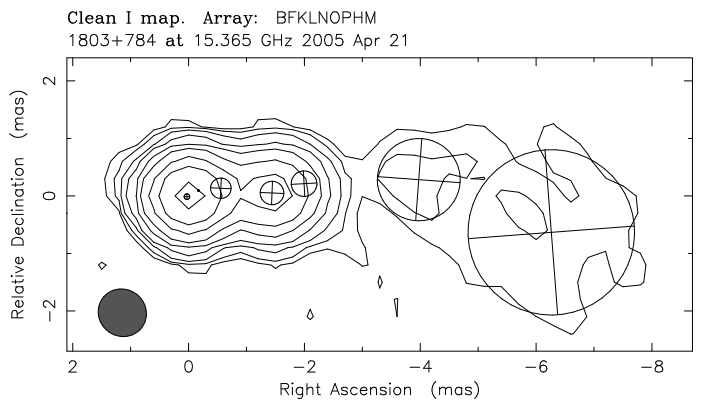

Map center: RA: 180045.684, Dec: $+782804.018(2000.0)$

Contours \%: 0.30 .67 .22 .44 .89 .619 .238 .476 .8

(f)

Fig. 16. Results of hybrid imaging (contours) and circular Gaussian model fitting (circles with crosses) for S5 $1803+784$ at $15 \mathrm{GHz}$; continuation of Fig. 15. 


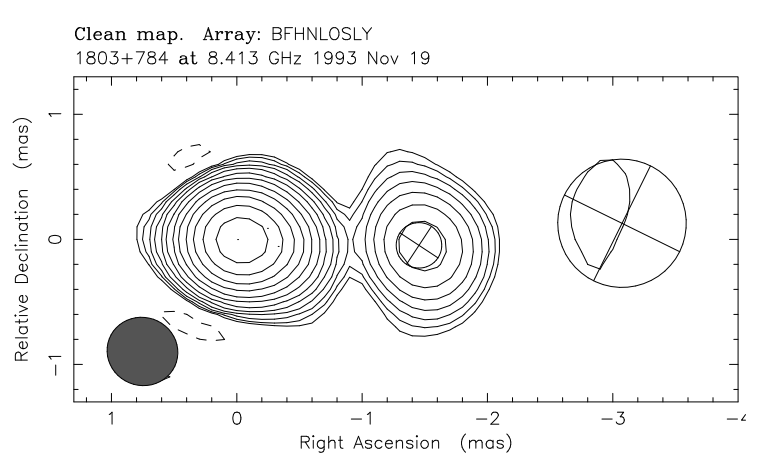

Map center: RA: 1803 39.201, Dec: +782754.299 (1950.0)

Map peak: $1.51 \mathrm{Jy} / \mathrm{beam}$

Contours \%: -0.850 .851 .281 .912 .874 .36 .45$

Contours \%: $9.6814 .521 .832 .74973 .5{ }^{\circ}$

(a)

Clean map. Array: BFHKLMNOPS

$1803+784$ at $8.419 \mathrm{GHz} 1997 \mathrm{Dec} 06$

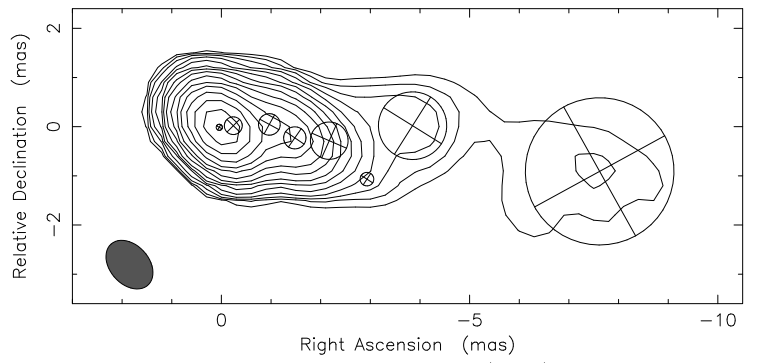

Map center: RA: 1800 45.684, Dec: +782804.018 (2000.0)

Map peak: $1.54 \mathrm{Jy} /$ beam

Contours \%: 0.150 .240 .3840 .6140 .9831 .572 .52

Contours \%: $4.036 .44 \quad 10.3 \quad 16.526 .442 .267 .6$

(c)

Clean RR map. Array: BFHKLMNOPS

$1803+784$ at $8.419 \mathrm{GHz} 2001 \mathrm{Feb} 04$

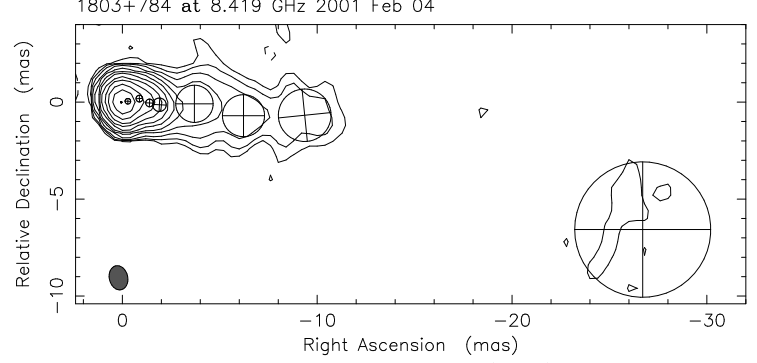

Map center: RA: 1800 45.684, Dec: +78 2804.018 (2000.0)

Map peak: $1.55 \mathrm{Jy} / \mathrm{beam}$

Contours \%: -0.050 .050 .10 .20 .40 .81 .63 .26 .4$

Beam FWHM: $1.26 \times 0.9$

(e)

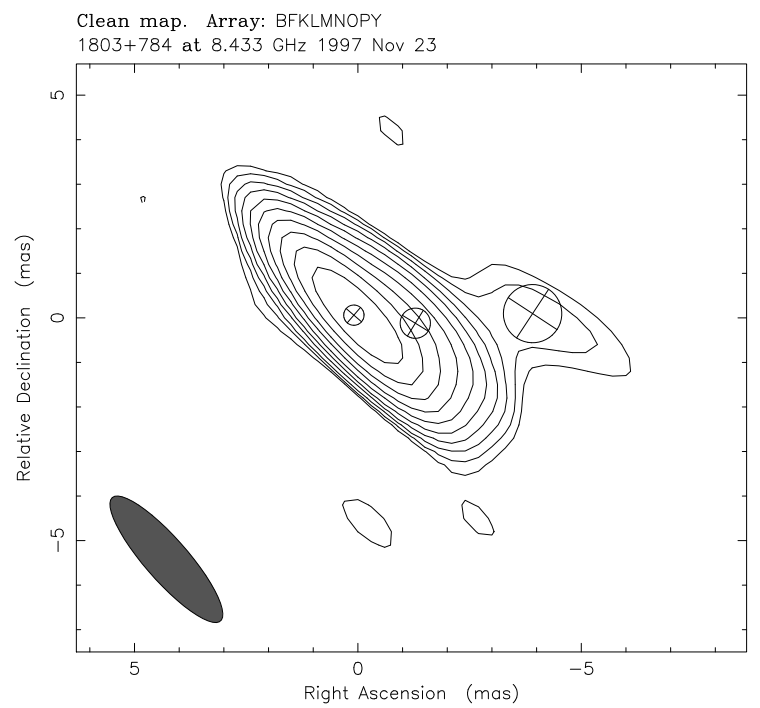

Map center: RA: 1800 45.684, Deci +78 2804018 (2000)

Map peak: $2.34 \mathrm{Jy} /$ bear

Contours \%: -1.711 .72 .553 .835 .748 .6112 .919 .4$

(b)

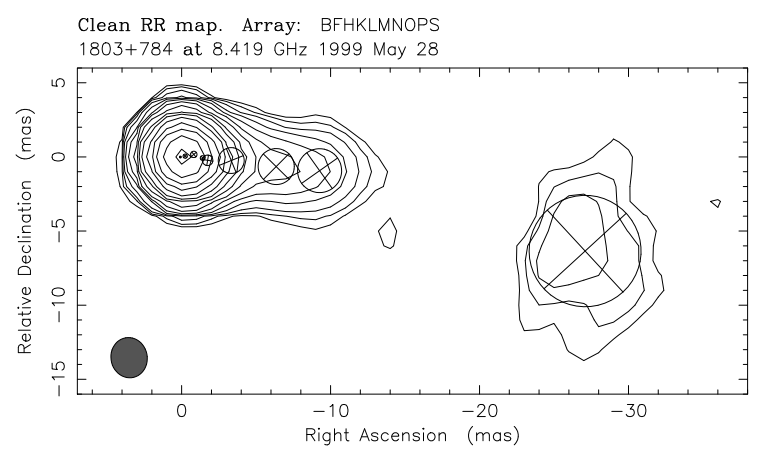

Map center: RA: 1800 45.684, Dec: +78 2804.018 (2000.0)

Map peak: $1.94 \mathrm{Jy} / \mathrm{beam}$

Contours \%: $0.050 .0850 .145 \quad 0.2460 .418 \quad 0.711 .21$

Contours \%: 2.053 .14

Beam FWHM: $2.72 \times 2.44$ (mas) at $9.71^{\circ}$

(d)

Fig. 17. Results of hybrid imaging (contours) and circular Gaussian model fitting (circles with crosses) for S5 $1803+784$ at 8.4 GHz. 
S. Britzen et al.: The kinematics in the pc-scale jets of AGN

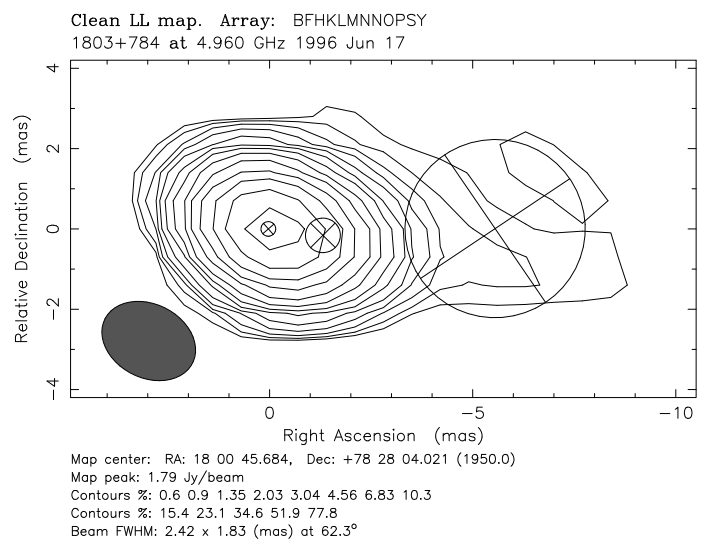

(a)

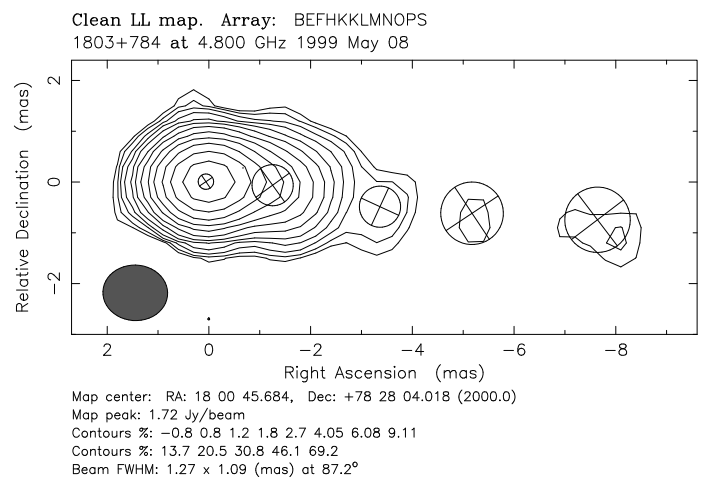

(c)

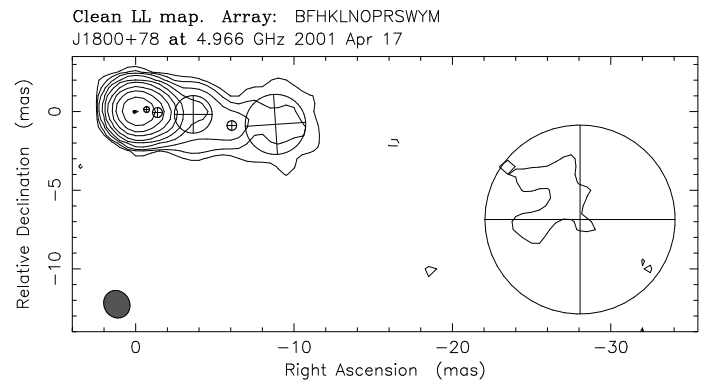

Map center: RA: 1800 45.684, Dec: +78 2804.018 (2000.0)

Wap peak: $1.77 \mathrm{Jy} / \mathrm{beam}$

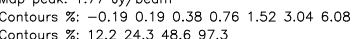

Beam FWHM: $1.8 \times 1.62$ (mas) at $309^{\circ}$

(e)

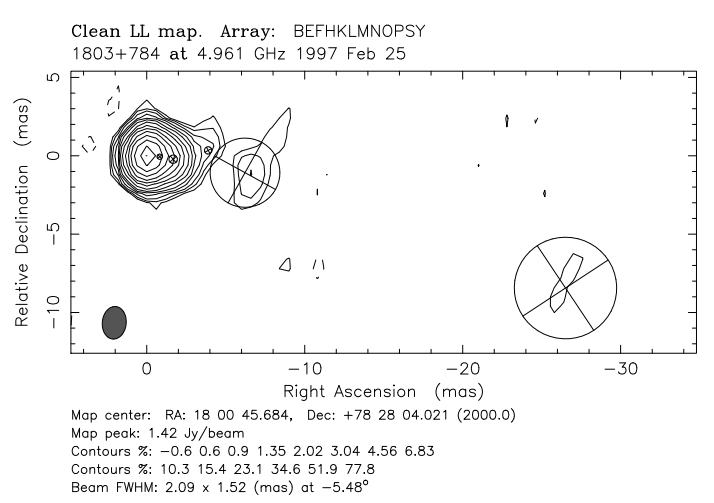

(b)

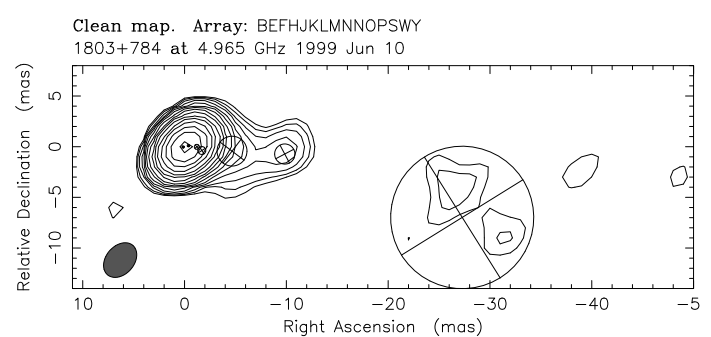

Map center: RA: 1800 45.684, Dec: 7782804018 (2000.0)

Map peak: $2.08 \mathrm{Jy} / \mathrm{beam}$

Contours \%: 0.30 .450 .6751 .011 .522 .283 .42

Contours \%: 87.6
Beam FWHM: $3.83 \times 2.78$ (mas) at $-40.6^{\circ}$

(d)

Fig. 18. Results of hybrid imaging (contours) and circular Gaussian model fitting (circles with crosses) for S5 $1803+784$ at $5 \mathrm{GHz}$.

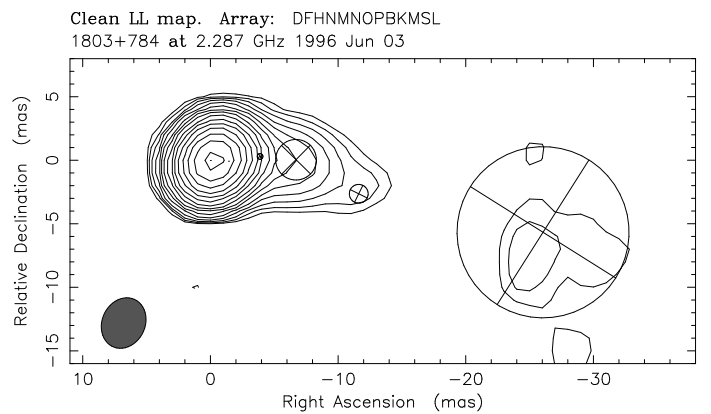

Map center: RA: 1803 39.167. Dec: $+782754.300(1950.0)$

Wap peak: $1.93 \mathrm{Jy} /$ beam

Contours \%: -0.80 .81 .121 .572 .23 .074 .36 .02$

Ceam FWHM: $4.09 \times 3.37$ (mas) at $-24.4^{\circ}$

Fig. 19. Results of hybrid imaging (contours) and circular Gaussian model fitting (circles with crosses) for S5 $1803+784$ at $2.3 \mathrm{GHz}$. 
A\&A 511, A57 (2010)

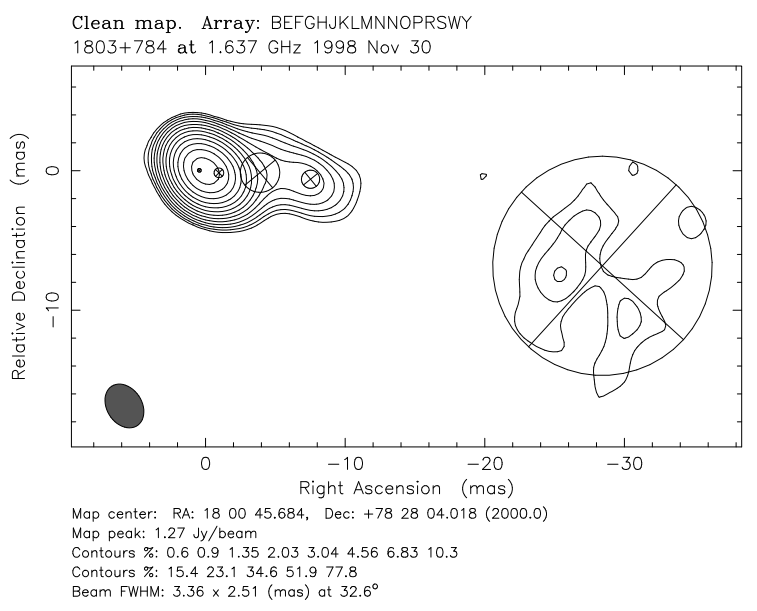

(a)

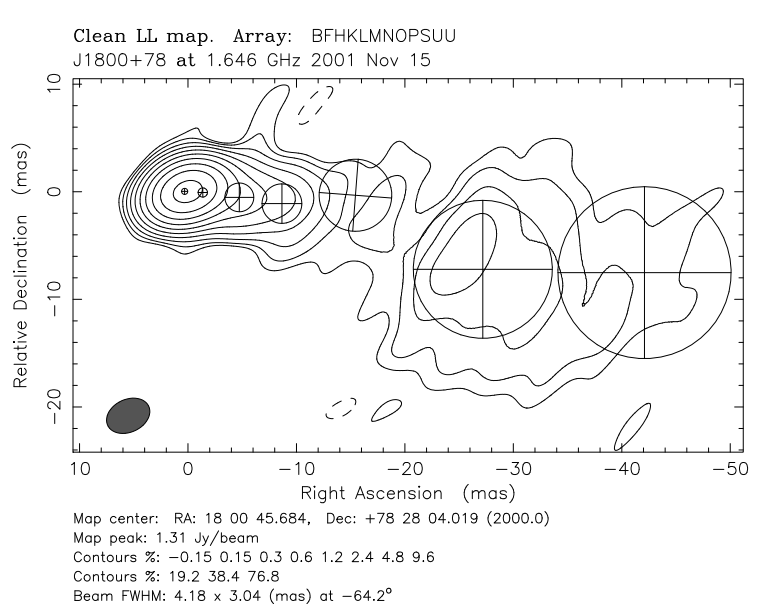

(b)

Fig. 20. Results of hybrid imaging (contours) and circular Gaussian model fitting (circles with crosses) for S5 $1803+784$ at $1.6 \mathrm{GHz}$.

Table 1. Circular Gaussian model-fit results for S5 1803+784. The columns list the epoch of observation, the jet-component identification, the flux density, the radial distance of the component center from the core, the position angle of the center of the component, the FWHM major axis, and the chi-square of the fit.

\begin{tabular}{|c|c|c|c|c|c|c|}
\hline $\begin{array}{c}(1) \\
\text { Epoch }\end{array}$ & $\begin{array}{l}\text { (2) } \\
\text { Id. }\end{array}$ & $\begin{array}{r}(3) \\
S \text { [Jy] } \\
\end{array}$ & $\begin{array}{r}(4) \\
\mathrm{r} \text { [mas] }\end{array}$ & $\begin{array}{r}(5) \\
\theta[\mathrm{deg}] \\
\end{array}$ & $\begin{array}{r}(6) \\
\text { M.A. [mas] }\end{array}$ & $\begin{array}{l}(7) \\
\chi^{2}\end{array}$ \\
\hline & & & $1.6 \mathrm{GHz}$ & & & \\
\hline 1998.92 & $\mathrm{r}$ & $0.946 \pm 0.142$ & 0.00 & 0.0 & $0.26 \pm 0.02$ & 38.232803 \\
\hline 1998.92 & $\mathrm{Ca}$ & $0.558 \pm 0.084$ & $1.41 \pm 0.05$ & $262.7 \pm 0.8$ & $0.69 \pm 0.03$ & \\
\hline 1998.92 & $\mathrm{C} 4$ & $0.091 \pm 0.014$ & $4.35 \pm 0.44$ & $267.8 \pm 1.0$ & $2.82 \pm 0.30$ & \\
\hline 1998.92 & $\mathrm{C} 8$ & $0.059 \pm 0.009$ & $7.99 \pm 0.80$ & $265.4 \pm 2.0$ & $1.30 \pm 0.35$ & \\
\hline 1998.92 & C30 & $0.259 \pm 0.052$ & $29.67 \pm 3.00$ & $256.7 \pm 4.0$ & $15.72 \pm 4.00$ & \\
\hline 2001.87 & $\mathrm{r}$ & $1.233 \pm 0.169$ & $0.00 \pm 0.01$ & $0.0 \pm 0.5$ & $0.60 \pm 0.08$ & 0.0923 \\
\hline 2001.87 & $\mathrm{Ca}$ & $0.266 \pm 0.047$ & $2.33 \pm 0.71$ & $-94.8 \pm 1.5$ & $1.12 \pm 0.29$ & \\
\hline 2001.87 & $\mathrm{C} 8$ & $0.134 \pm 0.019$ & $6.15 \pm 0.98$ & $-96.7 \pm 1.2$ & $3.17 \pm 0.57$ & \\
\hline 2001.87 & $\mathrm{C} 12$ & $0.058 \pm 0.010$ & $9.45 \pm 0.42$ & $-97.0 \pm 0.9$ & $3.70 \pm 0.11$ & \\
\hline 2001.87 & & $0.010 \pm 0.001$ & $16.85 \pm 1.10$ & $-91.4 \pm 0.9$ & $5.10 \pm 1.60$ & \\
\hline 2001.87 & C30 & $0.241 \pm 0.034$ & $28.94 \pm 0.49$ & $-104.7 \pm 1.0$ & $14.18 \pm 0.79$ & \\
\hline \multirow{2}{*}{2001.87} & & $0.045 \pm 0.007$ & $43.07 \pm 0.61$ & $-100.0 \pm 0.8$ & $15.98 \pm 3.20$ & \\
\hline & & & $2.3 \mathrm{GHz}$ & & & \\
\hline 1996.42 & $\mathrm{r}$ & $1.642 \pm 0.246$ & 0.00 & 0.0 & $0.00 \pm 0.01$ & 86.988178 \\
\hline 1996.42 & $\mathrm{Ca}$ & $0.481 \pm 0.072$ & $1.43 \pm 0.07$ & $-92.2 \pm 0.5$ & $0.01 \pm 0.01$ & \\
\hline 1996.42 & $\mathrm{C} 4$ & $0.041 \pm 0.006$ & $3.91 \pm 0.39$ & $-85.3 \pm 1.0$ & $0.44 \pm 0.02$ & \\
\hline 1996.42 & $\mathrm{C} 8$ & $0.124 \pm 0.025$ & $6.71 \pm 0.80$ & $-89.4 \pm 1.8$ & $3.19 \pm 0.32$ & \\
\hline 1996.42 & $\mathrm{C} 12$ & $0.028 \pm 0.010$ & $11.92 \pm 2.38$ & $-102.7 \pm 1.9$ & $1.47 \pm 0.15$ & \\
\hline \multirow[t]{2}{*}{1996.42} & $\mathrm{C} 30$ & $0.204 \pm 0.069$ & $26.67 \pm 3.00$ & $-102.2 \pm 3.9$ & $13.47 \pm 2.70$ & \\
\hline & & & $5.0 \mathrm{GHz}$ & & & \\
\hline 1996.46 & $\mathrm{r}$ & $1.673 \pm 0.251$ & 0.00 & 0.0 & $0.36 \pm 0.02$ & 52.027293 \\
\hline 1996.46 & $\mathrm{Ca}$ & $0.422 \pm 0.063$ & $1.35 \pm 0.07$ & $-96.8 \pm 1.1$ & $0.86 \pm 0.04$ & \\
\hline 1996.46 & & $0.090 \pm 0.014$ & $5.58 \pm 0.60$ & $-89.9 \pm 1.54$ & $4.44 \pm 0.44$ & \\
\hline 1997.15 & $\mathrm{r}$ & $1.302 \pm 0.195$ & 0.00 & 0.0 & $0.01 \pm 0.01$ & 27.458208 \\
\hline 1997.15 & $\mathrm{C} 1$ & $0.274 \pm 0.041$ & $0.87 \pm 0.04$ & $-94.2 \pm 0.9$ & $0.33 \pm 0.07$ & \\
\hline 1996.15 & $\mathrm{Ca}$ & $0.218 \pm 0.033$ & $1.73 \pm 0.09$ & $-97.3 \pm 0.7$ & $0.53 \pm 0.08$ & \\
\hline 1997.15 & $\mathrm{C} 4$ & $0.017 \pm 0.002$ & $3.95 \pm 0.40$ & $-85.0 \pm 1.4$ & $0.47 \pm 0.10$ & \\
\hline 1997.15 & $\mathrm{C} 8$ & $0.081 \pm 0.016$ & $6.36 \pm 0.66$ & $-99.7 \pm 1.9$ & $4.42 \pm 0.44$ & \\
\hline 1997.15 & C30 & $0.067 \pm 0.013$ & $27.87 \pm 2.81$ & $-107.6 \pm 3.9$ & $6.48 \pm 0.84$ & \\
\hline 1999.35 & $\mathrm{r}$ & $1.774 \pm 0.266$ & 0.00 & 0.00 & $0.30 \pm 0.02$ & 15.738181 \\
\hline 1999.35 & $\mathrm{C} 1$ & $0.033 \pm 0.005$ & $0.77 \pm 0.04$ & $-69.4 \pm 0.5$ & $0.00 \pm$ & \\
\hline 1999.35 & $\mathrm{Ca}$ & $0.507 \pm 0.189$ & $1.31 \pm 0.07$ & $-93.0 \pm 1.5$ & $0.81 \pm 0.12$ & \\
\hline 1999.35 & $\mathrm{C} 4$ & $0.031 \pm 0.025$ & $3.46 \pm 0.35$ & $-98.1 \pm 1.1$ & $0.81 \pm 0.41$ & \\
\hline 1999.44 & $\mathrm{r}$ & $1.423 \pm 0.213$ & 0.00 & 0.0 & $0.21 \pm 0.01$ & \\
\hline 1999.44 & $\mathrm{C} 1$ & $0.450 \pm 0.067$ & $0.53 \pm 0.03$ & $-75.7 \pm 1.0$ & $0.22 \pm 0.01$ & \\
\hline 1999.44 & $\mathrm{Ca}$ & $0.274 \pm 0.041$ & $1.34 \pm 0.07$ & $-89.2 \pm 0.7$ & $0.49 \pm 0.03$ & \\
\hline 1999.44 & $\mathrm{C} 2$ & $0.101 \pm 0.015$ & $1.86 \pm 0.09$ & $-100.6 \pm 0.8$ & $0.78 \pm 0.08$ & \\
\hline 1999.44 & $\mathrm{C} 4$ & $0.071 \pm 0.011$ & $4.81 \pm 0.48$ & $-94.8 \pm 1.1$ & $2.94 \pm 0.30$ & \\
\hline 1999.44 & $\mathrm{C} 12$ & $0.033 \pm 0.006$ & $10.00 \pm 1.00$ & $-93.9 \pm 1.6$ & $1.99 \pm 0.20$ & \\
\hline 1999.44 & C30 & $0.099 \pm 0.020$ & $28.27 \pm 2.83$ & $-104.2 \pm 2.7$ & $14.06 \pm 2.58$ & \\
\hline
\end{tabular}


Table 1. continued.

\begin{tabular}{|c|c|c|c|c|c|c|}
\hline (1) & (2) & (3) & (4) & (5) & (6) & (7) \\
\hline Epoch & Id. & $\mathrm{S}[\mathrm{Jy}]$ & $\mathrm{r}[\mathrm{mas}]$ & $\theta$ [deg] & M.A. [mas] & $\chi^{2}$ \\
\hline 2001.29 & $\mathrm{r}$ & $1.602 \pm 0.246$ & $0.00 \pm 0.01$ & $0.0 \pm 0.5$ & $0.21 \pm 0.05$ & $\frac{\lambda}{0.0477}$ \\
\hline 2001.29 & $\mathrm{C} 1$ & $0.511 \pm 0.071$ & $0.85 \pm 0.08$ & $-81.1 \pm 2.1$ & $0.43 \pm 0.11$ & \\
\hline 2001.29 & $\mathrm{Ca}$ & $0.228 \pm 0.069$ & $1.62 \pm 0.38$ & $-91.7 \pm 3.5$ & $0.72 \pm 0.22$ & \\
\hline 2001.29 & $\mathrm{C} 4$ & $0.045 \pm 0.023$ & $3.89 \pm 0.18$ & $-84.7 \pm 4.9$ & $1.38 \pm 0.61$ & \\
\hline 2001.29 & $\mathrm{C} 8$ & $0.052 \pm 0.022$ & $6.07 \pm 0.24$ & $-98.3 \pm 0.8$ & $2.05 \pm 0.85$ & \\
\hline 2001.29 & $\mathrm{C} 12$ & $0.039 \pm 0.018$ & $9.23 \pm 0.35$ & $-95.1 \pm 1.7$ & $3.22 \pm 0.68$ & \\
\hline 2001.29 & $\mathrm{C} 30$ & $0.112 \pm 0.018$ & $29.12 \pm 0.59$ & $-103.3 \pm 1.2$ & $11.83 \pm 0.19$ & \\
\hline & & & $8.4 \mathrm{GHz}$ & & & \\
\hline 1993.88 & $\mathrm{r}$ & $1.390 \pm 0.208$ & 0.00 & 0.00 & $0.01 \pm$ & 2.8677791 \\
\hline 1993.88 & $\mathrm{CO}$ & $0.014 \pm 0.002$ & $0.25 \pm 0.01$ & $-69.3 \pm 0.7$ & $0.00 \pm 0.01$ & \\
\hline 1993.88 & $\mathrm{Cx}$ & $0.262 \pm 0.039$ & $0.33 \pm 0.02$ & $-99.5 \pm 0.4$ & $0.00 \pm 0.01$ & \\
\hline 1993.88 & $\mathrm{Ca}$ & $0.263 \pm 0.039$ & $1.45 \pm 0.07$ & $-91.8 \pm 0.8$ & $0.36 \pm 0.02$ & \\
\hline 1993.88 & $\mathrm{C} 4$ & $0.043 \pm 0.016$ & $3.07 \pm 0.11$ & $-87.5 \pm 1.2$ & $1.03 \pm 0.17$ & \\
\hline 1997.90 & $\mathrm{r}$ & $2.467 \pm 0.370$ & 0.00 & 0.00 & $0.45 \pm 0.02$ & 6.8563583 \\
\hline 1997.90 & $\mathrm{Ca}$ & $0.719 \pm 0.108$ & $1.39 \pm 0.07$ & $-97.4 \pm 1.3$ & $0.68 \pm 0.03$ & \\
\hline 1997.90 & $\mathrm{C} 4$ & $0.127 \pm 0.019$ & $4.00 \pm 0.45$ & $-89.5 \pm 1.3$ & $1.31 \pm 0.13$ & \\
\hline 1997.93 & $\mathrm{r}$ & $1.353 \pm 0.203$ & 0.00 & 0.00 & $0.12 \pm 0.01$ & 1.7453468 \\
\hline 1997.93 & $\mathrm{C} 0$ & $0.270 \pm 0.041$ & $0.28 \pm 0.01$ & $-83.5 \pm 1.9$ & $0.36 \pm 0.02$ & \\
\hline 1997.93 & $\mathrm{C} 1$ & $0.176 \pm 0.026$ & $1.01 \pm 0.05$ & $-86.6 \pm 0.6$ & $0.44 \pm 0.02$ & \\
\hline 1997.93 & $\mathrm{Ca}$ & $0.212 \pm 0.032$ & $1.54 \pm 0.08$ & $-98.0 \pm 0.5$ & $0.45 \pm 0.02$ & \\
\hline 1997.93 & $\mathrm{C} 2$ & $0.035 \pm 0.005$ & $2.23 \pm 0.11$ & $-97.0 \pm 1.0$ & $0.76 \pm 0.04$ & \\
\hline 1997.93 & $\mathrm{C} 4$ & $0.004 \pm 0.001$ & $3.16 \pm 0.32$ & $-109.5 \pm 1.0$ & $0.28 \pm 0.03$ & \\
\hline 1997.93 & $\mathrm{C} 4$ & $0.028 \pm 0.004$ & $3.89 \pm 0.39$ & $-89.5 \pm 1.0$ & $1.38 \pm 0.14$ & \\
\hline 1997.93 & $\mathrm{C} 8$ & $0.039 \pm 0.008$ & $7.71 \pm 0.90$ & $-96.6 \pm 3.0$ & $3.00 \pm 0.80$ & \\
\hline 1999.41 & $\mathrm{r}$ & $1.257 \pm 0.188$ & 0.00 & 0.0 & $0.12 \pm$ & 1.5628510 \\
\hline 1999.41 & $\mathrm{C} 0$ & $0.496 \pm 0.074$ & $0.33 \pm 0.02$ & $-79.7 \pm 1.1$ & $0.28 \pm 0.01$ & \\
\hline 1999.41 & $\mathrm{C} 1$ & $0.171 \pm 0.026$ & $0.92 \pm 0.05$ & $-78.9 \pm 0.5$ & $0.43 \pm 0.02$ & \\
\hline 1999.41 & $\mathrm{Ca}$ & $0.140 \pm 0.021$ & $1.50 \pm 0.08$ & $-92.5 \pm 0.5$ & $0.32 \pm 0.02$ & \\
\hline 1999.41 & $\mathrm{C} 2$ & $0.086 \pm 0.013$ & $1.84 \pm 0.09$ & $-96.9 \pm 0.5$ & $0.71 \pm 0.04$ & \\
\hline 1999.41 & $\mathrm{C} 4$ & $0.046 \pm 0.007$ & $3.43 \pm 0.34$ & $-93.9 \pm 1.0$ & $1.74 \pm 0.09$ & \\
\hline 1999.41 & $\mathrm{C} 8$ & $0.025 \pm 0.004$ & $6.47 \pm 0.65$ & $-95.7 \pm 0.9$ & $2.43 \pm 0.24$ & \\
\hline 1999.41 & $\mathrm{C} 12$ & $0.020 \pm 0.003$ & $9.40 \pm 0.90$ & $-95.6 \pm 1.0$ & $2.96 \pm 0.30$ & \\
\hline 1999.41 & $\mathrm{C} 30$ & $0.036 \pm 0.006$ & $27.91 \pm 2.40$ & $-103.1 \pm 3.2$ & $7.51 \pm 0.75$ & \\
\hline 2001.09 & $\mathrm{r}$ & $1.317 \pm 0.146$ & $0.00 \pm 0.01$ & $0.0 \pm 0.5$ & $0.13 \pm 0.09$ & 1.1756 \\
\hline 2001.09 & $\mathrm{C} 0$ & $0.437 \pm 0.068$ & $0.37 \pm 0.07$ & $-81.0 \pm 1.1$ & $0.30 \pm 0.03$ & \\
\hline 2001.09 & $\mathrm{C} 1$ & $0.259 \pm 0.094$ & $0.93 \pm 0.12$ & $-79.4 \pm 1.7$ & $0.42 \pm 0.19$ & \\
\hline 2001.09 & $\mathrm{Ca}$ & $0.179 \pm 0.032$ & $1.47 \pm 0.04$ & $-91.6 \pm 0.8$ & $0.45 \pm 0.11$ & \\
\hline 2001.09 & $\mathrm{C} 2$ & $0.059 \pm 0.008$ & $1.95 \pm 0.04$ & $-93.7 \pm 0.6$ & $0.72 \pm 0.04$ & \\
\hline 2001.09 & $\mathrm{C} 4$ & $0.055 \pm 0.010$ & $4.11 \pm 0.33$ & $-91.0 \pm 0.9$ & $2.44 \pm 0.64$ & \\
\hline 2001.09 & $\mathrm{C} 8$ & $0.027 \pm 0.003$ & $6.12 \pm 0.15$ & $-96.5 \pm 0.3$ & $2.29 \pm 0.13$ & \\
\hline 2001.09 & $\mathrm{C} 12$ & $0.024 \pm 0.008$ & $8.95 \pm 0.73$ & $-94.1 \pm 0.5$ & $2.90 \pm 0.37$ & \\
\hline 2001.09 & $\mathrm{C} 30$ & $0.018 \pm 0.003$ & $27.61 \pm 0.12$ & $-103.8 \pm 0.3$ & $7.18 \pm 0.19$ & \\
\hline & & & $15 \mathrm{GHz}$ & & & \\
\hline 1994.67 & $\mathrm{r}$ & $1.115 \pm 0.167$ & 0.00 & 0.00 & $0.13 \pm 0.01$ & 0.43 \\
\hline 1994.67 & $\mathrm{CO}$ & $0.176 \pm 0.030$ & $0.39 \pm 0.03$ & $-92.8 \pm 3.9$ & $0.40 \pm 0.05$ & \\
\hline 1994.67 & $\mathrm{Ca}$ & $0.097 \pm 0.015$ & $1.46 \pm 0.04$ & $-93.9 \pm 1.5$ & $0.61 \pm 0.05$ & \\
\hline 1994.67 & $\mathrm{C} 4$ & $0.030 \pm 0.010$ & $3.43 \pm 0.10$ & $-90.6 \pm 1.7$ & $1.57 \pm 0.12$ & \\
\hline 1996.38 & $\mathrm{r}$ & $1.559 \pm 0.234$ & 0.00 & 0.00 & $0.12 \pm 0.01$ & 0.43219569 \\
\hline 1996.38 & $\mathrm{CO}$ & $0.572 \pm 0.086$ & $0.25 \pm 0.01$ & $-93.7 \pm 2.1$ & $0.23 \pm 0.01$ & \\
\hline 1996.38 & $\mathrm{C} 1$ & $0.094 \pm 0.014$ & $0.67 \pm 0.03$ & $-98.0 \pm 1.1$ & $0.35 \pm 0.02$ & \\
\hline 1996.38 & $\mathrm{Ca}$ & $0.191 \pm 0.029$ & $1.44 \pm 0.07$ & $-92.9 \pm 0.6$ & $0.45 \pm 0.02$ & \\
\hline 1996.38 & $\mathrm{C} 2$ & $0.124 \pm 0.019$ & $1.74 \pm 0.09$ & $-96.1 \pm 0.5$ & $0.45 \pm 0.02$ & \\
\hline 1996.38 & $\mathrm{C} 4$ & $0.022 \pm 0.003$ & $3.38 \pm 0.34$ & $-86.6 \pm 1.0$ & $1.43 \pm 0.14$ & \\
\hline 1997.20 & $\mathrm{r}$ & $1.362 \pm 0.204$ & 0.00 & 0.00 & $0.11 \pm 0.01$ & 1.4601196 \\
\hline 1997.20 & $\mathrm{C} 0$ & $0.295 \pm 0.044$ & $0.30 \pm 0.02$ & $-89.0 \pm 1.6$ & $0.31 \pm 0.02$ & \\
\hline 1997.20 & $\mathrm{C} 1$ & $0.135 \pm 0.020$ & $0.84 \pm 0.04$ & $-90.3 \pm 0.9$ & $0.55 \pm 0.03$ & \\
\hline 1997.20 & $\mathrm{Ca}$ & $0.111 \pm 0.017$ & $1.47 \pm 0.07$ & $-96.5 \pm 0.5$ & $0.39 \pm 0.02$ & \\
\hline 1997.20 & $\mathrm{C} 2$ & $0.101 \pm 0.015$ & $1.78 \pm 0.09$ & $-96.3 \pm 0.5$ & $0.59 \pm 0.03$ & \\
\hline 1997.20 & $\mathrm{C} 4$ & $0.030 \pm 0.005$ & $3.13 \pm 0.31$ & $-90.9 \pm 1.0$ & $1.64 \pm 0.16$ & \\
\hline 1997.20 & $\mathrm{C} 8$ & $0.034 \pm 0.005$ & $6.81 \pm 0.68$ & $-97.5 \pm 1.0$ & $3.40 \pm 0.34$ & \\
\hline 1998.22 & $\mathrm{r}$ & $1.089 \pm 0.163$ & 0.00 & 0.00 & $0.25 \pm 0.01$ & 0.44147467 \\
\hline 1998.22 & $\mathrm{CO}$ & $0.916 \pm 0.137$ & $0.23 \pm 0.01$ & $-77.2 \pm 5.3$ & $0.30 \pm 0.02$ & \\
\hline 1998.22 & $\mathrm{C} 1$ & $0.119 \pm 0.018$ & $0.93 \pm 0.05$ & $-83.7 \pm 1.2$ & $0.47 \pm 0.02$ & \\
\hline 1998.22 & $\mathrm{Ca}$ & $0.247 \pm 0.037$ & $1.49 \pm 0.07$ & $-95.0 \pm 0.7$ & $0.46 \pm 0.02$ & \\
\hline 1998.22 & $\mathrm{C} 2$ & $0.049 \pm 0.007$ & $2.04 \pm 0.20$ & $-96.8 \pm 1.0$ & $0.62 \pm 0.06$ & \\
\hline 1998.22 & $\mathrm{C} 4$ & $0.038 \pm 0.008$ & $3.40 \pm 0.34$ & $-89.6 \pm 1.4$ & $2.07 \pm 0.21$ & \\
\hline
\end{tabular}


A\&A 511, A57 (2010)

Table 1. continued.

\begin{tabular}{|c|c|c|c|c|c|c|}
\hline (1) & (2) & (3) & (4) & (5) & (6) & (7) \\
\hline Epoch & Id. & S [Jy] & $\mathrm{r}$ [mas] & $\theta[\mathrm{deg}]$ & M.A. [mas] & $\chi^{2}$ \\
\hline 1998.84 & $\mathrm{r}$ & $1.113 \pm 0.167$ & 0.00 & 0.00 & $0.08 \pm 0.01$ & 1.4787256 \\
\hline 1998.84 & $\mathrm{CO}$ & $0.650 \pm 0.098$ & $0.18 \pm 0.02$ & $-86.2 \pm 9.4$ & $0.35 \pm 0.02$ & \\
\hline 1998.84 & $\mathrm{C} 1$ & $0.102 \pm 0.015$ & $0.92 \pm 0.05$ & $-75.6 \pm 2.8$ & $0.60 \pm 0.03$ & \\
\hline 1998.84 & $\mathrm{Ca}$ & $0.141 \pm 0.021$ & $1.42 \pm 0.07$ & $-92.4 \pm 0.6$ & $0.22 \pm 0.01$ & \\
\hline 1998.84 & $\mathrm{C} 2$ & $0.147 \pm 0.022$ & $1.67 \pm 0.08$ & $-99.0 \pm 1.3$ & $0.53 \pm 0.03$ & \\
\hline 1999.57 & $\mathrm{r}$ & $2.015 \pm 0.302$ & 0.00 & 0.0 & $0.12 \pm 0.01$ & 1.1925775 \\
\hline 1999.57 & $\mathrm{CO}$ & $0.430 \pm 0.064$ & $0.33 \pm 0.02$ & $-77.0 \pm 2.1$ & $0.26 \pm 0.01$ & \\
\hline 1999.57 & $\mathrm{C} 1$ & $0.178 \pm 0.027$ & $0.84 \pm 0.04$ & $-77.1 \pm 0.9$ & $0.31 \pm 0.02$ & \\
\hline 1999.57 & $\mathrm{Ca}$ & $0.048 \pm 0.007$ & $1.32 \pm 0.07$ & $-83.5 \pm 0.6$ & $0.01 \pm$ & \\
\hline 1999.57 & $\mathrm{C} 2$ & $0.131 \pm 0.020$ & $1.57 \pm 0.08$ & $-92.5 \pm 0.6$ & $0.42 \pm 0.07$ & \\
\hline 1999.57 & & $0.040 \pm 0.006$ & $1.98 \pm 0.10$ & $-93.4 \pm 1.0$ & $0.68 \pm 0.11$ & \\
\hline 1999.57 & $\mathrm{C} 4$ & $0.032 \pm 0.005$ & $3.28 \pm 0.33$ & $-92.8 \pm 1.1$ & $1.57 \pm 0.14$ & \\
\hline 1999.57 & & $0.007 \pm 0.002$ & $5.14 \pm 0.51$ & $-88.7 \pm 2.0$ & $1.01 \pm 0.18$ & \\
\hline 1999.57 & C8 & $0.020 \pm 0.004$ & $7.38 \pm 0.74$ & $-96.2 \pm 2.0$ & $2.47 \pm 0.34$ & \\
\hline 1999.57 & $\mathrm{C} 12$ & $0.006 \pm 0.001$ & $10.26 \pm 1.16$ & $-91.7 \pm 2.0$ & $3.04 \pm 0.71$ & \\
\hline 1999.85 & $\mathrm{r}$ & $1.603 \pm 0.241$ & 0.00 & $\overline{0.0}$ & $0.090 \pm 0.01$ & 1.1036355 \\
\hline 1999.85 & $\mathrm{C} 0$ & $0.449 \pm 0.067$ & $0.28 \pm 0.05$ & $-79.6 \pm 2.9$ & $0.24 \pm 0.01$ & \\
\hline 1999.85 & $\mathrm{C} 1$ & $0.168 \pm 0.024$ & $0.94 \pm 0.06$ & $-76.2 \pm 1.8$ & $0.39 \pm 0.02$ & \\
\hline 1999.85 & $\mathrm{Ca}$ & $0.093 \pm 0.015$ & $1.38 \pm 0.17$ & $-86.8 \pm 0.6$ & $0.17 \pm 0.01$ & \\
\hline 1999.85 & $\mathrm{C} 2$ & $0.098 \pm 0.015$ & $1.64 \pm 0.10$ & $-94.5 \pm 0.5$ & $0.42 \pm 0.02$ & \\
\hline 1999.85 & $\mathrm{C} 4$ & $0.039 \pm 0.007$ & $2.44 \pm 0.13$ & $-97.5 \pm 2.8$ & $1.27 \pm 0.13$ & \\
\hline 1999.85 & & $0.021 \pm 0.004$ & $4.55 \pm 0.12$ & $-88.1 \pm 1.0$ & $1.61 \pm 0.16$ & \\
\hline 1999.85 & $\mathrm{C} 8$ & $0.013 \pm 0.003$ & $7.86 \pm 0.18$ & $-97.6 \pm 2.0$ & $2.05 \pm 0.21$ & \\
\hline 2000.46 & $\mathrm{r}$ & $1.606 \pm 0.241$ & 0.00 & 0.00 & $0.13 \pm 0.01$ & 0.46644555 \\
\hline 2000.46 & $\mathrm{C} 0$ & $0.364 \pm 0.055$ & $0.35 \pm 0.02$ & $-79.2 \pm 1.8$ & $0.27 \pm 0.01$ & \\
\hline 2000.46 & $\mathrm{C} 1$ & $0.144 \pm 0.022$ & $0.96 \pm 0.05$ & $-79.3 \pm 0.7$ & $0.36 \pm 0.02$ & \\
\hline 2000.46 & $\mathrm{Ca}$ & $0.134 \pm 0.020$ & $1.44 \pm 0.07$ & $-88.8 \pm 0.5$ & $0.29 \pm 0.02$ & \\
\hline 2000.46 & $\mathrm{C} 2$ & $0.105 \pm 0.016$ & $1.70 \pm 0.03$ & $-92.1 \pm 0.7$ & $0.62 \pm 0.03$ & \\
\hline 2000.46 & $\mathrm{C} 4$ & $0.055 \pm 0.008$ & $3.61 \pm 0.72$ & $-91.9 \pm 1.1$ & $1.95 \pm 0.20$ & \\
\hline 2002.13 & $\mathrm{r}$ & $1.702 \pm 0.251$ & $0.00 \pm 0.01$ & $0.0 \pm 0.3$ & $0.11 \pm 0.02$ & 1.3686 \\
\hline 2002.13 & $\mathrm{C} 0$ & $0.151 \pm 0.023$ & $0.37 \pm 0.01$ & $-94.6 \pm 1.2$ & $0.29 \pm 0.01$ & \\
\hline 2002.13 & B3 & $0.008 \pm 0.001$ & $0.76 \pm 0.02$ & $-89.8 \pm 1.2$ & $0.14 \pm 0.03$ & \\
\hline 2002.13 & $\mathrm{C} 1$ & $0.072 \pm 0.012$ & $1.02 \pm 0.02$ & $-75.2 \pm 0.5$ & $0.35 \pm 0.02$ & \\
\hline 2002.13 & $\mathrm{Ca}$ & $0.318 \pm 0.043$ & $1.43 \pm 0.02$ & $-88.1 \pm 0.5$ & $0.48 \pm 0.05$ & \\
\hline 2002.13 & $\mathrm{C} 2$ & $0.029 \pm 0.004$ & $2.02 \pm 0.02$ & $-94.0 \pm 0.5$ & $0.68 \pm 0.01$ & \\
\hline 2002.13 & $\mathrm{C} 4$ & $0.031 \pm 0.005$ & $3.61 \pm 0.05$ & $-89.0 \pm 0.8$ & $1.94 \pm 0.02$ & \\
\hline 2002.13 & $\mathrm{C} 8$ & $0.044 \pm 0.007$ & $6.37 \pm 0.09$ & $-95.1 \pm 0.8$ & $3.40 \pm 0.02$ & \\
\hline 2002.13 & $\mathrm{C} 12$ & $0.009 \pm 0.001$ & $11.36 \pm 0.37$ & $-94.6 \pm 1.9$ & $3.37 \pm 0.67$ & \\
\hline 2003.10 & $\mathrm{r}$ & $1.245 \pm 0.179$ & $0.00 \pm 0.02$ & $0.0 \pm 0.2$ & $0.10 \pm 0.03$ & 1.5375 \\
\hline 2003.10 & $\mathrm{CO}$ & $0.310 \pm 0.048$ & $0.31 \pm 0.03$ & $-76.9 \pm 1.7$ & $0.27 \pm 0.01$ & \\
\hline 2003.10 & $\mathrm{C} 1$ & $0.039 \pm 0.008$ & $0.66 \pm 0.03$ & $-84.2 \pm 1.3$ & $0.09 \pm 0.04$ & \\
\hline 2003.10 & $\mathrm{Ca}$ & $0.287 \pm 0.058$ & $1.42 \pm 0.01$ & $-88.4 \pm 0.3$ & $0.35 \pm 0.09$ & \\
\hline 2003.10 & $\mathrm{C} 2$ & $0.113 \pm 0.045$ & $1.78 \pm 0.24$ & $-89.1 \pm 0.8$ & $1.00 \pm 0.26$ & \\
\hline 2003.10 & B3 & $0.035 \pm 0.016$ & $2.64 \pm 0.38$ & $-88.9 \pm 1.2$ & $1.17 \pm 0.19$ & \\
\hline 2003.10 & $\mathrm{C} 4$ & $0.039 \pm 0.006$ & $4.67 \pm 0.32$ & $-90.0 \pm 0.8$ & $2.01 \pm 0.35$ & \\
\hline 2003.10 & $\mathrm{C} 8$ & $0.031 \pm 0.004$ & $7.44 \pm 0.49$ & $-97.1 \pm 0.7$ & $3.00 \pm 0.16$ & \\
\hline 2003.10 & $\mathrm{C} 12$ & $0.015 \pm 0.004$ & $12.37 \pm 0.78$ & $-93.2 \pm 0.6$ & $4.31 \pm 0.64$ & \\
\hline 2004.27 & $\mathrm{r}$ & $1.264 \pm 0.185$ & $0.00 \pm 0.01$ & $0.0 \pm 0.1$ & $0.09 \pm 0.03$ & 1.4844 \\
\hline 2004.27 & $\mathrm{C} 0$ & $0.117 \pm 0.017$ & $0.33 \pm 0.01$ & $-89.1 \pm 1.3$ & $0.25 \pm 0.01$ & \\
\hline 2004.27 & $\mathrm{C} 1$ & $0.124 \pm 0.018$ & $1.00 \pm 0.05$ & $-78.0 \pm 0.6$ & $0.38 \pm 0.05$ & \\
\hline 2004.27 & $\mathrm{Ca}$ & $0.185 \pm 0.028$ & $1.47 \pm 0.02$ & $-90.6 \pm 0.4$ & $0.31 \pm 0.06$ & \\
\hline 2004.27 & $\mathrm{C} 2$ & $0.046 \pm 0.009$ & $1.76 \pm 0.06$ & $-95.6 \pm 0.5$ & $0.49 \pm 0.04$ & \\
\hline 2004.27 & $\mathrm{C} 4$ & $0.044 \pm 0.007$ & $3.64 \pm 0.09$ & $-86.9 \pm 0.9$ & $1.83 \pm 0.05$ & \\
\hline 2004.27 & $\mathrm{C} 8$ & $0.046 \pm 0.007$ & $6.42 \pm 0.24$ & $-96.3 \pm 0.8$ & $2.71 \pm 0.08$ & \\
\hline 2004.27 & C12 & $0.014 \pm 0.003$ & $9.81 \pm 0.30$ & $-96.3 \pm 0.6$ & $3.09 \pm 0.55$ & \\
\hline 2005.30 & $\mathrm{r}$ & $0.819 \pm 0.119$ & $0.00 \pm 0.01$ & $0.0 \pm 0.2$ & $0.12 \pm 0.04$ & 0.9761 \\
\hline 2005.30 & $\mathrm{C} 0$ & $0.160 \pm 0.028$ & $0.22 \pm 0.02$ & $-61.6 \pm 0.3$ & $0.05 \pm 0.03$ & \\
\hline 2005.30 & $\mathrm{C} 1$ & $0.091 \pm 0.014$ & $0.61 \pm 0.02$ & $-75.8 \pm 1.3$ & $0.35 \pm 0.02$ & \\
\hline 2005.30 & $\mathrm{Ca}$ & $0.297 \pm 0.045$ & $1.47 \pm 0.01$ & $-87.4 \pm 0.6$ & $0.42 \pm 0.03$ & \\
\hline 2005.30 & $\mathrm{C} 2$ & $0.040 \pm 0.011$ & $1.98 \pm 0.06$ & $-84.1 \pm 0.5$ & $0.51 \pm 0.07$ & \\
\hline 2005.30 & $\mathrm{C} 4$ & $0.018 \pm 0.005$ & $3.98 \pm 0.09$ & $-84.9 \pm 0.8$ & $1.12 \pm 0.34$ & \\
\hline 2005.30 & $\mathrm{C} 8$ & $0.064 \pm 0.009$ & $6.08 \pm 0.30$ & $-94.7 \pm 1.0$ & $3.04 \pm 0.23$ & \\
\hline 2005.30 & $\mathrm{C} 12$ & $0.010 \pm 0.005$ & $9.80 \pm 0.97$ & $-91.9 \pm 2.5$ & $2.90 \pm 0.70$ & \\
\hline
\end{tabular}


Table 1. continued.

\begin{tabular}{|c|c|c|c|c|c|c|}
\hline (1) & (2) & (3) & (4) & (5) & (6) & (7) \\
\hline Epoch & Id. & $\mathrm{S}[\mathrm{Jy}]$ & $\mathrm{r}$ [mas] & $\theta[\mathrm{deg}]$ & M.A. [mas] & $\chi^{2}$ \\
\hline 2005.68 & $\mathrm{r}$ & $1.381 \pm 0.203$ & $0.00 \pm 0.01$ & $0.0 \pm 0.3$ & $0.09 \pm 0.02$ & 0.8685 \\
\hline 2005.68 & $\mathrm{C} 0$ & $0.146 \pm 0.022$ & $0.25 \pm 0.03$ & $-74.2 \pm 1.5$ & $0.21 \pm 0.02$ & \\
\hline 2005.68 & $\mathrm{C} 1$ & $0.048 \pm 0.008$ & $0.75 \pm 0.02$ & $-78.0 \pm 0.8$ & $0.32 \pm 0.03$ & \\
\hline 2005.68 & $\mathrm{Ca}$ & $0.244 \pm 0.064$ & $1.44 \pm 0.02$ & $-89.0 \pm 0.3$ & $0.31 \pm 0.09$ & \\
\hline 2005.68 & $\mathrm{C} 2$ & $0.120 \pm 0.019$ & $1.71 \pm 0.04$ & $-88.8 \pm 0.5$ & $0.55 \pm 0.02$ & \\
\hline 2005.68 & $\mathrm{C} 4$ & $0.021 \pm 0.007$ & $3.44 \pm 0.29$ & $-87.0 \pm 1.3$ & $1.50 \pm 0.20$ & \\
\hline 2005.68 & B3 & $0.013 \pm 0.001$ & $4.72 \pm 0.06$ & $-83.7 \pm 0.4$ & $1.16 \pm 0.03$ & \\
\hline 2005.68 & $\mathrm{C} 8$ & $0.058 \pm 0.006$ & $6.37 \pm 0.11$ & $-97.4 \pm 0.6$ & $2.49 \pm 0.18$ & \\
\hline 2005.68 & $\mathrm{C} 12$ & $0.022 \pm 0.001$ & $8.98 \pm 0.20$ & $-93.7 \pm 0.7$ & $3.68 \pm 0.22$ & \\
\hline
\end{tabular}

Table 2. Gaussian model-fit results for S5 1803+784 obtained from the literature. The columns list the epoch of observation, the jet-component identification, the flux density, the radial distance of the component center from the core, the position angle of the center of the component, the FWHM major axis, and for elliptical Gaussian fits, the axial ratio and the position angle of the major axis. The final column provides a reference to the original publication.

\begin{tabular}{|c|c|c|c|c|c|c|c|c|}
\hline $\begin{array}{c}(1) \\
\text { Epoch }\end{array}$ & $\begin{array}{l}\text { (2) } \\
\text { Id. }\end{array}$ & $\begin{array}{r}(3) \\
\mathrm{S}[\mathrm{Jy}]\end{array}$ & $\begin{array}{r}(4) \\
\mathrm{r}[\mathrm{mas}]\end{array}$ & $\begin{array}{r}(5) \\
\theta[\mathrm{deg}]\end{array}$ & $\begin{array}{r}(6) \\
\text { Ma.A. [mas] }\end{array}$ & $\begin{array}{r}(7) \\
\text { Axial ratio }\end{array}$ & $\begin{array}{r}(8) \\
\Phi[\mathrm{deg}]\end{array}$ & $\begin{array}{c}(9) \\
\text { Reference }\end{array}$ \\
\hline & & & & $2.32 \mathrm{GHz}$ & & & & \\
\hline 1994.52 & 1 & 1.63 & 0.00 & 0.00 & 1.37 & 0.00 & 87 & Fey et al. (1996) \\
\hline 1994.52 & 2 & 0.34 & 4.0 & -94 & 7.24 & 0.28 & 81 & \\
\hline 1994.52 & 3 & 0.28 & 27.6 & -105 & 12.11 & 1.00 & & \\
\hline \multirow[b]{2}{*}{1997.79} & & & & $5 \mathrm{GHz}$ & & & & \\
\hline & $\mathrm{C} 1$ & 0.413 & 1.38 & -98 & 0.65 & 0.82 & 50 & Lister et al. (2001) \\
\hline & & & & $8 \mathrm{GHz}$ & & & & \\
\hline 1988.79 & Core & 2.45 & 0.00 & 0.0 & 0.1 & & & Tateyama et al. (2002) \\
\hline 1988.79 & $\mathrm{C} 1$ & 0.23 & 1.50 & -95 & 0.1 & & & \\
\hline 1988.79 & $\mathrm{C} 2$ & 0.21 & 0.90 & -95 & 0.1 & & & \\
\hline 1989.96 & Core & 2.77 & 0.00 & 0.0 & 0.1 & & & Tateyama et al. (2002) \\
\hline 1989.96 & $\mathrm{C} 1$ & 0.22 & 1.51 & -94 & 0.1 & & & \\
\hline 1989.96 & $\mathrm{C} 2$ & 0.20 & 1.09 & -90 & 0.3 & & & \\
\hline 1989.96 & $\mathrm{E}$ & 0.65 & 0.40 & -81 & 0.2 & & & \\
\hline 1991.96 & Core & 1.38 & 0.00 & 0.0 & 0.1 & & & Tateyama et al. (2002) \\
\hline 1991.96 & $\mathrm{C} 1$ & 0.14 & 1.71 & -90.9 & 0.1 & & & \\
\hline 1991.96 & $\mathrm{C} 2$ & 0.27 & 1.28 & -87.4 & 0.1 & & & \\
\hline 1991.96 & $\mathrm{E}$ & 0.06 & 0.58 & -85.1 & 0.1 & & & \\
\hline 1992.62 & Core & 1.68 & 0.00 & 0.0 & 0.2 & & & Tateyama et al. (2002) \\
\hline 1992.62 & $\mathrm{C} 1$ & 0.11 & 1.80 & -90 & 0.1 & & & \\
\hline 1992.62 & $\mathrm{C} 2$ & 0.40 & 1.27 & -95 & 0.1 & & & \\
\hline 1992.62 & $\mathrm{E}$ & 0.32 & 0.48 & -93 & 0.01 & & & \\
\hline 1993.87 & Core & 1.25 & 0.00 & 0.0 & 0.2 & & & Tateyama et al. (2002) \\
\hline 1993.87 & $\mathrm{C} 2$ & 0.33 & 1.39 & -97.2 & 0.1 & & & \\
\hline 1994.71 & Core & 1.49 & 0.00 & 0.0 & 0.2 & & & Tateyama et al. (2002) \\
\hline 1994.71 & $\mathrm{C} 2$ & 0.17 & 1.43 & -96.2 & 0.1 & & & \\
\hline 1995.71 & Core & 2.10 & 0.00 & 0.0 & 0.25 & & & Tateyama et al. (2002) \\
\hline 1995.71 & $\mathrm{C} 2$ & 0.28 & 1.52 & -93.0 & 0.1 & & & \\
\hline 1995.71 & $\mathrm{C} 3$ & 0.18 & 1.12 & -93.1 & 0.1 & & & \\
\hline 1996.29 & Core & 1.57 & 0.00 & 0.0 & 0.2 & & & Tateyama et al. (2002) \\
\hline 1996.29 & $\mathrm{C} 2$ & 0.10 & 1.59 & -91 & 0.1 & & & \\
\hline 1996.29 & $\mathrm{C} 3$ & 0.04 & 1.10 & -97 & 0.1 & & & \\
\hline 1996.29 & $\mathrm{E}$ & 0.18 & 0.44 & -101 & 0.1 & & & \\
\hline 1996.71 & Core & 1.56 & 0.00 & 0.0 & 0.2 & & & Tateyama et al. (2002) \\
\hline 1996.71 & $\mathrm{C} 2$ & 0.15 & 1.73 & -92.4 & 0.1 & & & \\
\hline 1996.71 & $\mathrm{C} 3$ & 0.10 & 1.25 & -96.1 & 0.1 & & & \\
\hline 1996.71 & $\mathrm{E}$ & 0.17 & 0.51 & -89.2 & 0.1 & & & \\
\hline 1998.79 & Core & 1.60 & 0.00 & 0.0 & 0.2 & & & Tateyama et al. (2002) \\
\hline 1998.79 & $\mathrm{C} 2$ & 0.13 & 1.82 & -97.0 & 0.1 & & & \\
\hline 1998.79 & $\mathrm{C} 3$ & 0.26 & 1.30 & -93.7 & 0.1 & & & \\
\hline 1998.79 & $\mathrm{E}$ & 0.41 & 0.40 & -93.3 & 0.1 & & & \\
\hline 1999.62 & Core & 1.89 & 0.00 & 0.0 & 0.2 & & & Tateyama et al. (2002) \\
\hline 1999.62 & $\mathrm{C} 2$ & 0.09 & 1.86 & -90.0 & 0.1 & & & \\
\hline 1999.62 & C3 & 0.19 & 1.33 & -88.1 & 0.1 & & & \\
\hline 1999.62 & $\mathrm{E}$ & 0.28 & 0.62 & -77.8 & 0.1 & & & \\
\hline
\end{tabular}


Table 2. continued.

\begin{tabular}{|c|c|c|c|c|c|c|c|c|}
\hline $\begin{array}{c}(1) \\
\text { Epoch }\end{array}$ & $\begin{array}{l}(2) \\
\text { Id. }\end{array}$ & $\begin{array}{r}(3) \\
\text { S [Jy] }\end{array}$ & $\begin{array}{r}(4) \\
\mathrm{r}[\mathrm{mas}]\end{array}$ & $\begin{array}{r}(5) \\
\theta[\mathrm{deg}]\end{array}$ & $\begin{array}{r}(6) \\
\text { Ma.A. [mas] }\end{array}$ & $\begin{array}{r}(7) \\
\text { Axial ratio }\end{array}$ & $\begin{array}{r}(8) \\
\Phi[\mathrm{deg}]\end{array}$ & $\begin{array}{c}(9) \\
\text { Reference }\end{array}$ \\
\hline & & & & $8.4 \mathrm{GHz}$ & & & & \multirow{9}{*}{ Ros et al. (2001) } \\
\hline 1997.93 & XA & $1.493 \pm 0.001$ & 0.00 & & $0.2 \pm 0.1$ & $0.8 \pm 0.1$ & $+119 \pm 1$ & \\
\hline 1997.93 & $\mathrm{XB}$ & $0.231 \pm 0.001$ & $0.6 \pm 0.2$ & $-86 \pm 19$ & $0.7 \pm 0.1$ & $0.6 \pm 0.1$ & $+102 \pm 1$ & \\
\hline 1997.93 & $\mathrm{XC}$ & $0.274 \pm 0.001$ & $1.4 \pm 0.2$ & $-97 \pm 8$ & $0.6 \pm 0.1$ & $0.7 \pm 0.1$ & $+41 \pm 1$ & \\
\hline 1997.93 & $\mathrm{XD}$ & $0.036 \pm 0.001$ & $2.0 \pm 0.2$ & $-98 \pm 5$ & $0.5 \pm 0.1$ & $0.2 \pm 0.1$ & $-12 \pm 4$ & \\
\hline 1997.93 & $\mathrm{XE}$ & $0.024 \pm 0.001$ & $2.7 \pm 0.2$ & $-97 \pm 4$ & $1.4 \pm 0.1$ & $0.4 \pm 0.1$ & $+1 \pm 3$ & \\
\hline 1997.93 & $\mathrm{XF}$ & $0.018 \pm 0.001$ & $3.9 \pm 0.2$ & $-89 \pm 3$ & $1.1 \pm 0.1$ & $0.9 \pm 0.1$ & $-30 \pm 16$ & \\
\hline 1997.93 & XG & $0.019 \pm 0.001$ & $6.3 \pm 0.2$ & $-96 \pm 3$ & $2.8 \pm 0.2$ & $0.5 \pm 0.1$ & $+31 \pm 4$ & \\
\hline 1997.93 & $\mathrm{XH}$ & $0.025 \pm 0.001$ & $8.9 \pm 0.2$ & $-95 \pm 1$ & $4.1 \pm 0.2$ & $0.5 \pm 0.1$ & $+99 \pm 4$ & \\
\hline 1999.41 & $\mathrm{XA}$ & $1.517 \pm 0.001$ & 0.00 & & $0.2 \pm 0.1$ & $0.6 \pm 0.1$ & $+107 \pm 1$ & \multirow{8}{*}{ Ros et al. (2001) } \\
\hline 1999.41 & $\mathrm{XB}$ & $0.374 \pm 0.001$ & $0.5 \pm 0.2$ & $-81 \pm 21$ & $0.5 \pm 0.1$ & $0.7 \pm 0.1$ & $+117 \pm 1$ & \\
\hline 1999.41 & $\mathrm{XC}$ & $0.192 \pm 0.001$ & $1.4 \pm 0.2$ & $-93 \pm 8$ & $0.5 \pm 0.1$ & $0.3 \pm 0.1$ & $+22 \pm 1$ & \\
\hline 1999.41 & $\mathrm{XD}$ & $0.058 \pm 0.001$ & $1.9 \pm 0.2$ & $-94 \pm 6$ & $0.6 \pm 0.1$ & $0.2 \pm 0.1$ & $+3 \pm 2$ & \\
\hline 1999.41 & $\mathrm{XE}$ & $0.030 \pm 0.001$ & $2.7 \pm 0.2$ & $-93 \pm 4$ & $1.9 \pm 0.1$ & $0.4 \pm 0.1$ & $+126 \pm 2$ & \\
\hline 1999.41 & $\mathrm{XF}$ & $0.030 \pm 0.001$ & $4.0 \pm 0.2$ & $-97 \pm 3$ & $2.4 \pm 0.1$ & $0.4 \pm 0.1$ & $+111 \pm 1$ & \\
\hline 1999.41 & XG & $0.020 \pm 0.001$ & $7.0 \pm 0.2$ & $-97 \pm 2$ & $2.9 \pm 0.2$ & $0.6 \pm 0.1$ & $+115 \pm 5$ & \\
\hline 1999.41 & $\mathrm{XH}$ & $0.017 \pm 0.001$ & $9.4 \pm 0.2$ & $-97 \pm 1$ & $3.6 \pm 0.3$ & $0.6 \pm 0.1$ & $+84 \pm 5$ & \\
\hline & & & & $8.55 \mathrm{GHz}$ & & & & \multirow{5}{*}{ Fey et al. (1996) } \\
\hline 1994.52 & 1 & 1.91 & 0.00 & & 0.19 & 0.63 & -81 & \\
\hline 1994.52 & 2 & 0.38 & 1.0 & -92 & 1.53 & 0.28 & 83 & \\
\hline 1994.52 & 3 & 0.05 & 3.5 & -86 & 1.26 & 1.00 & & \\
\hline 1994.52 & 4 & 0.05 & 6.6 & -95 & 2.57 & 1.00 & & \\
\hline & & & & $86 \mathrm{GHz}$ & & & & \multirow{3}{*}{ Lobanov et al. (2000) } \\
\hline 1993.29 & $\mathrm{C}$ & $0.448 \pm 0.063$ & 0.00 & & $0.08 \pm 0.03$ & & & \\
\hline 1993.29 & $\mathrm{~J} 1$ & $0.376 \pm 0.056$ & $0.27 \pm 0.06$ & $-0.9 \pm 8.0$ & $0.08 \pm 0.03$ & & & \\
\hline
\end{tabular}

Table 7. Discrete cross-correlations (based on components from the $8 \mathrm{GHz}$ model fits) and Pearson's correlation coefficients (from $15 \mathrm{GHz}$ model fits), calculated between the time series of various pairs of jet parameters and/or the total flux densities. Column 1 gives the frequency of the analyzed data set; Col. 2 lists the individual jet component from which the parameters to correlate are taken; Cols. 3 and 4 list the parameters correlated. For $8 \mathrm{GHz}$ data, the DCF peak and $\tau$ are the peak of discrete cross-correlation function and the associated time lag in years. For $15 \mathrm{GHz}$ data, Corr.coeff. is the value of the Pearson's correlation coefficient, and $p$ is the probability of getting such a high correlation by chance.

\begin{tabular}{|c|c|c|c|c|c|c|c|}
\hline $\begin{array}{l}v \\
{[\mathrm{GHz}]}\end{array}$ & $\begin{array}{l}\text { Comp. } \\
\text { ID }\end{array}$ & Par. 1 & Par. 2 & $\begin{array}{l}\text { DCF } \\
\text { peak }\end{array}$ & $\tau$ & $\begin{array}{l}\text { Corr. } \\
\text { coeff. }\end{array}$ & $p$ \\
\hline \multirow[t]{6}{*}{8} & $\mathrm{Ca}$ & core sep. & total flux $8 \mathrm{GHz}$ & $-0.68 \pm 0.06$ & $-1.12 \pm 0.05$ & & \\
\hline & & p.a. & total flux $8 \mathrm{GHz}$ & $-0.77 \pm 0.04$ & $0.72 \pm 0.06$ & & \\
\hline & $\mathrm{C} 2_{1}$ & core sep. & total flux $8 \mathrm{GHz}$ & $0.56 \pm 0.06$ & $-0.07 \pm 0.08$ & & \\
\hline & $\mathrm{C} 2_{3}$ & p.a. & total flux $8 \mathrm{GHz}$ & $0.60 \pm 0.07$ & $-0.15 \pm 0.08$ & & \\
\hline & $\mathrm{C} 1$ & core sep. & total flux $14.5 \mathrm{GHz}$ & $0.41 \pm 0.07$ & $-0.07 \pm 0.04$ & & \\
\hline & & p.a. & total flux $14.5 \mathrm{GHz}$ & $0.61 \pm 0.05$ & $0.06 \pm 0.06$ & & \\
\hline \multirow[t]{9}{*}{8} & $\mathrm{C} 1$ & p.a. & core sep. & $0.54 \pm 0.09$ & $0.20 \pm 0.05$ & & \\
\hline & & p.a. & flux & $0.53 \pm 0.08$ & $-0.08 \pm 0.04$ & & \\
\hline & & core sep. & flux & $0.41 \pm 0.06$ & $-0.58 \pm 0.06$ & & \\
\hline & $\mathrm{Ca}$ & p.a. & core sep. & $-0.59 \pm 0.06$ & $-0.59 \pm 0.05$ & & \\
\hline & & p.a. & flux & $0.42 \pm 0.06$ & $-0.56 \pm 0.06$ & & \\
\hline & & core sep. & flux & $-0.64 \pm 0.06$ & $-0.03 \pm 0.04$ & & \\
\hline & $\mathrm{C} 2_{1}$ & p.a. & core sep. & $-0.53 \pm 0.09$ & $0.31 \pm 0.08$ & & \\
\hline & $\mathrm{C} 2_{2}$ & p.a. & core sep. & $-0.61 \pm 0.08$ & $-0.42 \pm 0.04$ & & \\
\hline & $\mathrm{C} 4$ & p.a. & core sep. & $0.97 \pm 0.10$ & $0.09 \pm 0.03$ & & \\
\hline \multirow[t]{9}{*}{15} & $\mathrm{C} 0$ & p.a. & core sep. & & & $-0.44 \pm 0.09$ & 0.131 \\
\hline & & core sep. & flux & & & $-0.52 \pm 0.08$ & 0.067 \\
\hline & $\mathrm{C} 1$ & p.a. & core sep. & & & $0.40 \pm 0.10$ & 0.198 \\
\hline & & core sep. & flux & & & $0.46 \pm 0.09$ & 0.164 \\
\hline & $\mathrm{Ca}$ & p.a. & core sep. & & & $-0.73 \pm 0.05$ & 0.008 \\
\hline & & core sep. & flux & & & $0.57 \pm 0.07$ & 0.054 \\
\hline & $\mathrm{C} 2$ & core sep. & flux & & & $-0.83 \pm 0.03$ & 0.002 \\
\hline & $\mathrm{C} 4$ & p.a. & core sep. & & & $0.58 \pm 0.07$ & 0.064 \\
\hline & & p.a. & flux & & & $-0.51 \pm 0.08$ & 0.107 \\
\hline
\end{tabular}


Table 8. Discrete cross-correlations (based on components from the $8 \mathrm{GHz}$ model fits) and Pearson's correlation coefficients (from $15 \mathrm{GHz}$ model fits), calculated for various pairs jet parameters and the core flux density. Column 1 gives the frequency of the analyzed data set; Cols. 2 and 3 lists the two jet components participating in the correlation; Col. 4 shows the circular Gaussian parameter correlated. When "core" is the first component, its parameter is always flux-density. For $8 \mathrm{GHz}$ data, DCF peak and $\tau$ are the peak of the discrete correlation function and the associated time lag in years. For $15 \mathrm{GHz}$ data, Corr.coeff. is the value of the Pearson's correlation coefficient, and $p$ the probability of getting such a high correlation by chance.

\begin{tabular}{|c|c|c|c|c|c|c|c|}
\hline $\begin{array}{l}v \\
{[\mathrm{GHz}]}\end{array}$ & $\begin{array}{l}\text { Comp. } \\
\text { ID } 1\end{array}$ & $\begin{array}{l}\text { Comp. } \\
\text { ID } 2\end{array}$ & Par. & $\begin{array}{l}\text { DCF } \\
\text { peak }\end{array}$ & $\tau$ & $\begin{array}{l}\text { Corr. } \\
\text { coeff. }\end{array}$ & $p$ \\
\hline \multirow[t]{18}{*}{8} & $\mathrm{C} 0$ & $\mathrm{Ca}$ & core sep. & $-0.48 \pm 0.12$ & $0.54 \pm 0.07$ & & \\
\hline & $\mathrm{C} 0$ & $\mathrm{C} 2_{1}$ & p.a. & $-0.49 \pm 0.14$ & $-0.32 \pm 0.06$ & & \\
\hline & $\mathrm{C} 0$ & $\mathrm{C} 2_{3}$ & p.a. & $0.69 \pm 0.18$ & $0.14 \pm 0.08$ & & \\
\hline & $\mathrm{C} 1$ & $\mathrm{Ca}$ & core sep. & $0.47 \pm 0.06$ & $0.08 \pm 0.06$ & & \\
\hline & & & p.a. & $-0.48 \pm 0.07$ & $0.48 \pm 0.07$ & & \\
\hline & $\mathrm{C} 1$ & $\mathrm{C} 2_{1}$ & core sep. & $-0.59 \pm 0.09$ & $-0.80 \pm 0.09$ & & \\
\hline & & & p.a. & $-0.45 \pm 0.09$ & $-0.29 \pm 0.09$ & & \\
\hline & $\mathrm{C} 1$ & $\mathrm{C} 2_{2}$ & core sep. & $0.53 \pm 0.14$ & $-0.02 \pm 0.06$ & & \\
\hline & & & p.a. & $0.96 \pm 0.03$ & $-0.02 \pm 0.02$ & & \\
\hline & $\mathrm{C} 1$ & $\mathrm{C} 2_{3}$ & core sep. & $0.83 \pm 0.06$ & $0.81 \pm 0.02$ & & \\
\hline & & & p.a. & $0.72 \pm 0.09$ & $-0.06 \pm 0.06$ & & \\
\hline & $\mathrm{Ca}$ & $\mathrm{C} 2_{1}$ & core sep. & $-0.48 \pm 0.09$ & $-0.09 \pm 0.06$ & & \\
\hline & $\mathrm{Ca}$ & $\mathrm{C} 2_{3}$ & core sep. & $0.62 \pm 0.09$ & $0.55 \pm 0.06$ & & \\
\hline & & & p.a. & $-0.61 \pm 0.07$ & $0.49 \pm 0.08$ & & \\
\hline & $\mathrm{C} 2_{1}$ & $\mathrm{C} 2_{2}$ & p.a. & $-0.53 \pm 0.08$ & $0.42 \pm 0.05$ & & \\
\hline & $\mathrm{C} 2_{1}$ & $\mathrm{C} 2_{3}$ & core sep. & $0.62 \pm 0.09$ & $-0.42 \pm 0.06$ & & \\
\hline & & & p.a. & $-0.78 \pm 0.09$ & $0.17 \pm 0.02$ & & \\
\hline & $\mathrm{C} 2_{2}$ & $\mathrm{C} 2_{3}$ & p.a. & $0.79 \pm 0.05$ & $-0.21 \pm 0.05$ & & \\
\hline \multirow[t]{11}{*}{8} & Core & $\mathrm{CO}$ & core sep. & $0.62 \pm 0.09$ & $-0.23 \pm 0.07$ & & \\
\hline & & & p.a. & $0.77 \pm 0.10$ & $-0.09 \pm 0.05$ & & \\
\hline & Core & $\mathrm{C} 1$ & core sep. & $-0.47 \pm 0.07$ & $0.35 \pm 0.08$ & & \\
\hline & & & p.a. & $-0.45 \pm 0.07$ & $-0.31 \pm 0.04$ & & \\
\hline & Core & $\mathrm{Ca}$ & flux & $0.59 \pm 0.06$ & $0.49 \pm 0.10$ & & \\
\hline & & & core sep. & $-0.91 \pm 0.04$ & $0.23 \pm 0.03$ & & \\
\hline & & & p.a. & $-0.83 \pm 0.04$ & $-0.92 \pm 0.04$ & & \\
\hline & Core & $\mathrm{C} 2_{1}$ & core sep. & $0.64 \pm 0.07$ & $0.02 \pm 0.05$ & & \\
\hline & Core & $\mathrm{C} 2_{2}$ & p.a. & $-0.47 \pm 0.08$ & $-0.29 \pm 0.05$ & & \\
\hline & Core & $\mathrm{C} 2_{3}$ & core sep. & $0.77 \pm 0.05$ & $-0.36 \pm 0.04$ & & \\
\hline & & & p.a. & $0.54 \pm 0.08$ & $0.37 \pm 0.10$ & & \\
\hline \multirow[t]{5}{*}{15} & Core & $\mathrm{C} 0$ & core sep. & & & $0.65 \pm 0.05$ & 0.021 \\
\hline & Core & $\mathrm{Ca}$ & core sep. & & & $-0.72 \pm 0.05$ & 0.008 \\
\hline & & & p.a. & & & $0.49 \pm 0.09$ & 0.146 \\
\hline & Core & $\mathrm{C} 2$ & core sep. & & & $-0.49 \pm 0.08$ & 0.124 \\
\hline & Core & $\mathrm{C} 4$ & p.a. & & & $-0.56 \pm 0.08$ & 0.074 \\
\hline \multirow[t]{6}{*}{15} & $\mathrm{C} 0$ & $\mathrm{C} 2$ & p.a. & & & $0.77 \pm 0.04$ & 0.006 \\
\hline & $\mathrm{C} 1$ & $\mathrm{Ca}$ & flux & & & $-0.84 \pm 0.03$ & 0.001 \\
\hline & & & p.a. & & & $-0.67 \pm 0.06$ & 0.025 \\
\hline & $\mathrm{Ca}$ & $\mathrm{C} 2$ & flux & & & $-0.60 \pm 0.07$ & 0.051 \\
\hline & & & core sep. & & & $0.69 \pm 0.06$ & 0.019 \\
\hline & & & p.a. & & & $0.54 \pm 0.08$ & 0.084 \\
\hline
\end{tabular}

